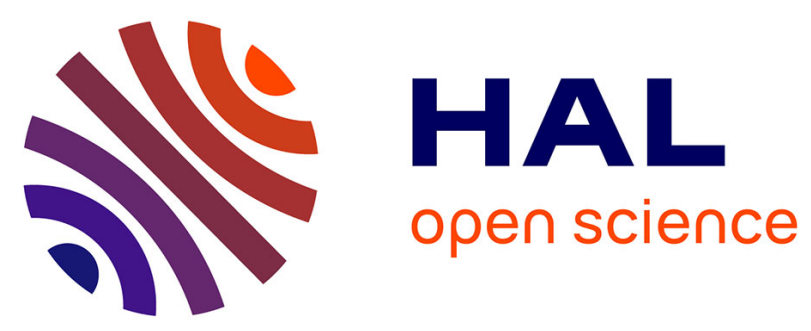

\title{
Stability of discontinuous diffusion coefficients and initial conditions in an inverse problem for the heat equation
}

\author{
Assia Benabdallah, Patricia Gaitan, Jérôme Le Rousseau
}

\section{To cite this version:}

Assia Benabdallah, Patricia Gaitan, Jérôme Le Rousseau. Stability of discontinuous diffusion coefficients and initial conditions in an inverse problem for the heat equation. SIAM Journal on Control and Optimization, 2007, 46 (5), pp.1849-1881. 10.1137/050640047 . hal-00016490v3

\section{HAL Id: hal-00016490 \\ https://hal.science/hal-00016490v3}

Submitted on 20 Oct 2006

HAL is a multi-disciplinary open access archive for the deposit and dissemination of scientific research documents, whether they are published or not. The documents may come from teaching and research institutions in France or abroad, or from public or private research centers.
L'archive ouverte pluridisciplinaire HAL, est destinée au dépôt et à la diffusion de documents scientifiques de niveau recherche, publiés ou non, émanant des établissements d'enseignement et de recherche français ou étrangers, des laboratoires publics ou privés. 


\title{
Stability of discontinuous diffusion coefficients and initial conditions in an inverse problem for the heat equation
}

\author{
Assia Benabdallah, ${ }^{1, a}$, Patricia Gaitan ${ }^{2, b}$, \\ and \\ Jérôme Le Rousseau ${ }^{1, c}$ \\ Laboratoire d'analyse, topologie, probabilités \\ CNRS UMR 6632, Marseille, France.
}

October 20, 2006

\begin{abstract}
We consider the heat equation with a discontinuous diffusion coefficient and give uniqueness and stability results for both the diffusion coefficient and the initial condition from a measurement of the solution on an arbitrary part of the boundary and at some arbitrary positive time. The key ingredient is the derivation of a Carleman-type estimate. The diffusion coefficient is assumed to be discontinuous across an interface with a monotonicity condition.
\end{abstract}

AMS 2000 subject classification: 35K05, 35R30.

\section{Introduction}

This article is devoted to the question of the identification of a diffusion coefficient, $c$, for a heat transfer problem in a bounded domain, with the main particularity that $c$ is discontinuous. Such regularity can be encountered in the case of embedded materials.

Let $\Omega \subset \mathbb{R}^{n}$ be a bounded connected open set. The set $\bar{\Omega}$ is assumed to be a $\mathscr{C}^{2}$ submanifold with boundary in $\mathbb{R}^{n}$ (see e.g. [12, Definition 1.2.1.2]). We set $\Gamma=\partial \Omega$. Let $\Omega_{0}$ and $\Omega_{1}$ be two non-empty open subsets of $\Omega$ such that

$$
\Omega_{0} \Subset \Omega \text {, and } \Omega_{1}=\Omega \backslash \bar{\Omega}_{0} .
$$

\footnotetext{
${ }^{1}$ Université Aix-Marseille I, ${ }^{2}$ Université Aix-Marseille II.

a assia@cmi .univ-mrs.fr, ${ }^{b}$ gaitan@cmi . univ-mrs.fr, ${ }^{c} j$ jerous@cmi.univ-mrs.fr
} 
We denote by $S=\bar{\Omega}_{0} \cap \bar{\Omega}_{1}$ the interface, which is assumed to be $\mathscr{C}^{2}$. We shall use the following notation $\Omega^{\prime}=\Omega_{0} \cup \Omega_{1}$. It should be emphasized here that the position of the interface itself is not assumed to be known.

Let $T>0$. We consider the following transmission problem for the heat equation:

$$
\begin{cases}\partial_{t} y-\nabla \cdot(c \nabla y)=0 & \text { in }(0, T) \times \Omega^{\prime}, \\ y(t, x)=h(t, x) & \text { on }(0, T) \times \Gamma, \\ \text { transmission conditions }(\mathrm{TC} 1) & \text { on }(0, T) \times S, \\ y(0, x)=y_{0}(x), & \text { in } \Omega,\end{cases}
$$

with

$$
y_{\mid[0, T] \times S_{0}}=y_{\mid[0, T] \times S_{1}}, \quad c_{0} \partial_{n} y_{\mid[0, T] \times S_{0}}=c_{1} \partial_{n} y_{\mid[0, T] \times S_{1}}
$$

where $f_{\left.\right|_{S_{i}}}$ is the trace on $S$ of $f_{\left.\right|_{\Omega_{i}}}$ and where

$$
c=\left\{\begin{array}{ll}
c_{0} & \text { in } \Omega_{0}, \\
c_{1} & \text { in } \Omega_{1},
\end{array} \quad \widetilde{c}= \begin{cases}\widetilde{c}_{0} & \text { in } \Omega_{0}, \\
\widetilde{c}_{1} & \text { in } \Omega_{1} .\end{cases}\right.
$$

The boundary condition $h(t, x)$ shall be kept fixed. The diffusion coefficient $c$ shall be kept independent of time, $t$. If we change the diffusion coefficient $c$ into $\widetilde{c}$ we let $\widetilde{y}$ be the solution of $(0.1)$ associated to $\widetilde{c}$ and $\widetilde{y}_{0}$ for initial condition. In the case studied here, the interface remains unchanged when changing coefficients. Its position is however not known.

We assume that we can measure both the normal flux $\partial_{n} \partial_{t} y$ on $\gamma \subset \partial \Omega$ on the time interval $\left(t_{0}, T\right)$ for some $t_{0} \in(0, T)$ and $\Delta y$ in $\Omega$ at time $T^{\prime} \in\left(t_{0}, T\right)$. In the case of piecewise constant diffusion coefficients, i.e. $c_{\mid \Omega_{i}}, i=0,1$, is constant, our main results are $(i)$ the injectivity of the map

$$
\begin{aligned}
L^{\infty}(\Omega) \times L^{2}(\Omega) & \rightarrow L^{2}\left(\left(t_{0}, T\right) \times \gamma\right) \times L^{2}(\Omega), \\
\left(c, y_{0}\right) & \mapsto\left(\partial_{n} \partial_{t} y, \Delta y\left(T^{\prime}\right)\right),
\end{aligned}
$$

(uniqueness); (ii) the stability for the diffusion coefficient, $c$ (Theorem 2.9): there exists $C>0$ such that

$$
|c-\widetilde{c}|_{L^{\infty}(\Omega)}^{2} \leq C\left|\partial_{n}\left(\partial_{t} y-\partial_{t} \widetilde{y}\right)\right|_{L^{2}((0, T) \times \gamma)}^{2}+C\left|\Delta y\left(T^{\prime}, .\right)-\Delta \widetilde{y}\left(T^{\prime}, .\right)\right|_{L^{2}\left(\Omega^{\prime}\right)}^{2}
$$

and (iii) the stability for the initial condition, $y_{0}$ (Theorem 4.5): there exists $C>0$ such that

$$
\left|y_{0}-\widetilde{y}_{0}\right|_{L^{2}(\Omega)} \leq C /\left|\ln \left(\left|(y-\widetilde{y})\left(T^{\prime}\right)\right|_{H^{2}\left(\Omega^{\prime}\right)}+\left|\partial_{n}\left(\partial_{t} y-\partial_{t} \widetilde{y}\right)\right|_{L^{2}((0, T) \times \gamma)}^{2}\right)\right|,
$$

for $\left|(y-\widetilde{y})\left(T^{\prime}\right)\right|_{H^{2}\left(\Omega^{\prime}\right)}+\left|\partial_{n}\left(\partial_{t} y-\partial_{t} \widetilde{y}\right)\right|_{L^{2}((0, T) \times \gamma)}^{2}$ sufficiently small. At the end of Section 2, we shall observe that we may only require the difference of the coefficients, $c-\widetilde{c}$, to be piecewise constant.

The key ingredient to these stability results is a global Carleman estimate for the operator $\partial_{t}-\nabla \cdot(c \nabla()$.$) and the open set \Omega$. 
The use of Carleman estimates to achieve uniqueness and stability results in inverse problems is now well-established. Some authors make use of local Carleman inequalities and deduce uniqueness and Hölder estimates (see [18],[17] and references cited therein). Others make use of global Carleman inequalities and deduce Lipschitz stability results (and hence uniqueness results). We shall follow this second approach. To our knowledge, this method was first used in [19] and then by others, e.g. [2]. For literature on Carleman estimates we refer to [13, Chapter VIII], [14, Section: 28.2-3] for local estimates and [16] for the parabolic case. For global estimates we refer to [10] and [9].

Stability results for parabolic equations are recent, to our knowledge, (see $[18],[8])$. Apart from [19] there are few results on Lipschitz stability, even for linear cases.

One of the main difficulties in the present problem is to deal with discontinuous diffusion coefficients. Controllability for such parabolic equations has been studied by [7]. Null-controllability property is proved via an observability inequality for the adjoint system, which is deduced in [7] from a global Carleman estimate yet assuming a monotonicity on the coefficients $c$ in connection to the observation location: roughly speaking, the observation zone has to be located in the region where the diffusion coefficient is the smallest. Here, to achieve a stability result we have to derive a Carleman estimate for the difference of the two solutions, $y, \widetilde{y}$. This difference is solution of a non-homogeneous parabolic equation (with discontinuous coefficient); because of the discontinuity of the diffusion coefficients it does not satisfy the appropriate transmission conditions (TC1), on the interfaces $S$, defined above. For this reason, under the same monotonicity assumption as in [7], we derive a peculiar Carleman estimate which includes additional interface terms (see Theorem 1.2).

To obtain a stability result, one has to 'manage' the dependence of some constants with respect to (w.r.t.) the parameters, $s$ and $\lambda$, that appear in the weight functions used in the Carleman estimate (see (1.4) in Section 1). The interface terms require some careful treatment. In particular, a stationary-phase argument is used to obtain a sufficiently sharp asymptotic estimates of these terms for $s$ and $\lambda$ large. Usually, stability estimate are obtained by letting the parameter $s$ become large. Here we also make use of the second parameter $\lambda$ (see Section 3).

As we are concerned with parabolic equations, we have to assume the observation of the solution occurs at some positive time, $T^{\prime}>0$. The suppression of this assumption remains an open problem and it appears in all articles deriving Lipschitz stability estimates from global Carleman inequalities (see the discussion in the introduction of [19]). At the end of section 2 we show that if the position of the interface $S$ is known, we can however localize in space the observation at time $T^{\prime}$.

The article is organized as follows. In Section 1 we derive a Carleman estimate adapted to our problem. In Section 2 we prove a stability result for the piecewise constant diffusion coefficient $c$ when one of the solutions, say $\widetilde{y}$, is in a particular class of solutions. In Section 3 we prove that this class is non empty. As mentioned above we also slightly relax the piecewise-constant condition by solely imposing that the difference of the coefficients be piecewise constant. In 
Section 4 we prove a stability result for the initial condition under some additional assumptions, in particular on the initial condition itself. Section 5 is devoted to a generalization of the results to a more complicated geometry, for instance allowing for more than two embedded materials. Appendix A provides some basic regularity properties for the solutions to parabolic equation with non-smooth coefficients and provides a technical lemma.

We now give some notations and important assumptions. We denote by $n$ the outward unit normal to $\Omega_{1}$ on $S$ and also the outward unit normal to $\Omega$ on $\Gamma$. Let $S_{0}$ (resp. $S_{1}$ ) be the side of the interface $S$ corresponding to the positive (resp. negative) direction of the normal $n$.

Note that we do not assume that $\Omega_{0}$ nor $\Omega_{1}$ is a connected open set. We shall however assume that they are formed with a finite number of connected open sets, say $\Omega_{0,1}, \ldots, \Omega_{0, p_{0}}$, and $\Omega_{1,1}, \ldots, \Omega_{1, p_{1}}, p_{0}, p_{1} \in \mathbb{N}$. We shall then denote by $S_{i j}$ the interface (possibly empty) between $\Omega_{0, i}$ and $\Omega_{1, j}$.

We make the following assumption

Assumption 0.1. The diffusion coefficient satisfies $c_{i}=c_{\left.\right|_{\Omega_{i}}} \in \mathscr{C}^{1}\left(\bar{\Omega}_{i}\right), i=$ 0,1 , and is independent of time $t$.

Assumption 0.2. $c_{\left.0\right|_{S}} \geq c_{\left.1\right|_{S}}$ and $0<c_{\min } \leq c(x) \leq c_{\max }, x \in \Omega^{\prime}$.

Remark 0.3. Assumption 0.1 will be significantly strengthened in Section 2 to obtain a stability result. Yet, for some of the results such as the Carleman estimate proved in Section 1 and the regularity properties proved in Section 3, which can be of some use elsewhere, Assumption 0.1 is sufficient.

We let $\gamma$ be a subset of the boundary $\Gamma$ satisfying

Assumption 0.4. The interior of $\gamma$ is non-empty with respect to the topology on $\Gamma$ induced by the Euclidean topology on $\mathbb{R}^{n}$. Each component of $\Omega_{1}$ contains part of the interior of $\gamma$ in its boundary.

Examples of situations in which Assumption 0.4 is satisfied are given in Figure 1.

To obtain a Carleman estimate we introduce a geometric assumption, following [7].

Assumption 0.5. Geometric Condition (GC)

We assume that there exist two disjoint open subsets $\mathcal{O}^{(1)}, \mathcal{O}^{(2)} \Subset \Omega_{0}$ and two vector fields, $\zeta^{(i)} \in \mathscr{C}^{1}\left(\bar{\Omega}_{0}, \mathbb{R}^{2}\right), i=1,2$, such that

$$
\begin{gathered}
\zeta^{(i)}(x) \cdot n(x)>0, \quad \forall x \in S, \quad i=1,2, \\
\zeta^{(i)}(x) \cdot n(x)>0, \quad \forall x \in \partial \mathcal{O}^{(i)}, \quad i=1,2, \\
\zeta^{(i)}(x) \neq 0, \quad \forall x \in \Omega_{0} \backslash O^{(i)}, \quad i=1,2
\end{gathered}
$$

( $n$ is the outward unit normal to $\Omega_{1}$ on $S$ and the inward unit normal to $O^{(i)}$ on $\left.\partial O^{(i)}, i=1,2\right)$. Let $x^{(i)}$ be the integral curves of $\zeta^{(i)}$, i.e.

$$
\left\{\begin{array}{l}
\frac{d x^{(i)}(t)}{d t}=\zeta^{(i)}\left(x^{(i)}(t)\right), \quad t>0, \\
x^{(i)}(0)=x_{0}, \quad x_{0} \in S .
\end{array}\right.
$$



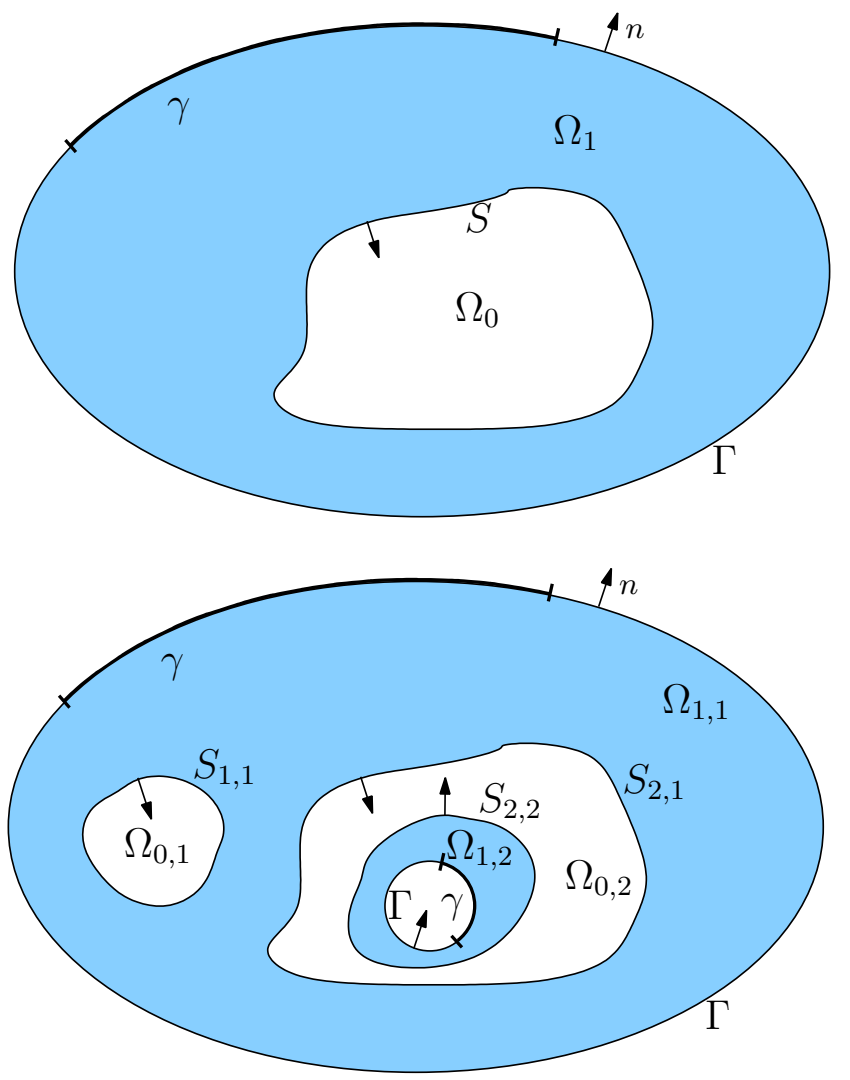

Figure 1: Geometric situations in which Assumption 0.4 and the geometric condition (GC) are satisfied. Shaded is $\Omega_{1}$. Arrows represent the normal unit vector $n$. 
We also assume that there exists $\mathcal{T}>0$ such that for all $x_{0} \in S$, there exists $t^{(i)}\left(x_{0}\right)<\mathcal{T}$ satisfying

$$
\begin{gathered}
x^{(i)}(t) \in \Omega_{0} \backslash O^{(i)}, \quad \text { for } 0<t<t^{(i)}\left(x_{0}\right), \quad x_{0} \in S, \quad i=1,2, \\
x^{(i)}\left(t^{(i)}\left(x_{0}\right)\right) \in \partial \mathcal{O}^{(i)}, \quad \text { for } x_{0} \in S, \quad i=1,2 .
\end{gathered}
$$

Note that in Assumption 0.5, there is no restriction to having $\Omega_{0}$ composed with $p_{0}$ components. The examples given in Figure 1 satisfy Assumption 0.5.

We denote by $W^{m, p}(\Omega), m \in \mathbb{N}, 1 \leq p \leq \infty$, the usual Sobolev space defined by

$$
W^{m, p}=\left\{u \in L^{p}(\Omega) ; \partial^{\alpha} u \in L^{p}(\Omega) \text { for }|\alpha| \leq m\right\},
$$

where $\alpha=\left(\alpha_{1}, \ldots, \alpha_{n}\right)$ is a multi-index and differentiation is to be understood in the weak sense. As usual we write $H^{m}(\Omega)=W^{m, 2}(\Omega)$. For the definition of $W^{r, p}$, for $r \in \mathbb{R} \backslash \mathbb{N}$ we refer for instance to [1].

\section{A Carleman estimate}

We prove here a Carleman-type estimate with a boundary term on $\gamma$ in the r.h.s. of the estimate. For this purpose we shall first introduce a particular type of weight functions, which are constructed using the following lemma.

Lemma 1.1. Assume that there exist two disjoint open subsets $\mathcal{O}^{(1)}, \mathcal{O}^{(2)} \Subset \Omega_{0}$ satisfying $(G C)$. Let $\gamma$ be a subset of $\Gamma=\partial \Omega$ satisfying Assumption 0.4 and $B^{(i)}$ and $\widetilde{B}^{(i)}, i=1,2$ be open balls such that $B^{(1)} \Subset \widetilde{B}^{(1)} \Subset \mathcal{O}^{(1)}$ and $B^{(2)} \Subset \widetilde{B}^{(2)} \Subset$ $\mathcal{O}^{(2)}$. Then there exists two functions $\widetilde{\beta}^{(1)}$ and $\widetilde{\beta}^{(2)}$ such that

$$
\widetilde{\beta}^{(1)}(x)=\left\{\begin{array}{ll}
\widetilde{\beta}_{0}^{(1)} & \text { in } \Omega_{0}, \\
\widetilde{\beta}_{1} & \text { in } \bar{\Omega}_{1},
\end{array} \quad \widetilde{\beta}^{(2)}(x)= \begin{cases}\widetilde{\beta}_{0}^{(2)} & \text { in } \Omega_{0}, \\
\widetilde{\beta}_{1} & \text { in } \bar{\Omega}_{1},\end{cases}\right.
$$

and the functions $\widetilde{\beta}_{0}^{(1)}, \widetilde{\beta}_{0}^{(2)}$, and $\widetilde{\beta}_{1}$ satisfy the following properties: $\widetilde{\beta}_{1} \in$ $\mathscr{C}^{2}\left(\bar{\Omega}_{1}\right), \widetilde{\beta}_{1}>0$ in $\Omega_{1}$, and

$$
\begin{gathered}
\widetilde{\beta}_{1}=0 \text { on } \Gamma \backslash \gamma, \quad \partial_{n} \widetilde{\beta}_{1}<0 \text { on } \Gamma \backslash \gamma, \\
\widetilde{\beta}_{1}=2 \text { on } S, \quad \partial_{n} \widetilde{\beta}_{1}<0 \text { on } S,
\end{gathered}
$$

and

$$
\left|\nabla \widetilde{\beta}_{1}\right|>0 \text { in } \bar{\Omega}_{1}
$$

for $i=1,2, \widetilde{\beta}_{0}^{(i)} \in \mathscr{C}^{2}\left(\bar{\Omega}_{0}\right), \widetilde{\beta}_{0}^{(i)}>0$ in $\Omega_{0}$,

$$
\begin{gathered}
\widetilde{\beta}_{0}^{(i)}=\widetilde{\beta}_{1}=2 \text { on } S, \quad i=1,2, \\
c_{0} \partial_{n} \widetilde{\beta}_{0}^{(i)}=c_{1} \partial_{n} \widetilde{\beta}_{1} \text { on } S, \quad i=1,2,
\end{gathered}
$$

$$
\begin{aligned}
& \widetilde{\beta}_{0}^{(1)} \geq 2 \widetilde{\beta}_{0}^{(2)} \text { in } \widetilde{B}^{(2)}, \\
& \widetilde{\beta}_{0}^{(2)} \geq 2 \widetilde{\beta}_{0}^{(1)} \text { in } \widetilde{B}^{(1)},
\end{aligned}
$$

and

$$
\left|\nabla \widetilde{\beta}_{0}^{(i)}\right|>0 \text { in } \bar{\Omega}_{0} \backslash B^{(i)}, \quad i=1,2 .
$$




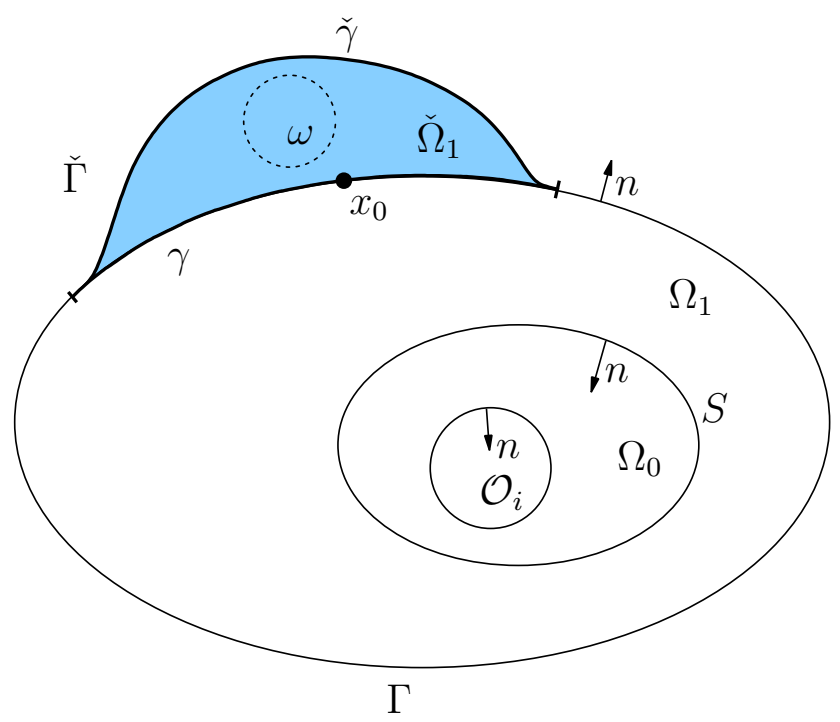

Figure 2: Geometrical situation for the proof Lemma 1.1. The shaded area represents $\check{\Omega}_{1} \backslash \Omega_{1}=\check{\Omega} \backslash \Omega$.

Proof. For the construction of $\widetilde{\beta}_{0}^{(i)}, i=1,2$, supported in the connected components of $\Omega_{0}$, we refer to [7, Lemma 3.2]. We briefly show how the function $\widetilde{\beta}_{1}$ is constructed. In actuality, the procedure described here has to be performed in each connected component of $\Omega_{1}$, which is possible since each component contains part of the interior of $\gamma$ in its boundary by Assumption 0.4.

Let $x_{0}$ be in the interior of $\gamma$. We can enlarge the open set $\Omega_{1}$ locally around $x_{0}$ while preserving the $\mathscr{C}^{2}$ regularity of the boundary. Such a procedure is performed in a neighborhood $U$ of $x_{0}$ such that $U \cap \Gamma \subset \gamma$. (This can be done by locally straightening out the boundary $\gamma$ as $\bar{\Omega}$ is assumed to be a $\mathscr{C}^{2}$ submanifold with boundary in $\mathbb{R}^{n}[12$, Definition 1.2.1.2]). This enlarging procedure only affects $\gamma$ and leaves $\Gamma \backslash \gamma$ untouched. We call the new boundary $\check{\Gamma}$. We denote $\check{\Omega}_{1}$ the extension of $\Omega_{1}$ and $\check{\Omega}$ that of $\Omega\left(\Omega_{1} \subset \check{\Omega}_{1}, \Omega \subset \check{\Omega}\right.$ and $\check{\Gamma}=\partial \check{\Omega})$. Let $\omega$ be an open subset such that $\omega \Subset \check{\Omega}_{1} \backslash \Omega_{1}$. The geometry we describe here is illustrated in Figure 2. Following [7, 10], there exists $\mu \in \mathscr{C}^{2}(\bar{\Omega})$ that satisfies

$$
\begin{gathered}
\mu=0, \quad \partial_{n} \mu<0 \text { on } \check{\Gamma}, \\
\mu=2, \quad c_{0} \partial_{n} \widetilde{\beta}_{0}^{(i)}=c_{1} \partial_{n} \mu<0 \text { on } S, i=1,2 . \\
|\nabla \mu|>0 \text { in } \check{\Omega}_{1} \backslash \omega
\end{gathered}
$$

The function $\widetilde{\beta}_{1}:=\mu_{\left.\right|_{\bar{\Omega}_{1}}}$ satisfies the required properties.

Choosing two functions $\widetilde{\beta}^{(i)}, i=1,2$, as in the previous lemma, we introduce $\beta^{(i)}=\widetilde{\beta}^{(i)}+K$ with $K=m\left\|\widetilde{\beta}^{(1)}\right\|_{\infty}=m\left\|\widetilde{\beta^{(2)}}\right\|_{\infty}$ and $m>1$. For $\lambda>0$ and 
$t \in\left(t_{0}, T\right)$, we define the following weight functions

$$
\varphi^{(i)}(t, x)=\frac{e^{\lambda \beta^{(i)}(x)}}{\left(t-t_{0}\right)(T-t)}, \quad \eta^{(i)}(t, x)=\frac{e^{\lambda \bar{\beta}}-e^{\lambda \beta^{(i)}(x)}}{\left(t-t_{0}\right)(T-t)}, i=1,2,
$$

with $\bar{\beta}=2 m\left\|\widetilde{\beta}^{(i)}\right\|_{\infty}, i=1,2$ (see [7],[9]). We let $t_{0} \in(0, T)$ and we set $Q=\left(t_{0}, T\right) \times \Omega, Q^{\prime}=\left(t_{0}, T\right) \times \Omega^{\prime}$ and recall that $\Omega^{\prime}=\Omega_{0} \cup \Omega_{1}$.

Let $g \in H^{1}\left(\left[t_{0}, T\right], H^{\frac{1}{2}}(S)\right)$. We introduce transmission conditions (TC2) on the interval $\left[t_{0}, T\right]$ which reads

$$
\begin{aligned}
& q_{\mid\left[t_{0}, T\right] \times S_{0}}=q_{\mid\left[t_{0}, T\right] \times S_{1}}, \\
& c_{0} \partial_{n} q_{\mid\left[t_{0}, T\right] \times S_{0}}=c_{1} \partial_{n} q_{\mid\left[t_{0}, T\right] \times S_{1}}+g(t, x),
\end{aligned}
$$

for a function $q$ which is $H^{2}$ in each open set $\Omega_{i}, i=0,1$.

We introduce

$$
\begin{array}{r}
\aleph_{g}=\left\{q \in H^{1}\left(t_{0}, T, H_{0}^{1}(\Omega)\right) ; q_{\left.\right|_{\left(t_{0}, T\right) \times \Omega_{i}}} \in L^{2}\left(t_{0}, T, H^{2}\left(\Omega_{i}\right)\right), i=0,1,\right. \\
\text { and } q \text { satisfies }(\mathrm{TC} 2) \text { a.e. w.r.t. } t\} .
\end{array}
$$

Theorem 1.2. Let $\gamma$ be a subset of the boundary $\Gamma$ of an open set $\Omega$ of $\mathbb{R}^{n}$ that satisfies Condition (GC), and $\gamma$ satisfies Assumption 0.4. Let c satisfy Assumptions 0.1 and 0.2. Assume further that $c_{\left.0\right|_{S}}-c_{\left.1\right|_{S}} \geq \Delta>0$. Let $g \in H^{1}\left(t_{0}, T, H^{\frac{1}{2}}(S)\right)$. There exists $\lambda_{1}=\lambda_{1}\left(\Omega, \gamma, \mathcal{O}^{(1)}, \mathcal{O}^{(2)}, c_{\min }, c_{\max }, \Delta\right)>0$, $s_{1}=s_{1}\left(\lambda_{1}\right)>0$ and a positive constant $C=C\left(\Omega, \gamma, \mathcal{O}^{(1)}, \mathcal{O}^{(2)}, c_{\min }, c_{\max }, \Delta\right)$ so that the following estimate holds

$$
\begin{aligned}
& \left|M_{1}^{(1)}\left(e^{-s \eta^{(1)}} q\right)\right|_{L^{2}\left(Q^{\prime}\right)}^{2}+\left|M_{1}^{(2)}\left(e^{-s \eta^{(2)}} q\right)\right|_{L^{2}\left(Q^{\prime}\right)}^{2} \\
& +\left|M_{2}^{(1)}\left(e^{-s \eta^{(1)}} q\right)\right|_{L^{2}\left(Q^{\prime}\right)}^{2}+\left|M_{2}^{(2)}\left(e^{-s \eta^{(2)}} q\right)\right|_{L^{2}\left(Q^{\prime}\right)}^{2} \\
& +s \lambda^{2} \iint_{Q}\left(e^{-2 s \eta^{(1)}} \varphi^{(1)}+e^{-2 s \eta^{(2)}} \varphi^{(2)}\right)|\nabla q|^{2} d x d t \\
& +s^{3} \lambda^{4} \iint_{Q}\left(e^{-2 s \eta^{(1)}} \varphi^{(1)^{3}}+e^{-2 s \eta^{(2)}} \varphi^{(2)^{3}}\right)|q|^{2} d x d t \\
& \leq C\left[s \lambda \int_{t_{0}}^{T} \int_{\gamma}\left(e^{-2 s \eta^{(1)}} \varphi^{(1)}+e^{-2 s \eta^{(2)}} \varphi^{(2)}\right)\left|\partial_{n} q\right|^{2} d \sigma d t\right. \\
& +\iint_{Q^{\prime}}\left(e^{-2 s \eta^{(1)}}+e^{-2 s \eta^{(2)}}\right)\left|\partial_{t} q \pm \nabla \cdot(c \nabla q)\right|^{2} d x d t \\
& +s \lambda \int_{t_{0}}^{T} \int_{S}\left(e^{-2 s \eta^{(1)}} \varphi^{(1)}+e^{-2 s \eta^{(2)}} \varphi^{(2)}\right)|g|^{2} d \sigma d t \\
& +\int_{t_{0}}^{T} \int_{S}\left(e^{-2 s \eta^{(1)}} \varphi^{(1)^{4}}+e^{-2 s \eta^{(2)}} \varphi^{(2)^{4}}\right)|g|^{2} d \sigma d t \\
& \left.+s^{-2} \int_{t_{0}}^{T} \int_{S}\left(e^{-2 s \eta^{(1)}}+e^{-2 s \eta^{(2)}}\right)\left|\partial_{t} g\right|^{2} d \sigma d t\right],
\end{aligned}
$$

for $s \geq s_{1}, \lambda \geq \lambda_{1}$ and for all $q \in \aleph_{g}$, with $M_{1}$ and $M_{2}$ to be defined in (1.9)-(1.10). 
We recall that $\Omega^{\prime}=\Omega_{0} \cup \Omega_{1}$ and $Q^{\prime}=\Omega^{\prime} \times(0, T)$ For a function $\rho$ with a trace on the interface $S$, from both sides, defined in some sense, we shall denote $\rho_{i}$ the trace of $\rho_{\Omega_{\Omega_{i}}}$ on $S, i=0,1$, when there is no ambiguity; in the case $\rho_{0}=\rho_{1}$ we shall simply write $\rho$. We shall use the notation $[\rho]_{S}=\rho_{0}-\rho_{1}$ for the jump of $\rho$ across the interface $S$. We shall adopt Einstein's summation convention for repeated indices.

Proof. We consider $s>0$ and $q \in \aleph_{g}$. Let us set $f=\partial_{t} q+\nabla \cdot(c \nabla q)$ (we treat the case of the operator $\partial_{t}+\nabla \cdot(c \nabla)$ in the proof; the other case can be treated similarly). Then $f \in L^{2}\left(Q^{\prime}\right)$ (because of transmission conditions (TC2), observe that $q$ is not in the domain of the operator $\nabla \cdot(c \nabla)$ ).

In the first part of the proof we shall write $\eta, \varphi, M_{1}$, etc, in place of $\eta^{(i)}, \varphi^{(i)}$, $M_{1}^{(i)}$, etc, $i=1,2$, and treat the two cases at a time. We set $\psi=e^{-s \eta} q$. We observe that $\psi\left(t_{0}\right)=\psi(T)=0$ and since $q$ satisfies transmission conditions (TC2) (and $q(t, .)_{\left.\right|_{\Omega_{i}}} \in H^{2}\left(\Omega_{i}\right)$ a.e. w.r.t. $t$ ), we have (a.e. w.r.t. $t$ )

$$
\begin{aligned}
c_{0} \partial_{n} \psi_{\left.0\right|_{S}}(t, .) & =c_{1} \partial_{n} \psi_{\left.1\right|_{S}}(t, .)+g_{s}(t, .) \text { on } S, \\
\nabla_{\tau} \psi_{\left.0\right|_{S}}(t, .) & =\nabla_{\tau} \psi_{\left.1\right|_{S}}(t, .) \quad \text { on } S, \\
\psi_{\left.0\right|_{S}}(t, .) & =\psi_{\left.\right|_{S}}(t, .) \quad \text { on } S,
\end{aligned}
$$

where $g_{s}=e^{-s \eta} g$ and $\nabla_{\tau}$ denotes the component of the gradient that is tangential to $S$.

The function $\psi$ satisfies in each $\Omega_{i}, i=0,1$,

$$
M_{1} \psi+M_{2} \psi=f_{s}
$$

with

$$
\begin{aligned}
M_{1} \psi & =\nabla \cdot(c \nabla \psi)+s^{2} \lambda^{2} \varphi^{2}|\nabla \beta|^{2} c \psi+s\left(\partial_{t} \eta\right) \psi, \\
M_{2} \psi & =\partial_{t} \psi-2 s \lambda \varphi c \nabla \beta \cdot \nabla \psi-2 s \lambda^{2} \varphi c|\nabla \beta|^{2} \psi, \\
f_{s} & =e^{-s \eta} f+s \lambda \varphi \nabla \cdot(c \nabla \beta) \psi-s \lambda^{2} \varphi c|\nabla \beta|^{2} \psi .
\end{aligned}
$$

We have

$$
\left|M_{1} \psi\right|_{L^{2}\left(Q^{\prime}\right)}^{2}+\left|M_{2} \psi\right|_{L^{2}\left(Q^{\prime}\right)}^{2}+2\left(M_{1} \psi, M_{2} \psi\right)_{L^{2}\left(Q^{\prime}\right)}=\left|f_{s}\right|_{L^{2}\left(Q^{\prime}\right)}^{2}
$$

With the same notations as in [7, Theorem 3.3], we write $\left(M_{1} \psi, M_{2} \psi\right)_{L^{2}\left(Q^{\prime}\right)}$ as a sum of 9 terms $I_{i j}, 1 \leq i, j \leq 3$, where $I_{i j}$ is the inner product of the $i$ th term in the expression of $M_{1} \psi$ and the $j$ th term in the expression of $M_{2} \psi$.

As compared to the proof of the Carleman estimate in [7, Theorem 3.3] we only need to adjust the computation of $I_{11}, I_{12}$ and $I_{13}$ to the present case. In fact the other terms do not involve transmission conditions (1.6) in their computation and thus remain unchanged from the terms obtained in [7].

The term $I_{11}$ follows as

$$
\begin{aligned}
& I_{11}=\iint_{Q^{\prime}} \nabla \cdot(c \nabla \psi) \partial_{t} \psi d x d t \\
& \quad=-\iint_{Q^{\prime}} c \nabla \psi \cdot \partial_{t}(\nabla \psi) d x d t+\sum_{i=0,1}(-1)^{i+1} \int_{t_{0}}^{T} \int_{S} c_{i} \partial_{n} \psi_{i} \partial_{t} \psi d \sigma d t
\end{aligned}
$$


by integration by parts; the surface integral on $\Gamma$ vanishes since $\partial_{t} \psi=0$ there. Noting that $\nabla \psi \cdot \partial_{t}(\nabla \psi)=\frac{1}{2} \partial_{t}\left(|\nabla \psi|^{2}\right)$, the first term vanishes since $\psi$, and thus $\nabla \psi$, vanish at $t=t_{0}$ and $t=T$ and $c$ is independent of $t$. For the remaining surface terms we use (1.6), which yields

$$
I_{11}=-\int_{t_{0}}^{T} \int_{S} g_{s} \partial_{t} \psi d \sigma d t=\int_{t_{0}}^{T} \int_{S} \partial_{t}\left(g_{s}\right) \psi d \sigma d t
$$

since $g_{s} \in H^{1}\left(t_{0}, T, H^{\frac{1}{2}}(S)\right)$.

The term $I_{12}$ is given by

$$
\begin{aligned}
& I_{12}=-2 s \lambda \iint_{Q^{\prime}} \varphi \nabla \cdot(c \nabla \psi) c \nabla \beta \cdot \nabla \psi d x d t \\
& =2 s \lambda \iint_{Q^{\prime}} c \nabla \psi \cdot \nabla(\varphi c \nabla \beta \cdot \nabla \psi) d x d t-2 s \lambda \int_{t_{0}}^{T} \int_{\Gamma} \varphi c^{2}(\nabla \beta \cdot \nabla \psi)\left(\partial_{n} \psi\right) d \sigma d t \\
& \quad+2 s \lambda \sum_{i=0,1}(-1)^{i} \int_{t_{0}}^{T} \int_{S} \varphi c_{i}^{2}\left(\nabla \beta_{i} \cdot \nabla \psi_{i}\right)\left(\partial_{n} \psi_{i}\right) d \sigma d t .
\end{aligned}
$$

This integration by parts is justified since $\psi(t,$.$) is in H^{2}$ in each $\Omega_{i}, i=0,1$. Denoting by $I_{12}^{\prime}$ the remaining volume integral, we obtain

$$
\begin{array}{r}
I_{12}^{\prime}=2 s \lambda^{2} \iint_{Q^{\prime}} \varphi c^{2}|\nabla \psi \cdot \nabla \beta|^{2} d x d t+2 s \lambda \iint_{Q^{\prime}} \varphi c \partial_{x_{i}}\left(c \partial_{x_{j}} \beta\right) \partial_{x_{i}} \psi \partial_{x_{j}} \psi d x d t \\
+s \lambda \iint_{Q^{\prime}} \varphi c^{2} \partial_{x_{j}} \beta \partial_{x_{j}}|\nabla \psi|^{2} d x d t .
\end{array}
$$

We further compute the last volume integral, denoted by $I_{12}^{\prime \prime}$. Observe that $|\nabla \psi|_{\left.\right|_{\Omega_{i}}}^{2}$ is in $W^{1,1}\left(\Omega_{i}\right)$ since $\psi_{\left.\right|_{\Omega_{i}}}(t,.) \in H^{2}\left(\Omega_{i}\right), i=0,1$. This allows to further integrate by parts, since $\left(c^{2} \varphi \partial_{x_{j}} \beta\right)_{\left.\right|_{\Omega_{i}}} \in \mathscr{C}^{1}\left(\Omega_{i}\right), i=0,1$, and yields

$$
\begin{array}{r}
I_{12}^{\prime \prime}=-s \lambda \iint_{Q^{\prime}} \partial_{x_{j}}\left(\varphi c^{2} \partial_{x_{j}} \beta\right)|\nabla \psi|^{2} d x d t+s \lambda \int_{t_{0}}^{T} \int_{\Gamma} \varphi c^{2} \partial_{n} \beta|\nabla \psi|^{2} d \sigma d t \\
+s \lambda \sum_{i=0,1}(-1)^{i+1} \int_{t_{0}}^{T} \int_{S} \varphi c_{i}^{2} \partial_{n} \beta_{i}\left|\nabla \psi_{i}\right|^{2} d \sigma d t .
\end{array}
$$

The remaining volume integral can be further expanded into

$$
I_{12}^{\prime \prime \prime}=-s \lambda \iint_{Q^{\prime}} \varphi \partial_{x_{j}}\left(c^{2} \partial_{x_{j}} \beta\right)|\nabla \psi|^{2} d x d t-s \lambda^{2} \iint_{Q^{\prime}} \varphi c^{2}|\nabla \beta|^{2}|\nabla \psi|^{2} d x d t .
$$

Collecting the surface integrals in a term denoted by $J_{12}$ we find

$$
\begin{array}{r}
I_{12}=-s \lambda^{2} \iint_{Q^{\prime}} \varphi c^{2}|\nabla \beta|^{2}|\nabla \psi|^{2} d x d t+2 s \lambda^{2} \iint_{Q^{\prime}} c^{2} \varphi|\nabla \psi \cdot \nabla \beta|^{2} d x d t \\
+X_{1}+J_{12},
\end{array}
$$


where

$$
\begin{aligned}
X_{1}=2 s \lambda \iint_{Q^{\prime}} \varphi c \partial_{x_{i}}\left(c \partial_{x_{j}} \beta\right) \partial_{x_{i}} \psi \partial_{x_{j}} \psi & d x d t \\
& -s \lambda \iint_{Q^{\prime}} \varphi \partial_{x_{j}}\left(c^{2} \partial_{x_{j}} \beta\right)|\nabla \psi|^{2} d x d t .
\end{aligned}
$$

We now observe that since $\beta$ is constant on $S$ we have

$$
\left(\nabla \beta \cdot \nabla \psi_{i}\right)_{\left.\right|_{S}}=\left(\partial_{n} \beta \partial_{n} \psi_{i}\right)_{\left.\right|_{S}}, \quad i=0,1 .
$$

Writing $|\nabla \psi|^{2}=\left|\nabla_{\tau} \psi\right|^{2}+\left|\partial_{n} \psi\right|^{2}$ we find

$$
\begin{aligned}
& J_{12}=s \lambda \sum_{i=0,1}(-1)^{i} \int_{t_{0}}^{T} \int_{S} \varphi c_{i}^{2} \partial_{n} \beta_{i}\left|\partial_{n} \psi_{i}\right|^{2} d \sigma d t \\
& -s \lambda \sum_{i=0,1}(-1)^{i} \int_{t_{0}}^{T} \int_{S} \varphi c_{i}^{2} \partial_{n} \beta_{i}\left|\nabla_{\tau} \psi_{i}\right|^{2} d \sigma d t-s \lambda \int_{t_{0}}^{T} \int_{\Gamma} \varphi c^{2} \partial_{n} \beta\left|\partial_{n} \psi\right|^{2} d \sigma d t
\end{aligned}
$$

where we have used that $\psi_{\left.\right|_{\Sigma}}$ is constant. Recall that $\nabla_{\tau} \psi_{0}=\nabla_{\tau} \psi_{1}$, and that $c_{0} \partial_{n} \beta_{0}=c_{1} \partial_{n} \beta_{1}$ on $S$. From transmission conditions (TC2) we have

$$
\left|c_{0} \partial_{n} \psi_{0}\right|^{2}=\left|c_{1} \partial_{n} \psi_{1}\right|^{2}+\left|g_{s}\right|^{2}+2\left(c_{1}\left(\partial_{n} \psi_{1}\right) g_{s}\right), \quad \text { on } S \text {. }
$$

We thus obtain

$$
\begin{aligned}
& J_{12}=s \lambda \int_{t_{0}}^{T} \int_{S} \varphi\left[\partial_{n} \beta\right]_{S}\left|c_{1} \partial_{n} \psi_{1}\right|^{2} d \sigma d t \\
& +s \lambda \int_{t_{0}}^{T} \int_{S} \varphi \partial_{n} \beta_{0}\left|g_{s}\right|^{2} d \sigma d t-s \lambda \int_{t_{0}}^{T} \int_{S} \varphi[c]_{S}\left(c \partial_{n} \beta\right)\left|\nabla_{\tau} \psi\right|^{2} d \sigma d t \\
& -s \lambda \int_{t_{0}}^{T} \int_{\Gamma} \varphi c^{2} \partial_{n} \beta\left|\partial_{n} \psi\right|^{2} d \sigma d t+Y_{1},
\end{aligned}
$$

with

$$
Y_{1}=2 s \lambda \int_{t_{0}}^{T} \int_{S} \varphi c_{1} \partial_{n} \psi_{1} \partial_{n} \beta_{0} g_{s} d \sigma d t
$$

We thus have

$$
\begin{aligned}
I_{12}= & -s \lambda^{2} \iint_{Q^{\prime}} \varphi c^{2}|\nabla \beta|^{2}|\nabla \psi|^{2} d x d t+2 s \lambda^{2} \iint_{Q^{\prime}} c^{2} \varphi|\nabla \psi \cdot \nabla \beta|^{2} d x d t \\
& +s \lambda \int_{t_{0}}^{T} \int_{S} \varphi\left[\partial_{n} \beta\right]_{S}\left|c_{1} \partial_{n} \psi_{1}\right|^{2} d \sigma d t+s \lambda \int_{t_{0}}^{T} \int_{S} \varphi \partial_{n} \beta_{0}\left|g_{s}\right|^{2} d \sigma d t \\
& -s \lambda \int_{t_{0}}^{T} \int_{S} \varphi[c]_{S}\left(c \partial_{n} \beta\right)\left|\nabla_{\tau} \psi\right|^{2} d \sigma d t-s \lambda \int_{t_{0}}^{T} \int_{\Gamma} \varphi c^{2} \partial_{n} \beta\left|\partial_{n} \psi\right|^{2} d \sigma d t \\
& +X_{1}+Y_{1} .
\end{aligned}
$$


The term $I_{13}$ is given by

$$
\begin{aligned}
I_{13}=-2 s \lambda^{2} \iint_{Q^{\prime}} \varphi \nabla \cdot(c \nabla \psi) c|\nabla \beta|^{2} \psi d x d t \\
=2 s \lambda^{2} \iint_{Q^{\prime}} c \nabla \psi \cdot \nabla\left(\varphi c|\nabla \beta|^{2} \psi\right) d x d t \\
\quad+2 s \lambda^{2} \sum_{i=0,1}(-1)^{i} \int_{t_{0}}^{T} \int_{S} \varphi\left(c_{i} \partial_{n} \psi_{i}\right) c_{i}\left|\nabla \beta_{i}\right|^{2} \psi d \sigma d t
\end{aligned}
$$

where we have used that $\psi_{\left.\right|_{\Gamma}}=0$. Expanding the integrand in the volume integral and using (TC2) in the surface term we obtain

$$
I_{13}=2 s \lambda^{2} \iint_{Q^{\prime}} \varphi c^{2}|\nabla \beta|^{2}|\nabla \psi|^{2} d x d t+X_{2}+Y_{2},
$$

where

$$
\begin{aligned}
X_{2}=2 s \lambda^{2} \iint_{Q^{\prime}} \varphi & c \nabla \psi \cdot \nabla\left(c|\nabla \beta|^{2}\right) \psi d x d t \\
& +2 s \lambda^{3} \iint_{Q^{\prime}} \varphi c^{2} \nabla \psi \cdot \nabla \beta|\nabla \beta|^{2} \psi d x d t \\
& \quad+2 s \lambda^{2} \int_{t_{0}}^{T} \int_{S} \varphi\left(c \partial_{n} \beta\right)\left[\partial_{n} \beta\right]_{S}\left(c_{1} \partial_{n} \psi_{1}\right) \psi d \sigma d t
\end{aligned}
$$

since $\nabla_{\tau} \beta_{\left.\right|_{S}}=0$ and

$$
Y_{2}=2 s \lambda^{2} \int_{t_{0}}^{T} \int_{S} \varphi c_{0}\left(\partial_{n} \beta_{0}\right)^{2} g_{s} \psi d \sigma d t
$$

Following the proof of Theorem 3.3 in [7] we find

$$
I_{21}=\frac{1}{2} s^{2} \lambda^{2} \iint_{Q^{\prime}} \varphi^{2} c|\nabla \beta|^{2} \partial_{t}|\psi|^{2} d x d t=-\frac{1}{2} s^{2} \lambda^{2} \iint_{Q^{\prime}} \partial_{t}\left(\varphi^{2}\right) c|\nabla \beta|^{2}|\psi|^{2} d x d t,
$$

and

$$
\begin{aligned}
& I_{22}=-s^{3} \lambda^{3} \iint_{Q^{\prime}} \varphi^{3} c^{2}|\nabla \beta|^{2} \nabla \beta \cdot \nabla\left(|\psi|^{2}\right) d x d t \\
= & 3 s^{3} \lambda^{4} \iint_{Q^{\prime}} \varphi^{3} c^{2}|\nabla \beta|^{4}|\psi|^{2} d x d t+s^{3} \lambda^{3} \int_{t_{0}}^{T} \int_{S} \varphi^{3}\left|c \partial_{n} \beta\right|^{2}\left[\partial_{n} \beta\right]_{S}|\psi|^{2} d \sigma d t+X_{3},
\end{aligned}
$$

with $X_{3}$ given by

$$
X_{3}=s^{3} \lambda^{3} \iint_{Q^{\prime}} \varphi^{3} \nabla \cdot\left(c^{2}|\nabla \beta|^{2} \nabla \beta\right)|\psi|^{2} d x d t .
$$

The terms $I_{23}, I_{31}$ are given by

$$
I_{23}=-2 s^{3} \lambda^{4} \iint_{Q^{\prime}} \varphi^{3} c^{2}|\nabla \beta|^{4}|\psi|^{2} d x d t,
$$




$$
I_{31}=\frac{1}{2} s \iint_{Q^{\prime}} \partial_{t} \eta \partial_{t}\left(|\psi|^{2}\right) d x d t=-\frac{1}{2} s \iint_{Q^{\prime}} \partial_{t}^{2} \eta|\psi|^{2} d x d t .
$$

The term $I_{32}$ is given by

$$
\begin{aligned}
I_{32}=-s^{2} \lambda \iint_{Q^{\prime}} \varphi\left(\partial_{t} \eta\right) c \nabla \beta \cdot \nabla\left(|\psi|^{2}\right) & d x d t \\
& =s^{2} \lambda^{2} \iint_{Q^{\prime}} \varphi\left(\partial_{t} \eta\right) c|\nabla \beta|^{2}|\psi|^{2} d x d t \\
& +s^{2} \lambda \iint_{Q^{\prime}} \varphi \nabla \cdot\left(\left(\partial_{t} \eta\right) c \nabla \beta\right)|\psi|^{2} d x d t,
\end{aligned}
$$

since $\psi_{\mid S_{0}}=\psi_{\mid S_{1}}$. Finally the term $I_{33}$ is given by

$$
I_{33}=-2 s^{2} \lambda^{2} \iint_{Q^{\prime}} \varphi c\left(\partial_{t} \eta\right)|\nabla \beta|^{2}|\psi|^{2} d x d t .
$$

Collecting the terms $I_{i j}$ just computed we obtain

$$
\begin{aligned}
& \text { (1.13) }\left|M_{1} \psi\right|_{L^{2}\left(Q^{\prime}\right)}^{2}+\left|M_{2} \psi\right|_{L^{2}\left(Q^{\prime}\right)}^{2}+4 s \lambda^{2} \iint_{Q^{\prime}} c^{2} \varphi|\nabla \psi \cdot \nabla \beta|^{2} d x d t \\
& +2 s \lambda^{2} \iint_{Q^{\prime}} \varphi c^{2}|\nabla \beta|^{2}|\nabla \psi|^{2} d x d t+2 s^{3} \lambda^{4} \iint_{Q^{\prime}} \varphi^{3} c^{2}|\nabla \beta|^{4}|\psi|^{2} d x d t \\
& +2 s \lambda \int_{t_{0}}^{T} \int_{S} \varphi\left[\partial_{n} \beta\right]_{S}\left|c_{1} \partial_{n} \psi_{1}\right|^{2} d \sigma d t-2 s \lambda \int_{t_{0}}^{T} \int_{\Gamma} \varphi c^{2} \partial_{n} \beta\left|\partial_{n} \psi\right|^{2} d \sigma d t \\
& -2 s \lambda \int_{t_{0}}^{T} \int_{S} \varphi[c]_{S}\left(c \partial_{n} \beta\right)\left|\nabla_{\tau} \psi\right|^{2} d \sigma d t+2 s^{3} \lambda^{3} \int_{t_{0}}^{T} \int_{S} \varphi^{3}\left|c \partial_{n} \beta\right|^{2}\left[\partial_{n} \beta\right]_{S}|\psi|^{2} d \sigma d t \\
& +2 s \lambda \int_{t_{0}}^{T} \int_{S} \varphi \partial_{n} \beta_{0}\left|g_{s}\right|^{2} d \sigma d t \\
& =\left|f_{s}\right|_{L^{2}\left(Q^{\prime}\right)}^{2}-2\left(I_{11}+X_{1}+Y_{1}+X_{2}+Y_{2}+I_{21}+X_{3}+I_{31}+I_{32}+I_{33}\right) \text {. }
\end{aligned}
$$

We now consider the surface terms $I_{11}, Y_{1}, Y_{2}$ involving the function $g_{s}$ and write

$$
\left|I_{11}\right|=\left|\int_{t_{0}}^{T} \int_{S} \partial_{t}\left(g_{s}\right) \psi d \sigma d t\right| \leq C s^{-2} \int_{t_{0}}^{T} \int_{S}\left|\partial_{t} g_{s}\right|^{2} d \sigma d t+C s^{2} \int_{t_{0}}^{T} \int_{S}|\psi|^{2} d \sigma d t .
$$

In the proof of Lemma 1.1 we are free to choose $\beta$ such that $\partial_{n} \beta_{1} / c_{0} \leq-1$. Since we assume $c_{0}-c_{1} \geq \Delta$ we obtain

$$
\left[\partial_{n} \beta\right]_{S}=\partial_{n} \beta_{0}-\partial_{n} \beta_{1}=\frac{\partial_{n} \beta_{1}}{c_{0}}\left(c_{1}-c_{0}\right) \geq \Delta>0 .
$$

The second term in (1.14) can thus be absorbed by the term

$$
2 s^{3} \lambda^{3} \int_{t_{0}}^{T} \int_{S} \varphi^{3}\left|c \partial_{n} \beta\right|^{2}\left[\partial_{n} \beta\right]_{S}|\psi|^{2} d \sigma d t
$$

in (1.13) for $s$ sufficiently large. 
The term $Y_{1}$ in (1.12) can be estimated by

$$
\begin{aligned}
& \left|Y_{1}\right|=\left|2 s \lambda \int_{t_{0}}^{T} \int_{S} \varphi c_{1} \partial_{n} \psi_{1} \partial_{n} \beta_{0} g_{s} d \sigma d t\right| \\
& \leq C_{\varepsilon} s \lambda \int_{t_{0}}^{T} \int_{S} \varphi\left|g_{s}\right|^{2} d \sigma d t+\varepsilon s \lambda \int_{t_{0}}^{T} \int_{S} \varphi\left|c_{1} \partial_{n} \psi_{1}\right|^{2}\left(\partial_{n} \beta_{0}\right)^{2} d \sigma d t, \quad \varepsilon>0 .
\end{aligned}
$$

For $\varepsilon$ sufficiently small, the second surface term in (1.16) can be 'absorbed' by the term

$$
2 s \lambda \int_{t_{0}}^{T} \int_{S} \varphi\left[\partial_{n} \beta\right]_{S}\left|c_{1} \partial_{n} \psi_{1}\right|^{2} d \sigma d t
$$

in (1.13) by (1.15).

The term $Y_{2}$ can be estimated by

$$
\begin{aligned}
\left|Y_{2}\right|=\left|2 s \lambda^{2} \int_{t_{0}}^{T} \int_{S} \varphi c_{0}\left(\partial_{n} \beta_{0}\right)^{2} g_{s} \psi d \sigma d t\right| \leq & C s \lambda \int_{t_{0}}^{T} \int_{S} \varphi\left|g_{s}\right|^{2} d \sigma d t \\
& +C s \lambda^{3} \int_{t_{0}}^{T} \int_{S} \varphi c_{0}^{2}\left(\partial_{n} \beta_{0}\right)^{4}|\psi|^{2} d \sigma d t .
\end{aligned}
$$

Observing that $\varphi \leq C T^{4} \varphi^{3}$, the second surface term can be 'absorbed' by the term

$$
2 s^{3} \lambda^{3} \int_{t_{0}}^{T} \int_{S} \varphi^{3}\left|c \partial_{n} \beta\right|^{2}\left[\partial_{n} \beta\right]_{S}|\psi|^{2} d \sigma d t
$$

in (1.13) for $s$ sufficiently large by (1.15). The two previous estimates are the points in the proof where the hypothesis $c_{0}-c_{1} \geq \Delta>0$ is needed.

Note also that

$$
\begin{aligned}
s^{-2}\left|\partial_{t} g_{s}\right|^{2} \leq C s^{-2} e^{-2 s \eta}\left|\partial_{t} g\right|^{2}+C\left(\partial_{t} \eta\right)^{2} e^{-2 s \eta}|g|^{2} & \\
& \leq C s^{-2} e^{-2 s \eta}\left|\partial_{t} g\right|^{2}+C T^{2} \varphi^{4} e^{-2 s \eta}|g|^{2},
\end{aligned}
$$

where we have used that $\left|\partial_{t} \eta\right| \leq C T \varphi^{2}$ [7, equation (90)] (which makes use of the particular choices made above for $K$ and $\bar{\beta}$, which implies that $\bar{\beta} \leq 2 \beta$ ).

Applying the technique presented in the proof of Theorem 3.3 in [7], the previous observations yield the following Carleman estimate (we use the notation $\eta^{(i)}$ 
instead of $\eta$ )

$$
\begin{aligned}
& \quad\left|M_{1}^{(i)}\left(e^{-s \eta^{(i)}} q\right)\right|_{L^{2}\left(Q^{\prime}\right)}^{2}+\left|M_{2}^{(i)}\left(e^{-s \eta^{(i)}} q\right)\right|_{L^{2}\left(Q^{\prime}\right)}^{2} \\
& +s \lambda^{2} \iint_{Q} e^{-2 s \eta^{(i)}} \varphi^{(i)}|\nabla q|^{2} d x d t+s^{3} \lambda^{4} \iint_{Q} e^{-2 s \eta^{(i)}} \varphi^{(i)^{3}}|q|^{2} d x d t \\
& \leq C\left[s \lambda \int_{t_{0}}^{T} \int_{\gamma} e^{-2 s \eta^{(i)}} \varphi^{(i)}\left|\partial_{n} q\right|^{2} d \sigma d t+s^{3} \lambda^{4} \int_{t_{0}}^{T} \int_{\widetilde{B}^{(i)}} e^{-2 s \eta^{(i)}} \varphi^{(i)^{3}}|q|^{2} d x d t\right. \\
& +\iint_{Q^{\prime}} e^{-2 s \eta^{(i)}}\left|\partial_{t} q-\nabla \cdot(c \nabla q)\right|^{2} d x d t+s^{-2} \int_{t_{0}}^{T} \int_{S} e^{-2 s \eta^{(i)}}\left|\partial_{t} g\right|^{2} d \sigma d t \\
& \left.\quad+\int_{t_{0}}^{T} \int_{S} e^{-2 s \eta^{(i)}} \varphi^{(i)^{4}}|g|^{2} d \sigma d t+s \lambda \int_{t_{0}}^{T} \int_{S} e^{-2 s \eta^{(i)}} \varphi^{(i)}|g|^{2} d \sigma d t\right],
\end{aligned}
$$

for $i=1,2$, and for $\lambda \geq \lambda_{0}\left(\Omega, \gamma, \mathcal{O}^{(1)}, \mathcal{O}^{(2)}, c_{\text {min }}, c_{\text {max }}, \Delta\right)$ and $s \geq s_{0}\left(\lambda_{0}\right)$ (the sets $\widetilde{B}^{(i)}$ were introduced in Lemma 1.1). Note that the condition $[c]_{s} \geq 0$ is needed to obtain the previous estimate.

Adding (1.17) for $i=1,2$, we deduce (1.5) with the same argumentation as in the proof of Theorem 3.4 in [7]. The terms integrated over $\left(t_{0}, T\right) \times \widetilde{B}^{(i)}$ are absorbed by other terms using properties (1.1)-(1.3) of $\widetilde{\beta}^{(i)}, i=1,2$.

Remark 1.3. The Carleman estimate that was just derived is peculiar because of the presence of terms integrated on the interface $S$. In particular, two terms involve the function $g$ with different powers for the parameters $s$ and $\lambda$ and for the weight functions $\varphi^{(i)}, i=1,2$. This Carleman estimate is the key ingredient in the subsequent analysis. The interface terms will require some special treatment. The two parameters $s$ and $\lambda$ will also have an important role to play in the next section.

Remark 1.4. In the case $g=0$, the previous Carleman estimate simplifies. By inspection of the proof of Theorem 1.2, observe that in the case $g=0$, the condition $c_{\left.0\right|_{S}}-c_{\left.1\right|_{S}} \geq 0$ is sufficient to obtain the Carleman estimate [7]. The case of $c_{0||_{S}}-c_{\left.1\right|_{S}}<0$ remains open in the case of a dimension greater than equal to 2 (in the one-dimensional case a Carleman estimate for the heat operator, $\partial_{t} \pm \partial_{x}\left(c \partial_{x}\right)$, in arbitrary situations, can be found in $\left.[3,4]\right)$.

\section{Uniqueness and stability estimate for the dif- fusion coefficients}

In this section we establish a uniqueness result for the discontinuous diffusion coefficient $c$ as well as a stability inequality. This inequality estimates the discrepancy in the coefficients $c$ and $\widetilde{c}$ of two materials (with the same geometry) with an upper bound given by some Sobolev norms of the difference between 
the solutions $y$ and $\widetilde{y}$ to

$$
\begin{cases}\partial_{t} \widetilde{y}-\nabla \cdot(\widetilde{c} \nabla \widetilde{y})=0, & \text { in }(0, T) \times \Omega, \\ \widetilde{y}(t, x)=h(t, x), & \text { on }(0, T) \times \Gamma, \\ \text { transmission conditions }(\widetilde{\mathrm{TC} 1}), & \text { on }[0, T] \times S, \\ \widetilde{y}(0)=\widetilde{y}_{0}, & \end{cases}
$$

with

$(\widetilde{\mathrm{TC} 1}) \quad \widetilde{y}_{\mid[0, T] \times S_{0}}=\widetilde{y}_{\mid[0, T] \times S_{1}}, \quad \widetilde{c}_{0} \partial_{n} \widetilde{y}_{\mid[0, T] \times S_{0}}=\widetilde{c}_{1} \partial_{n} \widetilde{y}_{\mid[0, T] \times S_{1}}$,

and

$$
\begin{cases}\partial_{t} y-\nabla \cdot(c \nabla y)=0, & \text { in }(0, T) \times \Omega, \\ y(t, x)=h(t, x), & \text { on }(0, T) \times \Gamma, \\ \text { transmission conditions }(\mathrm{TC} 1), & \text { on }[0, T] \times S, \\ y(0)=y_{0} . & \end{cases}
$$

The Carleman estimate proved in the previous section will be the key ingredient in the proof of such a stability estimate.

We introduce

$$
\xi=c-\widetilde{c}= \begin{cases}\xi_{0}=c_{0}-\widetilde{c}_{0} & \text { in } \Omega_{0}, \\ \xi_{1}=c_{1}-\widetilde{c}_{1} & \text { in } \Omega_{1} .\end{cases}
$$

We set $u=y-\widetilde{y}$ and $v=\partial_{t} u$. Then $v$ is solution to the following problem

$$
\begin{cases}\partial_{t} v-\nabla \cdot(c \nabla v)=\nabla \cdot\left(\xi \nabla \partial_{t} \widetilde{y}\right), & \text { in }(0, T) \times \Omega^{\prime}, \\ v=0, & \text { on }(0, T) \times \Gamma, \\ \text { transmission conditions }(\mathrm{TC} 2), & \text { on }[0, T] \times S,\end{cases}
$$

with

$$
\left\{\begin{array}{l}
v_{\left.\right|_{[0, T] \times S_{0}}}=v_{\left.\right|_{[0, T] \times S_{1}}} \\
c_{0} \partial_{n} v_{\left.\right|_{[0, T] \times S_{0}}}=c_{1} \partial_{n} v_{\left.\right|_{[0, T] \times S_{1}}}+g(t, x)
\end{array}\right.
$$

where

$$
g(t, x)=\xi_{1} \partial_{n} \partial_{t} \widetilde{y}_{\mid[0, T] \times S_{1}}-\xi_{0} \partial_{n} \partial_{t} \widetilde{y}_{\mid[0, T] \times S_{0}}=\alpha \partial_{n} \partial_{t} \widetilde{y}_{\mid[0, T] \times S_{0}},
$$

with $\alpha=\xi_{\left.1\right|_{S_{1}}} \frac{\widetilde{c}_{0_{\left.\right|_{S_{0}}}}}{\widetilde{c}_{\left.\right|_{S_{1}}}}-\xi_{\left.0\right|_{S_{0}}}$.

Let $T^{\prime}=\frac{1}{2}\left(T+t_{0}\right)$. We make the following assumption.

Assumption 2.1. The solutions $\widetilde{y}$ and $y$ belong to $H^{2}\left(t_{0}, T, H^{1}(\Omega)\right)$ and are such that $y_{\left.\right|_{\Omega_{i}}} \in H^{1}\left(t_{0}, T, H^{2}\left(\Omega_{i}\right)\right), \widetilde{y}_{\left.\right|_{\Omega_{i}}} \in H^{2}\left(t_{0}, T, H^{2}\left(\Omega_{i}\right)\right), i=0,1$. Furthermore, $\widetilde{y}$ satisfies

1. Let $r>0$. The solution $\widetilde{y}$ is such that $\left|\Delta \widetilde{y}\left(T^{\prime}\right)\right| \geq r>0$ in $\Omega^{\prime}$; 
2. $\widetilde{y}_{\mid \Omega_{i}}$ is in a bounded domain of $W^{2, \infty}\left(t_{0}, T, H^{2}\left(\Omega_{i}\right)\right), i=0,1$ : there exists $M>0$ such that

$$
\begin{aligned}
& \quad\left|\widetilde{y}_{\mid \Omega_{i}}(t, .)\right|_{H^{2}\left(\Omega_{i}\right)}^{2}+\left|\partial_{t} \widetilde{y}_{\mid \Omega_{i}}(t, .)\right|_{H^{2}\left(\Omega_{i}\right)}^{2}+\left|\partial_{t}^{2} \widetilde{y}_{\Omega_{\Omega_{i}}}(t, .)\right|_{H^{2}\left(\Omega_{i}\right)}^{2} \leq M, i=0,1, \\
& \text { a.e. for } t \in\left(t_{0}, T\right) ;
\end{aligned}
$$

3. $\Delta \partial_{t} \widetilde{y}_{\Omega_{i}}$ is in a bounded domain of $L^{2}\left(t_{0}, T, L^{\infty}\left(\Omega_{i}\right)\right), i=0,1$ : there exists $K>0$ such that

$$
\int_{t_{0}}^{T}\left|\Delta \partial_{t} \widetilde{y}_{\Omega_{i}}(t, .)\right|_{L^{\infty}\left(\Omega_{i}\right)}^{2} d t \leq K^{2}, i=0,1 .
$$

In Section 3 we shall show that for any initial conditions $y_{0}, \widetilde{y}_{0}$ in $L^{2}(\Omega)$ we can achieve the properties listed in Assumption 2.1 by using some particular boundary conditions $h(t, x)$.

From Assumption 2.1, the functions $\widetilde{y}$ and $v$ are such that $\widetilde{y}_{\mid \Omega_{i}}, v_{\left.\right|_{\Omega_{i}}} \in H^{2}\left(\Omega_{i}\right)$, $i=0,1$. Then $g \in H^{1}\left(t_{0}, T, H^{\frac{1}{2}}(S)\right)$. The second equality in condition transmission (TC2) thus takes place in the space $H^{\frac{1}{2}}(S)$. Observe that $v=\partial_{t}(y-\widetilde{y}) \in \aleph_{g}$ from the above assumption. We can thus apply Carleman estimate (1.5) to $v$.

We shall use the notations of the proof of Theorem 1.2. We set $\psi^{(i)}=e^{-s \eta^{(i)}} v$, $i=1,2$. With the operator $M_{2}^{(i)}$ defined in (1.10) we introduce, following [2],

$$
I^{(i)}=\int_{t_{0}}^{T^{\prime}} \int_{\Omega^{\prime}} M_{2}^{(i)} \psi^{(i)} \varphi^{(i)^{\frac{3}{2}}} \psi^{(i)} d x d t, \quad i=1,2, \quad \text { and } I=\frac{1}{2}\left(I^{(1)}+I^{(2)}\right) .
$$

Note the additional $\varphi^{(i)^{\frac{3}{2}}}$ factor as compared to [2]. This will be of importance below.

We have the following estimates.

Lemma 2.2. Let $\lambda \geq \lambda_{1}$ and $s \geq s_{1}$ then

$$
\begin{aligned}
|I| \leq C s^{-3 / 2} \lambda^{-2}\left[s \lambda \int_{t_{0}}^{T} \int_{\gamma}\left(e^{-2 s \eta^{(1)}} \varphi^{(1)}+e^{-2 s \eta^{(2)}} \varphi^{(2)}\right)\left|\partial_{n} v\right|^{2} d \sigma d t\right. \\
+\iint_{Q^{\prime}}\left(e^{-2 s \eta^{(1)}}+e^{-2 s \eta^{(2)}}\right)\left|\partial_{t} v-\nabla \cdot(c \nabla v)\right|^{2} d x d t \\
+s \lambda \int_{t_{0}}^{T} \int_{S}\left(e^{-2 s \eta^{(1)}} \varphi^{(1)}+e^{-2 s \eta^{(2)}} \varphi^{(2)}\right)|g|^{2} d \sigma d t \\
+\int_{t_{0}}^{T} \int_{S}\left(e^{-2 s \eta^{(1)}} \varphi^{(1)^{4}}+e^{-2 s \eta^{(2)}} \varphi^{(2)^{4}}\right)|g|^{2} d \sigma d t \\
\left.+s^{-2} \int_{t_{0}}^{T} \int_{S}\left(e^{-2 s \eta^{(1)}}+e^{-2 s \eta^{(2)}}\right)\left|\partial_{t} g\right|^{2} d \sigma d t\right] .
\end{aligned}
$$

Proof. Observe that

$$
\begin{array}{r}
\left|I^{(i)}\right| \leq \frac{1}{2} s^{-3 / 2} \lambda^{-2}\left(\left|M_{2}^{(i)} \psi^{(i)}\right|_{L^{2}\left(Q^{\prime}\right)}^{2}+s^{3} \lambda^{4} \iint_{Q} \varphi^{(i)^{3}} e^{-2 s \eta^{(i)}}|v|^{2} d x d t\right), \\
i=1,2 .
\end{array}
$$


(We recall that $Q^{\prime}=\left(t_{0}, T\right) \times \Omega^{\prime}$.) Thus

$$
\begin{aligned}
|I| \leq \frac{1}{2} s^{-3 / 2} \lambda^{-2}(\mid & \left.M_{2}^{(1)} \psi^{(1)}\right|_{L^{2}\left(Q^{\prime}\right)} ^{2}+\left|M_{2}^{(2)} \psi^{(2)}\right|_{L^{2}\left(Q^{\prime}\right)}^{2} \\
& \left.+s^{3} \lambda^{4} \iint_{Q}\left(e^{-2 s \eta^{(1)}} \varphi^{(1)^{3}}+e^{-2 s \eta^{(2)}} \varphi^{(2)^{3}}\right)|v|^{2} d x d t\right),
\end{aligned}
$$

which yields the result from Carleman estimate (1.5).

Lemma 2.3. Let $\lambda \geq \lambda_{1}$ and $s \geq s_{1}$ then

$$
\begin{aligned}
\int_{\Omega^{\prime}}\left(e^{-2 s \eta^{(1)}} \varphi^{(1)^{\frac{3}{2}}}+e^{-2 s \eta^{(2)}} \varphi^{(2)^{\frac{3}{2}}}\right)\left(T^{\prime}, x\right)\left|v\left(T^{\prime}, .\right)\right|^{2} d x \\
\leq C s^{-3 / 2} \lambda^{-2}\left[s \lambda \int_{t_{0}}^{T} \int_{\gamma}\left(e^{-2 s \eta^{(1)}} \varphi^{(1)}+e^{-2 s \eta^{(2)}} \varphi^{(2)}\right)\left|\partial_{n} v\right|^{2} d \sigma d t\right. \\
+\iint_{Q^{\prime}}\left(e^{-2 s \eta^{(1)}}+e^{-2 s \eta^{(2)}}\right)\left|\nabla \cdot\left(\xi \nabla \partial_{t} \widetilde{y}\right)\right|^{2} d x d t \\
+s \lambda \int_{t_{0}}^{T} \int_{S}\left(e^{-2 s \eta^{(1)}} \varphi^{(1)}+e^{-2 s \eta^{(2)}} \varphi^{(2)}\right)|g|^{2} d \sigma d t \\
+\int_{t_{0}}^{T} \int_{S}\left(e^{-2 s \eta^{(1)}} \varphi^{(1)^{4}}+e^{-2 s \eta^{(2)}} \varphi^{(2)^{4}}\right)|g|^{2} d \sigma d t \\
\left.+s^{-2} \int_{t_{0}}^{T} \int_{S}\left(e^{-2 s \eta^{(1)}}+e^{-2 s \eta^{(2)}}\right)\left|\partial_{t} g\right|^{2} d \sigma d t\right]
\end{aligned}
$$

Proof. We evaluate integral $I^{(i)}, i=1,2$, using (1.10)

$$
\begin{aligned}
& I^{(i)}=\int_{t_{0}}^{T_{\Omega^{\prime}}^{\prime}} \int_{t}\left(\partial_{t} \psi^{(i)}-2 s \lambda \varphi^{(i)} c \nabla \beta^{(i)} \cdot \nabla \psi^{(i)}\right. \\
&\left.-2 s \lambda^{2} \varphi^{(i)} c\left|\nabla \beta^{(i)}\right|^{2} \psi^{(i)}\right) \varphi^{(i)^{\frac{3}{2}}} \psi^{(i)} d x d t \\
&= \frac{1}{2} \int_{t_{0}}^{T^{\prime}} \int_{\Omega^{\prime}} \varphi^{(i) \frac{3}{2}} \partial_{t}\left|\psi^{(i)}\right|^{2} d x d t-s \lambda \int_{t_{0}}^{T^{\prime}} \int_{\Omega^{\prime}} \varphi^{(i)^{\frac{5}{2}}} c \nabla \beta^{(i)} \cdot \nabla\left|\psi^{(i)}\right|^{2} d x d t \\
&-2 s \lambda^{2} \int_{t_{0}}^{T^{\prime}} \int_{\Omega^{\prime}} \varphi^{(i)^{\frac{5}{2}}} c\left|\nabla \beta^{(i)}\right|^{2}\left|\psi^{(i)}\right|^{2} d x d t \\
&= \frac{1}{2} \int_{t_{0}}^{T_{\Omega^{\prime}}^{\prime}} \varphi^{(i)^{\frac{3}{2}}} \partial_{t}\left|\psi^{(i)}\right|^{2} d x d t+s \lambda \int_{t_{0}}^{T^{\prime}} \int_{\Omega^{\prime}} \nabla \cdot\left(\varphi^{(i)^{\frac{5}{2}}} c \nabla \beta^{(i)}\right)\left|\psi^{(i)}\right|^{2} d x d t \\
&-2 s \lambda^{2} \int_{t_{0}}^{T^{\prime}} \int_{\Omega^{\prime}} \varphi^{(i)^{\frac{5}{2}}} c\left|\nabla \beta^{(i)}\right|^{2}\left|\psi^{(i)}\right|^{2} d x d t
\end{aligned}
$$

by integration by parts, without any remaining integral over $\left(t_{0}, T^{\prime}\right) \times S$ by condition transmission (1.8). With an integration by parts w.r.t. $t$ in the first 
integral, we then obtain

$$
\begin{array}{r}
\text { (2.4) } \frac{1}{2} \int_{\Omega^{\prime}} \varphi^{(i)^{\frac{3}{2}}}\left|\psi^{(i)}\left(T^{\prime}, .\right)\right|^{2} d x=I^{(i)}+\frac{1}{2} s \lambda^{2} \int_{t_{0}}^{T^{\prime}} \int_{\Omega^{\prime}} \varphi^{(i)^{\frac{5}{2}}} c\left|\nabla \beta^{(i)}\right|^{2}\left|\psi^{(i)}\right|^{2} d x d t \\
-s \lambda \int_{t_{0}}^{T_{\Omega^{\prime}}^{\prime}} \varphi^{(i)^{\frac{5}{2}}} \nabla \cdot\left(c \nabla \beta^{(i)}\right)\left|\psi^{(i)}\right|^{2} d x d t+\frac{3}{4} \int_{t_{0}}^{T^{\prime}} \int_{\Omega^{\prime}}\left(\partial_{t} \varphi^{(i)}\right) \varphi^{(i)^{\frac{1}{2}}}\left|\psi^{(i)}\right|^{2} d x d t, \\
i=1,2,
\end{array}
$$

since $\varphi^{(i) \frac{3}{2}} \psi^{(i)}\left(t_{0},.\right)=0$. Adding $(2.4)$ for $i=1,2$ we obtain

$$
\begin{gathered}
\int_{\Omega^{\prime}}\left(e^{-2 s \eta^{(1)}} \varphi^{(1)^{\frac{3}{2}}}+e^{-2 s \eta^{(2)}} \varphi^{(2)^{\frac{3}{2}}}\right)\left(T^{\prime}, x\right)\left|v\left(T^{\prime}, .\right)\right|^{2} d x \leq 4|I| \\
+C\left(s \lambda^{2}+s \lambda+1\right) \int_{t_{0}}^{T^{\prime}} \int_{\Omega^{\prime}}\left(e^{-2 s \eta^{(1)}(t, x)} \varphi^{(1)^{\frac{5}{2}}}+e^{-2 s \eta^{(2)}(t, x)} \varphi^{(2)^{\frac{5}{2}}}\right)|v|^{2} d x d t,
\end{gathered}
$$

observing that $\left|\partial_{t} \varphi^{(i)}\right| \leq C T \varphi^{(i)^{2}}, i=1,2$. We use Carleman estimate (1.5) to obtain an upper-bound for the last term in (2.5) which yields the result by Lemma 2.2 .

We shall now assume:

Assumption 2.4. The diffusion coefficients $c$ and $\widetilde{c}$ are piecewise constant, in the sense that $c_{\left.\right|_{\Omega_{i}}}$, resp. $\widetilde{c}_{\left.\right|_{\Omega_{i}}}$, are constant in each connected component of $\Omega_{i}$, $i=0,1$. We define

$$
\begin{aligned}
& c_{0, j}=c_{\left.\right|_{\Omega_{0, j}}}, j=1, \ldots, p_{0}, \\
& c_{1, j}=c_{\left.\right|_{\Omega_{1, j}}}, j=1, \ldots, p_{1},
\end{aligned}
$$

with similar notations for $\widetilde{c}$ and $\xi$.

In this case observe that, in $\Omega^{\prime}$,

$$
\begin{aligned}
v\left(T^{\prime}, x\right)=c \Delta & u\left(T^{\prime}, x\right)+\xi \Delta \widetilde{y}\left(T^{\prime}, x\right) \\
=\sum_{j=1}^{p_{0}} c_{0, j} \Delta & u\left(T^{\prime}, x\right) \chi_{\Omega_{0, j}}+\sum_{j=1}^{p_{1}} c_{1, j} \Delta u\left(T^{\prime}, x\right) \chi_{\Omega_{1, j}} \\
& \quad+\sum_{j=1}^{p_{0}} \xi_{0, j} \Delta \widetilde{y}\left(T^{\prime}, x\right) \chi_{\Omega_{0, j}}+\sum_{j=1}^{p_{1}} \xi_{1, j} \Delta \widetilde{y}\left(T^{\prime}, x\right) \chi_{\Omega_{1, j}},
\end{aligned}
$$

from the equation satisfied by $u$ expressed at time $T^{\prime}$ and the definition of $v$ above. 
From Lemma 2.3, we obtain

$$
\begin{gathered}
\int_{\Omega^{\prime}}\left(e^{-2 s \eta^{(1)}} \varphi^{(1)^{\frac{3}{2}}}+e^{-2 s \eta^{(2)}} \varphi^{(2)^{\frac{3}{2}}}\right)\left(T^{\prime}, x\right)\left|\xi \Delta \widetilde{y}\left(T^{\prime}, x\right)\right|^{2} d x \\
\leq C \int_{\Omega^{\prime}}\left(e^{-2 s \eta^{(1)}} \varphi^{(1)^{\frac{3}{2}}}+e^{-2 s \eta^{(2)}} \varphi^{(2)^{\frac{3}{2}}}\right)\left(T^{\prime}, x\right)\left|c \Delta u\left(T^{\prime}, x\right)\right|^{2} d x \\
+C s^{-3 / 2} \lambda^{-2}\left[s \lambda \int_{t_{0}}^{T} \int_{\gamma}\left(e^{-2 s \eta^{(1)}} \varphi^{(1)}+e^{-2 s \eta^{(2)}} \varphi^{(2)}\right)\left|\partial_{n} v\right|^{2} d \sigma d t\right. \\
+\iint_{Q^{\prime}}\left(e^{-2 s \eta^{(1)}}+e^{-2 s \eta^{(2)}}\right)\left|\nabla \cdot\left(\xi \nabla \partial_{t} \widetilde{y}\right)\right|^{2} d x d t \\
+s \lambda \int_{t_{0}}^{T} \int_{S}\left(e^{-2 s \eta^{(1)}} \varphi^{(1)}+e^{-2 s \eta^{(2)}} \varphi^{(2)}\right)|g|^{2} d \sigma d t \\
+\int_{t_{0}}^{T} \int_{S}\left(e^{-2 s \eta^{(1)}} \varphi^{(1)^{4}}+e^{-2 s \eta^{(2)}} \varphi^{\left.(2)^{4}\right)}|g|^{2} d \sigma d t\right. \\
\left.+s^{-2} \int_{t_{0}}^{T} \int_{S}\left(e^{-2 s \eta^{(1)}}+e^{-2 s \eta^{(2)}}\right)\left|\partial_{t} g\right|^{2} d \sigma d t\right] .
\end{gathered}
$$

From Assumption 2.1 we find that

$$
\left|\Delta \partial_{t} \widetilde{y}(t, x)\right|^{2} \leq k^{2}(t)\left|\Delta \widetilde{y}\left(T^{\prime}, x\right)\right|^{2}, \text { in each }\left(t_{0}, T\right) \times \Omega_{i}, i=0,1,
$$

for

$$
k(t)=\frac{1}{r} \sup _{i=0,1}\left|\Delta \partial_{t} \widetilde{y}_{\left.\right|_{\Omega_{i}}}(t, .)\right|_{L^{\infty}\left(\Omega_{i}\right)}
$$

From Assumption 2.1, $k \in L^{2}\left(t_{0}, T\right)$ and $|k|_{L^{2}\left(t_{0}, T\right)} \leq K^{\prime}=\frac{1}{r} K$. These observations yield the following estimation of the third term in the r.h.s. of (2.6):

$$
\begin{aligned}
& \int_{t_{0}}^{T} \int_{\Omega_{i}}\left(e^{-2 s \eta^{(1)}}+e^{-2 s \eta^{(2)}}\right)\left|\xi \Delta \partial_{t} \widetilde{y}\right|^{2} d x d t \\
\leq & K^{\prime 2} \sum_{i=0,1} \sum_{j=1}^{p_{i}}\left|\xi_{i, j}\right|^{2} \int_{\Omega_{i, j}}\left(e^{-2 s \eta^{(1)}\left(T^{\prime}, x\right)}+e^{-2 s \eta^{(2)}\left(T^{\prime}, x\right)}\right)\left|\Delta \widetilde{y}\left(T^{\prime}, x\right)\right|^{2} d x,
\end{aligned}
$$

where we have used that

$$
e^{-2 s \eta^{(i)}(t, x)} \leq e^{-2 s \eta^{(i)}\left(T^{\prime}, x\right)}, \quad x \in \Omega, t \in\left(t_{0}, T\right), i=1,2 .
$$

Observing that $0<C \leq \varphi^{(i)}, i=1,2$ since $\beta^{(i)} \geq 0$ and $\frac{1}{(T-t)\left(t-t_{0}\right)} \geq C>0$, we obtain

$$
\begin{aligned}
& \int_{t_{0}}^{T} \int_{\Omega_{i}}\left(e^{-2 s \eta^{(1)}}+\right.\left.e^{-2 s \eta^{(2)}}\right)\left|\xi \Delta \partial_{t} \widetilde{y}\right|^{2} d x d t \\
& \leq K^{\prime 2} \sum_{i=0,1} \sum_{j=1}^{p_{i}}\left|\xi_{i, j}\right|^{2} \int_{\Omega_{i, j}}\left(e^{-2 s \eta^{(1)}} \varphi^{(1)^{\frac{3}{2}}}\right. \\
& \\
&\left.\quad+e^{-2 s \eta^{(2)}\left(T^{\prime}, x\right)} \varphi^{(2)^{\frac{3}{2}}}\right)\left(T^{\prime}, x\right)\left|\Delta \widetilde{y}\left(T^{\prime}, x\right)\right|^{2} d x .
\end{aligned}
$$


We now treat the interface terms that appear in the r.h.s. of the Carleman estimate. Recall that

$$
\begin{aligned}
& g(t, x)=\alpha \partial_{n} \partial_{t} \widetilde{y}_{\mid[0, T] \times S_{0}}, \\
& \alpha=\xi_{1} \frac{\widetilde{c}_{0}}{\widetilde{c}_{1}}-\xi_{0} .
\end{aligned}
$$

Note that $\eta(t,$.$) is constant on S$. We denote this constant by $\eta(t, S)$. More generally we shall denote $\rho(S)$ the value on $S$ of a function $\rho$ which is constant on $S$. We obtain

$$
\begin{aligned}
\int_{t_{0}}^{T} \int_{S}\left(e^{-2 s \eta^{(1)}}\right. & \left.+e^{-2 s \eta^{(2)}}\right)\left|\partial_{t} g\right|^{2} d \sigma d t \\
\leq \int_{t_{0}}^{T}\left(e^{-2 s \eta^{(1)}(t, S)}\right. & \left.+e^{-2 s \eta^{(2)}(t, S)}\right) \int_{S}\left|\partial_{t} g\right|^{2} d \sigma d t \\
& \leq M^{\prime}|\xi|^{2} \int_{t_{0}}^{T}\left(e^{-2 s \eta^{(1)}(t, S)}+e^{-2 s \eta^{(2)}(t, S)}\right) d t,
\end{aligned}
$$

from trace inequalities and from Assumption 2.1, for $M^{\prime}=C_{T r}\left(1+\frac{c_{\max }}{c_{\min }}\right)^{2} M$, where $|\xi|=\sqrt{\xi_{0}^{2}+\xi_{1}^{2}}$, since $|\alpha| \leq\left(1+\frac{c_{\max }}{c_{\min }}\right)|\xi|$ from Assumption 0.2. The constant $C_{T r}$ is the constant found in the trace estimates

$$
\int_{S}\left|\partial_{n} \widetilde{\rho}\right|^{2} \leq C_{T r}|\rho|_{H^{2}\left(\Omega_{i}\right)}^{2}, \quad i=0,1,
$$

if $\rho_{\mid \Omega_{i}} \in H^{2}\left(\Omega_{i}\right), i=0,1$. Similarly, since $\varphi^{(i)}, i=1,2$, are constant on $S$, we have

$$
\begin{aligned}
\int_{t_{0}}^{T} \int_{S}\left(e^{-2 s \eta^{(1)}} \varphi^{(1)^{j}}+e^{-2 s \eta^{(2)}} \varphi^{(2)^{j}}\right)|g|^{2} d \sigma d t & \\
& \leq M^{\prime}|\xi|^{2} \int_{t_{0}}^{T}\left(e^{-2 s \eta^{(1)}} \varphi^{(1)^{j}}+e^{-2 s \eta^{(2)}} \varphi^{(2)^{j}}\right)(t, S) d t, \quad j \in \mathbb{N} .
\end{aligned}
$$

With

$$
w_{k}(s, \lambda):=\int_{t_{0}}^{T}\left(e^{-2 s \eta^{(1)}} \varphi^{(1)^{k}}+e^{-2 s \eta^{(2)}} \varphi^{(2)^{k}}\right)(t, S) d t, \quad k \in \mathbb{N},
$$

and

$$
\begin{array}{r}
W_{i, j}(s, \lambda):=\int_{\Omega_{i, j}}\left(e^{-2 s \eta^{(1)}} \varphi^{(1)^{\frac{3}{2}}}+e^{-2 s \eta^{(2)}} \varphi^{(2)^{\frac{3}{2}}}\right)\left(T^{\prime}, x\right)\left|\Delta \widetilde{y}\left(T^{\prime}, x\right)\right|^{2} d x, \\
i=0,1, j=1, \ldots, p_{i},
\end{array}
$$


we thus obtain, for $\lambda \geq \lambda_{1}$ and $s \geq s_{1}$,

$$
\begin{aligned}
& \sum_{i=0,1} \sum_{j=1}^{p_{i}}\left|\xi_{i, j}\right|^{2}\left\{\left(1-C K^{\prime 2} s^{-\frac{3}{2}} \lambda^{-2}\right) W_{i, j}(s, \lambda)\right. \\
& \left.-C M^{\prime}\left[s^{-\frac{1}{2}} \lambda^{-1} w_{1}(s, \lambda)+s^{-\frac{3}{2}} \lambda^{-2} w_{4}(s, \lambda)+s^{-\frac{7}{2}} \lambda^{-2} w_{0}(s, \lambda)\right]\right\} \\
& \leq C \int_{\Omega^{\prime}}\left(e^{-2 s \eta^{(1)}} \varphi^{(1)^{\frac{3}{2}}}+e^{-2 s \eta^{(2)}} \varphi^{(2)^{\frac{3}{2}}}\right)\left(T^{\prime}, x\right)\left|c \Delta u\left(T^{\prime}, .\right)\right|^{2} d x \\
& \quad+C s^{-\frac{1}{2}} \lambda^{-1} \int_{t_{0}}^{T} \int_{\gamma}\left(e^{-2 s \eta^{(1)}} \varphi^{(1)}+e^{-2 s \eta^{(2)}} \varphi^{(2)}\right)\left|\partial_{n} v\right|^{2} d \sigma d t .
\end{aligned}
$$

To obtain a stability result we need to prove that the coefficients for $\left|\xi_{i, j}\right|^{2}$, $i=0,1, j=1, \ldots, p_{i}$, can be made positive. To do so we need to understand the behavior of the integrals $w_{k}(s, \lambda)$ and $W_{i, j}(s, \lambda)$ as $s$ and $\lambda$ become large.

We first establish the asymptotic behavior of $w_{k}(s, \lambda)$. We set

$$
w_{k}^{(i)}(s, \lambda):=\int_{t_{0}}^{T} e^{-2 s \eta^{(i)}(t, S)} \varphi^{(i)}(t, S) d t, \quad k \in \mathbb{N} .
$$

Lemma 2.5. The following estimates holds

$$
\begin{aligned}
w_{k}^{(i)}(s, \lambda)=e^{-2 s \eta^{(i)}\left(T^{\prime}, S\right)} \varphi^{(i)^{k}}\left(T^{\prime}, S\right)\left\{\frac{\sqrt{\pi} s^{-\frac{1}{2}}}{\sqrt{\phi^{\prime \prime}\left(T^{\prime}\right)} \sqrt{e^{\lambda \bar{\beta}}-e^{\lambda \beta^{(i)}(S)}}}\right. \\
\left.+\mathcal{O}\left(\frac{s^{-\frac{3}{2}}}{\left(e^{\lambda \bar{\beta}}-e^{\lambda \beta^{(i)}(S)}\right)^{\frac{3}{2}}}\right)\right\},
\end{aligned}
$$

with $\phi(t)=\frac{1}{(T-t)\left(t-t_{0}\right)}$, for $s>s_{2}>0$ and $\lambda>\lambda_{2}>0$.

Proof. Let $T^{(2)}$ and $T^{(3)}$ be such that $t_{0}<T^{(2)}<T^{\prime}<T^{(3)}<T$. We choose $\chi_{1} \in \mathscr{C}_{c}^{\infty}\left(\left[t_{0}, T^{(2)}\right)\right), \chi_{2} \in \mathscr{C}_{c}^{\infty}\left(\left(t_{0}, T\right)\right)$, and $\chi_{3} \in \mathscr{C}_{c}^{\infty}\left(\left(T^{(3)}, T\right]\right)$, all three nonnegative, such that $\chi_{1}+\chi_{2}+\chi_{3}=1$ and $\chi_{1}=1$ in a neighborhood of $t_{0}, \chi_{2}=1$ in a neighborhood of $T^{\prime}$, and $\chi_{3}=1$ in a neighborhood of $T$. With this partition of unity we break $w_{k}^{(i)}$ into three pieces: $w_{k}^{(i)}=w_{k}^{(i, 1)}+w_{k}^{(i, 2)}+w_{k}^{(i, 3)}$ with

$$
w_{k}^{(i, j)}(s, \lambda):=\int_{t_{0}}^{T} e^{-2 s \eta^{(i)}(t, S)} \varphi^{(i)^{k}}(t, S) \chi_{j}(t) d t, \quad j=1,2,3 .
$$

The first and the third term are treated similarly. Let $s_{2}>0$ and $\lambda_{2}>0$. We set $\tau(s, \lambda, S)=s\left(e^{\lambda \bar{\beta}}-e^{\lambda \beta^{(i)}(S)}\right)$. We observe

$$
\begin{aligned}
w_{k}^{(i, 1)}(s, \lambda) & =e^{k \lambda \beta^{(i)}(S)} \int_{t_{0}}^{T} e^{-2 s \eta^{(i)}(t, S)} \phi^{k}(t) \chi_{1}(t) d t \\
& \leq e^{k \lambda \beta^{(i)}(S)} e^{-2\left(s-s_{2}\right) \eta^{(i)}\left(T^{(2)}, S\right)} \int_{t_{0}}^{T} e^{-2 s_{2} \eta^{(i)}(t, S)} \phi^{k}(t) \chi_{1}(t) d t \\
& \leq C\left(s_{2}, \lambda_{2}\right) e^{k \lambda \beta^{(i)}(S)} e^{-2\left(s-s_{2}\right) \eta^{(i)}\left(T^{(2)}, S\right)} \\
& \leq C e^{k \lambda \beta^{(i)}(S)} e^{-2 s \eta^{(i)}\left(T^{\prime}, S\right)} e^{-2 s\left(\eta^{(i)}\left(T^{(2)}, S\right)-\eta^{(i)}\left(T^{\prime}, S\right)\right)} \\
& =e^{k \lambda \beta^{(i)}(S)} e^{-2 s \eta^{(i)}\left(T^{\prime}, S\right)} \mathcal{O}\left(\tau(s, \lambda, S)^{-l}\right),
\end{aligned}
$$


for all $l \in \mathbb{N}$, if $s>s_{2}$ and $\lambda>\lambda_{2}$, since

$$
\left.s\left(\eta^{(i)}\left(T^{(2)}, S\right)-\eta^{(i)}\left(T^{\prime}, S\right)\right)\right)=\tau(s, \lambda, S)\left(\phi\left(T^{(2)}\right)-\phi\left(T^{\prime}\right)\right),
$$

and $\phi\left(T^{(2)}\right)-\phi\left(T^{\prime}\right)>0$.

For the second term $w_{k}^{(i, 2)}$ we write

$$
\begin{aligned}
w_{k}^{(i, 2)}(s, \lambda) & :=e^{k \lambda \beta^{(i)}(S)} \int_{t_{0}}^{T} e^{-2 \tau(s, \lambda, S) \phi(t)} \phi^{k}(t) \chi_{2}(t) d t \\
& =e^{k \lambda \beta^{(i)}(S)} e^{-2 \tau(s, \lambda, S) \phi\left(T^{\prime}\right)} \int_{t_{0}}^{T} e^{-2 \tau(s, \lambda, S)\left(\phi(t)-\phi\left(T^{\prime}\right)\right)} \phi^{k}(t) \chi_{2}(t) d t .
\end{aligned}
$$

We then apply the following stationary phase formula [15, Theorem 7.7.5], in one dimension,

$$
\begin{aligned}
& \left|\int u(y) e^{i \omega f(y)} d y-e^{i \omega f\left(y_{0}\right)}\left(\frac{\omega f^{\prime \prime}\left(y_{0}\right)}{2 \pi i}\right)^{-\frac{1}{2}} \sum_{l<l_{1}} \omega^{-l} L_{l} u\right| \\
& \quad \leq C \omega^{-l_{1}-\frac{1}{2}} \sum_{|\alpha| \leq 2 l_{1}} \sup \left|\partial^{\alpha} u\right|, \omega>0, u \in \mathscr{C}_{c}^{\infty},
\end{aligned}
$$

where $L_{l}$ is a differential operator of order $2 l$ evaluated at $T^{\prime}, L_{0} u=u\left(T^{\prime}\right)$. (To obtain the additional factor $-\frac{1}{2}$ in the r.h.s. as compared to the formula given in [15, Theorem 7.7.5], simply write the formula to the order $l_{1}+1$.), This formula is valid if $\operatorname{Im}(f) \geq 0, \operatorname{Im}\left(f\left(y_{0}\right)\right)=0, f^{\prime}\left(y_{0}\right)=0, f^{\prime \prime}\left(y_{0}\right) \neq 0$, and $f^{\prime}(y) \neq 0$ in $\operatorname{supp}(u) \backslash\left\{y_{0}\right\}$. Here the phase function $f(t)=i\left(\phi(t)-\phi\left(T^{\prime}\right)\right)$ is imaginary (note that $\phi^{\prime \prime}\left(T^{\prime}\right)>0$ ), $\omega=2 \tau(s, \lambda, S)$, and $u=\phi^{k} \chi_{2}$. The stationary point is $t=T^{\prime}$. With $l_{1}=1$, we obtain

$$
\begin{array}{r}
\left|\int_{t_{0}}^{T} e^{-2 \tau(s, S)\left(\phi(t)-\phi\left(T^{\prime}\right)\right)} \phi^{k}(t) \chi_{2}(t) d t-\phi^{k}\left(T^{\prime}\right) \sqrt{\frac{\pi}{\phi^{\prime \prime}\left(T^{\prime}\right)}} \tau(s, \lambda, S)^{-\frac{1}{2}}\right| \\
\leq C \tau(s, \lambda, S)^{-3 / 2} .
\end{array}
$$

This yields (2.9).

To achieve our goal we also need an estimation from below for the terms $W_{i, j}(s, \lambda)$. We set

$$
\begin{aligned}
W_{k, j}^{(i)}(s, \lambda):=\int_{\Omega_{k, j}} e^{-2 s \eta^{(i)}\left(T^{\prime}, x\right)} \varphi^{(i)^{\frac{3}{2}}}\left(T^{\prime}, x\right) & \left|\Delta \widetilde{y}\left(T^{\prime}, x\right)\right|^{2} d x, \\
k & =0,1, j=1, \ldots, p_{k}, i=1,2 .
\end{aligned}
$$

For the terms $W_{0, j}^{(i)}(s, \lambda)$, we have the following

Lemma 2.6. Let $\varepsilon>0$. We have

$$
\begin{aligned}
& \qquad W_{0, j}^{(i)}(s, \lambda) \geq C_{s_{2}, i, j} \frac{r^{2}\left|S_{j}\right|}{s \lambda} e^{-2 s \eta^{(i)}\left(T^{\prime}, S\right)}\left(\varphi^{(i)}\left(T^{\prime}, S\right)\right)^{\frac{1}{2}} e^{-\lambda \varepsilon}, \\
& \qquad i=1,2, j=1, \ldots, p_{0},
\end{aligned}
$$


Proof. In the proof, we shall write $\beta$, etc, in place of $\beta^{(i)}$, etc. Taking $\delta$ sufficiently small, we start by choosing a small neighborhood $W$ of $S_{j}$ in $\bar{\Omega}_{0}$ globally parameterized by $(\sigma, y) \in[0, \delta] \times S_{j}$ (see the proof of Lemma A.5 in Appendix A). In fact, we can choose the coordinates and the small neighborhood of $S_{j}$ such that $\sigma=$ cst corresponds to level sets for the function $\beta$ (use $\nabla \beta$ for the vector field $v$ in proof of Lemma A.5). Note that in the neighborhood $W$ the function $\beta$ decreases with $\sigma$.

Estimating from below the Jacobian ${ }^{1}$ originating from the change of variable and observing that the integrand is constant w.r.t. $y$ we obtain

$$
\begin{aligned}
W_{0, j}(s, \lambda) & \geq C r^{2}\left|S_{j}\right| \int_{0}^{\delta} e^{-2 s \eta\left(T^{\prime}, \sigma\right)} \varphi^{\frac{3}{2}}\left(T^{\prime}, \sigma\right) d \sigma \\
& =C r^{2}\left|S_{j}\right| e^{-2 s \eta\left(T^{\prime}, S\right)} \int_{0}^{\delta} e^{-2 s\left(\eta\left(T^{\prime}, \sigma\right)-\eta\left(T^{\prime}, S\right)\right)} \varphi^{\frac{3}{2}}\left(T^{\prime}, \sigma\right) d \sigma .
\end{aligned}
$$

We now use the change of variables $\sigma^{\prime}=\eta\left(T^{\prime}, \sigma\right)-\eta\left(T^{\prime}, S\right) \geq 0$ which yields

$$
W_{0, j}(s, \lambda) \geq C r^{2}\left|S_{j}\right| \lambda^{-1} e^{-2 s \eta\left(T^{\prime}, S\right)} \int_{0}^{\delta^{\prime}} e^{-2 s \sigma^{\prime}} \varphi^{\frac{1}{2}}\left(T^{\prime}, \sigma\right)\left|\partial_{\sigma} \beta\right|^{-1} d \sigma^{\prime},
$$

where $\delta^{\prime}=\eta\left(T^{\prime}, \delta\right)-\eta\left(T^{\prime}, S\right)$. We can find in $W$ a positive lower bound for $\left(\partial_{\sigma} \beta\right)^{-1}$ independent of $\delta$, i.e. the size of $W$. We thus obtain

$$
\begin{aligned}
W_{0, j}(s, \lambda) & \geq C r^{2}\left|S_{j}\right| \lambda^{-1} e^{-2 s \eta\left(T^{\prime}, S\right)} \varphi^{\frac{1}{2}}\left(T^{\prime}, \delta\right) \int_{0}^{\delta^{\prime}} e^{-2 s \sigma^{\prime}} d \sigma^{\prime} \\
& \geq C r^{2}\left|S_{j}\right| s^{-1} \lambda^{-1} e^{-2 s \eta\left(T^{\prime}, S\right)} \varphi^{\frac{1}{2}}\left(T^{\prime}, \delta\right) \int_{0}^{s \delta^{\prime}} e^{-2 \sigma^{\prime}} d \sigma^{\prime} \\
& \geq C^{\prime}(s) r^{2}\left|S_{j}\right| s^{-1} \lambda^{-1} e^{-2 s \eta\left(T^{\prime}, S\right)} \varphi^{\frac{1}{2}}\left(T^{\prime}, \delta\right),
\end{aligned}
$$

with $C^{\prime}(s)$ increasing with $s$. Observe now that

$$
\begin{aligned}
\varphi\left(T^{\prime}, \delta\right)=\frac{e^{\lambda \beta(\delta)}}{\left(T-T^{\prime}\right)\left(T^{\prime}-t_{0}\right)}=\frac{e^{\lambda \beta(S)}}{\left(T-T^{\prime}\right)\left(T^{\prime}-t_{0}\right)} e^{\lambda(\beta(\delta)-\beta(S))} & =\varphi\left(T^{\prime}, S\right) e^{\lambda(\beta(\delta)-\beta(S))} .
\end{aligned}
$$

Choosing $\delta$ sufficiently small such that $\frac{1}{2}(\beta(S)-\beta(\delta)) \leq \varepsilon$ thus yields the result.

With the previous lemmas we can now prove that the coefficient of $\left|\xi_{0, j}\right|^{2}, j=$ $1, \ldots, p_{0}$, in $(2.8)$ can be made positive. This requires taking both $\lambda$ and $s$ sufficiently large.

Proposition 2.7. Let $1 \leq j \leq p_{0}$. There exists $\lambda_{2, j} \geq \lambda_{1}$ such that if $\lambda \geq \lambda_{2, j}$ then for s sufficiently large

$$
\begin{aligned}
& \qquad \begin{array}{c}
A_{0, j}=\left(1-C K^{\prime 2} s^{-\frac{3}{2}} \lambda^{-2}\right) W_{0, j}(s, \lambda)-C M^{\prime}\left[s^{-\frac{1}{2}} \lambda^{-1} w_{1}(s, \lambda)\right. \\
\left.\quad+s^{-\frac{3}{2}} \lambda^{-2} w_{4}(s, \lambda)+s^{-\frac{7}{2}} \lambda^{-2} w_{0}(s, \lambda)\right] \geq C(s, \lambda)>0,
\end{array} \\
& \text { with } C(s, \lambda)=C\left(s, \lambda, r, K, M, c_{\max }, c_{\min }, j\right)
\end{aligned}
$$

${ }^{1}$ Note that the estimation from below of the Jacobian is independent from the size of the neighborhood $W$. 
Proof. It suffices to prove the result for $w_{k}^{(i)}(s, \lambda)$ and $W_{0, j}^{(i)}(s, \lambda)$. We shall write $\beta$, etc, in place of $\beta^{(i)}$, etc. We take $s$ sufficiently large such that (1$\left.C K^{\prime 2} s^{-\frac{3}{2}} \lambda^{-2}\right) \geq c_{0}>0$.

From Lemmas 2.5 and 2.6 , for $\varepsilon>0$ we obtain

$$
\begin{aligned}
& A_{0, j} \geq-s^{-2} \nu(\lambda) C_{1} e^{-2 s \eta\left(T^{\prime}, S\right)} \\
&+\frac{1}{s \lambda} e^{-2 s \eta\left(T^{\prime}, S\right)}\left[\frac{C_{0}}{2} C_{\varepsilon} r^{2}\left|S_{j}\right|\left(\varphi\left(T^{\prime}, S\right)\right)^{\frac{1}{2}} e^{-\lambda \varepsilon}\right. \\
&\left.\quad-C M^{\prime} \frac{\sqrt{\pi} \varphi\left(T^{\prime}, S\right)}{\sqrt{\phi^{\prime \prime}\left(T^{\prime}\right)} \sqrt{e^{\lambda \bar{\beta}}-e^{\lambda \beta(S)}}}\right],
\end{aligned}
$$

where $\nu$ are a bounded function. We first treat the second term in the previous expression. Note that this term originates from the estimate from below for $W_{0, j}^{(i)}$ and the estimate of $s^{-\frac{1}{2}} \lambda^{-1} w_{1}^{(i)}$ by Lemma 2.5. The other terms in $A_{0, j}$ and the remainder part of the estimation of $s^{-\frac{1}{2}} \lambda^{-1} w_{1}^{(i)}$ are lumped in the first term of (2.10).

Choose now $\varepsilon<\frac{1}{2}(\bar{\beta}-\beta(S))$ (recall that $\bar{\beta}>\beta(S)$ because $m>1$ ). Then since $\bar{\beta}>\beta(S)$ we have

$$
\frac{\varphi\left(T^{\prime}, S\right)}{\sqrt{e^{\lambda \bar{\beta}}-e^{\lambda \beta(S)}}}=o\left(\left(\varphi\left(T^{\prime}, S\right)\right)^{\frac{1}{2}} e^{-\lambda \varepsilon}\right)
$$

for $\lambda$ large. Thus the second term can be made positive for $\lambda$, say $\lambda=\lambda_{2, j}$, sufficiently large.

Once $\lambda$ is fixed larger than $\lambda_{2, j}$, the first term in (2.10) can be made positive by taking $s$ sufficiently large.

We now prove that the coefficient of $\left|\xi_{1, j}\right|^{2}, j=1, \ldots, p_{1}$, in (2.8) can be made positive. Here, the parameter $\lambda$ is not of use.

Proposition 2.8. Let $1 \leq j \leq p_{1}$. Let $\lambda \geq \lambda_{1}$. Then for $s$ sufficiently large

$$
\begin{aligned}
A_{1, j}=\left(1-C K^{\prime 2} s^{-\frac{3}{2}} \lambda^{-2}\right) & W_{1, j}(s, \lambda)-C M^{\prime}\left[s^{-\frac{1}{2}} \lambda^{-1} w_{1}(s, \lambda)\right. \\
& \left.+s^{-\frac{3}{2}} \lambda^{-2} w_{4}(s, \lambda)+s^{-\frac{7}{2}} \lambda^{-2} w_{0}(s, \lambda)\right] \geq C(s, \lambda)>0,
\end{aligned}
$$

with $C(s, \lambda)=C\left(s, \lambda, r, K, M, c_{\max }, c_{\min }\right)$.

Proof. It suffices to prove the result for $w_{k}^{(i)}(s, \lambda)$ and $W_{1, j}^{(i)}(s, \lambda)$. We shall write $\beta$, etc, in place of $\beta^{(i)}$, etc. We take $s$ sufficiently large such that $(1-$ $\left.C K^{\prime 2} s^{-\frac{3}{2}} \lambda^{-2}\right) \geq C_{0}>0$.

We first write

$$
e^{-2 s \eta(t, S)}=e^{-2 s \eta\left(T^{\prime}, S\right)} e^{-2 s\left(\eta(t, S)-\eta\left(T^{\prime}, S\right)\right)},
$$


and observe that for $s \geq s_{0}>0$

$$
\int_{t_{0}}^{T} e^{-2 s\left(\eta(t, S)-\eta\left(T^{\prime}, S\right)\right)} \varphi^{k}(t, S) d t \leq L\left(s_{0}, \lambda, k\right),
$$

for some positive $L\left(s_{0}, \lambda, k\right)$. From Lemma 1.1, there exists $V \Subset \Omega_{1, j}$ such that $\inf _{x \in V} \beta>\beta(S)$. Then with

$$
\eta_{\max }^{T^{\prime}, V}=\sup _{x \in V} \frac{e^{2 \lambda K^{(i)}}-e^{\lambda \beta^{(i)}(x)}}{\left(T^{\prime}-t_{0}\right)\left(T-T^{\prime}\right)}
$$

we have $-\eta\left(T^{\prime}, x\right) \geq-\eta_{\max }^{T^{\prime}, V}>-\eta\left(T^{\prime}, S\right)$, for $x \in V$, and $s>0$. These observations yield

$$
W_{1, j}(s) \geq r^{2} \int_{V} e^{-2 s \eta\left(T^{\prime}, x\right)} \varphi^{\frac{3}{2}}\left(T^{\prime}, x\right) d x \geq C(\lambda) r^{2}|V| e^{-2 s \eta_{\max }^{T^{\prime}, V}},
$$

and

$$
w_{k}(s) \leq L\left(s_{0}, \lambda, k\right) e^{-2 s \eta\left(T^{\prime}, S\right)},
$$

which implies the result.

With (2.8) and Propositions 2.7 and 2.8, recalling that $v=u_{t}=\partial_{t}(y-\widetilde{y})$, We have thus obtained the following stability result.

Theorem 2.9. Let $\gamma$ be a subset of the boundary $\Gamma$ of an open set $\Omega$ of $\mathbb{R}^{n}$ that satisfies Condition (GC), and $\gamma$ satisfies Assumption 0.4. We assume that the diffusion coefficients $c$ and $\widetilde{c}$ satisfy Assumptions 0.2 and 2.4 and $c_{0}-c_{1} \geq$ $\Delta>0$. Let $y_{0}, \widetilde{y}_{0}$ in $L^{2}(\Omega)$ and let $y, \widetilde{y}$ be solutions to (2.1)-(2.2) satisfying Assumption 2.1. Then there exists a constant $C$

$$
C=C\left(\Omega, T, t_{0}, \gamma, S, \mathcal{O}^{(1)}, \mathcal{O}^{(2)}, M, K, r, c_{\min }, c_{\max }, \Delta\right)
$$

such that

$$
\sum_{i=0,1} \sum_{j=1}^{p_{i}}\left|c_{i j}-\widetilde{c}_{i j}\right|^{2} \leq C\left|\partial_{n}\left(\partial_{t} y-\partial_{t} \widetilde{y}\right)\right|_{L^{2}((0, T) \times \gamma)}+C\left|\Delta y\left(T^{\prime}, .\right)-\Delta \widetilde{y}\left(T^{\prime}, .\right)\right|_{L^{2}\left(\Omega^{\prime}\right)}^{2} .
$$

We shall see in Proposition 3.5, below, that we can achieve the regularity properties and estimates of Assumption 2.1.

Remark 2.10. Observe than in the statement of Theorem 2.9 the initial condition $y_{0}$ and $\widetilde{y}_{0}$ need not be equal (see systems $(2.1)-(2.2)$ ).

Remark 2.11. If the position of the interface $S$ is known, we can improve the result of Theorem 2.9 by locally observaing of the solutions $y$ and $\widetilde{y}$ at time $T^{\prime}$. Let $\omega=\omega_{0} \cup \omega_{1}$, with $\omega_{1}$ a neighborhood of $\gamma$ in $\bar{\Omega}_{1}$ and $\omega_{0}$ a neighborhood of $S$ in $\bar{\Omega}_{0}$. We can in fact relax Assumption 2.1 with the following one: Let $r>0$, 
the solution $\widetilde{y}$ is such that $\left|\Delta \widetilde{y}\left(T^{\prime}\right)\right| \geq r>0$ in $\omega$. Then, we obtain a stability estimate by solely observing $y$ and $\widetilde{y}$ at time $T^{\prime}$ on $\omega$ in place of $\Omega$

$$
\sum_{i=0,1} \sum_{j=1}^{p_{i}}\left|c_{i j}-\widetilde{c}_{i j}\right|^{2} \leq C\left|\partial_{n}\left(\partial_{t} y-\partial_{t} \widetilde{y}\right)\right|_{L^{2}((0, T) \times \gamma)}^{2}+C\left|\Delta y\left(T^{\prime}, .\right)-\Delta \widetilde{y}\left(T^{\prime}, .\right)\right|_{L^{2}(\omega)}^{2} .
$$

We briefly sketch the proof, as it closely follows the exposition of the proof of estimate (2.11) in this section. It relies on the following lemma.

Lemma 2.12. Let $s_{3}>0$ and $\lambda_{3}>0$. There exists $C>0$ such that for all $s \geq s_{3}$, and $\lambda \geq \lambda_{3}$, we have

$$
\begin{aligned}
\int_{\Omega_{i}}\left(e^{-2 s \eta^{(1)}} \varphi^{(1) \frac{3}{2}}\right. & \left.+e^{-2 s \eta^{(2)}\left(T^{\prime}, x\right)} \varphi^{(2)^{\frac{3}{2}}}\right)\left(T^{\prime}, x\right) d x \\
& \leq C \int_{\omega_{i}}\left(e^{-2 s \eta^{(1)}} \varphi^{(1)^{\frac{3}{2}}}+e^{-2 s \eta^{(2)}\left(T^{\prime}, x\right)} \varphi^{(2) \frac{3}{2}}\right)\left(T^{\prime}, x\right) d x
\end{aligned}
$$

Proof. Set $A=\sup _{\Omega_{1} \backslash \omega_{1}} \beta$ and let $\varepsilon>0$ be sufficiently small such that $B=$ $A+\varepsilon<\sup _{\Omega_{1}} \beta$ and define $\widetilde{\omega}_{1}=\left\{x \in \Omega_{1} ; A+\varepsilon<\beta(x)\right\}$. Then $\widetilde{\omega}_{1} \subset \omega_{1}$ and we set $K=\frac{\left|\Omega_{1} \backslash \omega_{1}\right|}{\left|\omega_{1}\right|}$. Defining $\eta_{B}^{(i)}$ and $\varphi_{B}^{(i)}$ to be equal to $\eta^{(i)}$ and $\varphi^{(i)}$ at time $T^{\prime}$ and $\beta(x)$ replaced by $B$. Then for all $s \geq s_{3}$, and $\lambda \geq \lambda_{3}$, we have

$$
\int_{\Omega_{1}} e^{-2 s \eta_{B}^{(i)}} \varphi_{B}^{(i) \frac{3}{2}} d x \leq K \int_{\omega_{1}} e^{-2 s \eta_{B}^{(i)}} \varphi_{B}^{(i) \frac{3}{2}} d x, \quad i=1,2 .
$$

We conclude by observing that, for $i=1,2$,

$$
\begin{aligned}
e^{-2 s \eta_{B}^{(i)}} & \leq e^{-2 s \eta^{(i)}\left(T^{\prime}, x\right)}, \quad \varphi_{B}^{(i)} \leq \varphi^{(i)}\left(T^{\prime}, x\right), \quad x \in \widetilde{\omega}_{1}, \\
e^{-2 s \eta^{(i)}\left(T^{\prime}, x\right)} & \leq e^{-2 s \eta_{B}^{(i)}}, \quad \varphi^{(i)}\left(T^{\prime}, x\right) \leq \varphi_{B}^{(i)}, \quad x \in \Omega_{1} \backslash \widetilde{\omega}_{1} .
\end{aligned}
$$

The same method can be applied for the second set of integrals on $\Omega_{0}$ and $\omega_{0}$.

Continuation of Remark 2.11. In the statement of Lemma 2.3 we can replace the integration domain, $\Omega^{\prime}$, in the l.h.s. of the estimate, by $\omega$.

To reach an equation of the form of (2.8) with the volume integrals computed ${ }^{2}$ over $\omega$ in place of $\Omega$ we write the following counterpart to (2.7):

$$
\begin{aligned}
& \sum_{i=0,1} \int_{t_{0}}^{T} \int_{\Omega_{i}}\left(e^{-2 s \eta^{(1)}}+e^{-2 s \eta^{(2)}}\right)\left|\xi \Delta \partial_{t} \widetilde{y}\right|^{2} d x d t \\
& \leq K^{\prime 2} \sum_{i=0,1} \sum_{j=1}^{p_{i}}\left|\xi_{i, j}\right|^{2} \int_{\Omega_{i, j} \cap \omega_{i}}\left(e^{-2 s \eta^{(1)}\left(T^{\prime}, x\right)}+e^{-2 s \eta^{(2)}\left(T^{\prime}, x\right)}\right)\left|\Delta \widetilde{y}\left(T^{\prime}, x\right)\right|^{2} d x
\end{aligned}
$$

by Lemma 2.12. The result of Lemma 2.6 remain unchanged as $\omega_{0}$ is a neighborhood of $S$ in $\bar{\Omega}_{0}$. In the proof of Proposition 2.8 we can choose the open set $V$ to be in $\omega_{1}$.

\footnotetext{
${ }^{2}$ including the definition of $W_{i, j}, i=0,1, j=1, \ldots, p_{i}$.
} 
Remark 2.13. Note that if we assume that $y\left(T^{\prime},.\right)=\widetilde{y}\left(T^{\prime},.\right)$ then the stability estimate becomes

$$
\sum_{i=0,1} \sum_{j=1}^{p_{i}}\left|c_{i j}-\widetilde{c}_{i j}\right|^{2} \leq C\left|\partial_{n}\left(\partial_{t} y-\partial_{t} \widetilde{y}\right)\right|_{L^{2}((0, T) \times \gamma)}^{2} .
$$

Such an additional assumption is sometimes made, e.g. in [19].

With Theorem 2.9 we have the following uniqueness result

Corollary 2.14. Under the same assumptions as Theorem 2.9 and if

$$
\begin{aligned}
& \partial_{n}\left(\partial_{t}(y-\widetilde{y})\right)(t, x)=0 \text { in }\left(t_{0}, T\right) \times \gamma, \\
& \Delta y\left(T^{\prime}, x\right)-\Delta \widetilde{y}\left(T^{\prime}, x\right)=0, \text { in } \Omega^{\prime},
\end{aligned}
$$

then $c=\widetilde{c}$. Furthermore $y_{0}=\widetilde{y}_{0}$.

Proof. The second assertion remains to be proved. If $c=\widetilde{c}$ then $u=y-\widetilde{y} \in \mathscr{D}_{A}$, with $A=\nabla \cdot(c \nabla()$.$) (see Appendix A), is solution to$

$$
\begin{cases}\partial_{t} u-\nabla \cdot(c \nabla u)=0 & \text { in }(0, T) \times \Omega, \\ u=0, & \text { on }(0, T) \times \Gamma, \\ u(0, x)=u_{0}(x), & \text { in } \Omega,\end{cases}
$$

with $u_{0}=y_{0}-\widetilde{y}_{0}$. Thus $u=S(t) u_{0}$. We have $\Delta(u)\left(T^{\prime}\right)=0$ in $\Omega^{\prime}$. Thus $\nabla \cdot(c \nabla u)_{\left.\right|_{\Omega^{\prime}}}\left(T^{\prime}\right)=0$. Since $u\left(T^{\prime}\right) \in \mathscr{D}_{A}$ we have $u\left(T^{\prime}\right)=0$. Since the semigroup $S(t)$ generated by $-\nabla \cdot(c \nabla()$.$) is analytic by Proposition A.3, we obtain that$ $S(t) u_{0}=0$ for all $t>0$. The continuity in $t=0^{+}$yields $u_{0}=0$.

If we make further assumptions on the initial conditions $y_{0}$ and $\widetilde{y}_{0}$ we can in fact obtain a stability result for these initial conditions as well. This is the subject of Section 4.

Remark 2.15. In the stability result obtained here, we have made the choice to make some of the measurements on part of the boundary $(0, T) \times \Gamma$. Derivation of a Carleman estimate, as in [7], with a r.h.s. with an 'observation' in an inner volume $(0, T) \times \omega$ of $(0, T) \times \Omega_{1}$ would yield a stability estimate like (2.11) with $\left|\partial_{t} y-\partial_{t} \widetilde{y}\right|_{L^{2}\left(\left(t_{0}, T\right) \times \omega\right)}$ in the r.h.s..

Remark 2.16. Observe that in place of Assumption 2.4, we could have solely assumed that the difference $\xi=c-\widetilde{c}$ is piecewise constant. Then, we would replace $c \Delta u$ by $\nabla \cdot(c \nabla u)$ in the r.h.s. of (2.8). This would yield a stability estimate of the form

$$
\begin{gathered}
\sum_{i=0,1} \sum_{j=1}^{p_{i}}\left|c_{i j}-\widetilde{c}_{i j}\right|^{2} \leq C\left|\partial_{n}\left(\partial_{t} y-\partial_{t} \widetilde{y}\right)\right|_{L^{2}((0, T) \times \gamma)}^{2}+C\left|\nabla y\left(T^{\prime}, .\right)-\nabla \widetilde{y}\left(T^{\prime}, .\right)\right|_{\left(L^{2}\left(\Omega^{\prime}\right)\right)^{n}}^{2} \\
+C\left|\Delta y\left(T^{\prime}, .\right)-\Delta \widetilde{y}\left(T^{\prime}, .\right)\right|_{L^{2}\left(\Omega^{\prime}\right)}^{2} .
\end{gathered}
$$

In Remark 3.7, in Section 3, we show that Assumption 2.1 can be fulfilled, in the case where $\xi$ is piecewise constant, if $|\nabla \widetilde{c}|$ is sufficiently small and $\widetilde{c}$ sufficiently regular. 
Remark 2.17. Here we have set the r.h.s. of the parabolic equations in (2.1) and (2.2) to zero. With a non-vanishing r.h.s., say $f(t, x)$, the same stability result holds once the hypothesis of Assumption 2.1 are fulfilled, since (2.3) is preserved. Assuming that the function $f(t, x)$ is bounded and sufficiently regular, say of class $\mathscr{C}^{k}$, with $k$ sufficiently large, we can obtain the same results as in Proposition 3.5 of Section 3, by a slight modification of the argumentation. In this case also, the class of solutions $y$ and $\widetilde{y}$ satisfying Assumption 2.1 is non empty.

\section{Existence of solutions $y, \widetilde{y}$ satisfying Assump- tion 2.1}

We propose a possible choice of boundary condition $h$ and of initial condition $\widetilde{y}_{0}$ to achieve the particular properties for the solutions $y$ and $\widetilde{y}$ listed in Assumption 2.1 needed in the proof of Theorem 2.9 in Section 2.

We shall denote $S(t)$ (resp. $\widetilde{S}(t))$ the analytic semi-group generated by unbounded operator $A$ (resp. $\widetilde{A}$ ) formally defined by $-\nabla \cdot(c \nabla()$.$) (resp. -\nabla$. $(\widetilde{c} \nabla())$.$) on L^{2}(\Omega)$ with domain (See appendix A)

$$
\begin{aligned}
& \mathscr{D}_{A}=\left\{u \in H_{0}^{1}(\Omega) ; \nabla \cdot(c \nabla u) \in L^{2}(\Omega)\right\}, \\
& \left(\operatorname{resp} . \mathscr{D}_{\widetilde{A}}=\left\{u \in H_{0}^{1}(\Omega) ; \nabla \cdot(\widetilde{c} \nabla u) \in L^{2}(\Omega)\right\}\right) .
\end{aligned}
$$

The convention we use here is ' $S(t)=e^{-t A}$ '.

Lemma 3.1. Let $r>0$ and let $\widetilde{c} \in L^{\infty}(\Omega)$. There exists $\widetilde{y}_{0} \in \mathscr{D}_{\widetilde{A}}$ and $\chi$ : $[0, T] \rightarrow \mathbb{R}$ such that the solution to

$$
\begin{cases}\partial_{t} \widetilde{y}-\nabla \cdot(\widetilde{c} \nabla \widetilde{y})=0, & \text { in }(0, T) \times \Omega, \\ \widetilde{y}(t, x)=\chi(t), & \text { on }(0, T) \times \Gamma, \\ \widetilde{y}(t, .)-\chi(t) \in \mathscr{D}_{\widetilde{A}}, & 0<t \leq T, \\ \widetilde{y}(0)=\widetilde{y}_{0}, & \end{cases}
$$

satisfies $\left|\nabla \cdot(\widetilde{c} \nabla \widetilde{y})\left(T^{\prime}\right)\right| \geq r>0$ a.e.. The function $\chi$ can be chosen such that $\chi^{\prime}$ is a positive constant.

Proof. Observe that $p(t, x)=\widetilde{y}(t, x)-\chi(t)$ is solution to

$$
\begin{cases}\partial_{t} p-\nabla \cdot(\widetilde{c} \nabla p)=-\chi^{\prime}(t), & \text { in }(0, T) \times \Omega, \\ p(t, x)=0, & \text { on }(0, T) \times \Gamma, \\ p(t, .) \in \mathscr{D}_{\widetilde{A}}, & 0<t \leq T, \\ p(0, x)=\widetilde{y}_{0}(x)-\chi(0)=p_{0} \in L^{2}(\Omega), & \end{cases}
$$

and is thus given by Duhamel's formula [21]

$$
p(t)=\widetilde{S}(t) p_{0}-\int_{0}^{t} \widetilde{S}(t-s) \chi^{\prime}(s) d s .
$$


In fact, we choose $\chi$ of the form $\chi(t)=-\rho t$, where $\rho$ is a negative constant. We also choose $\widetilde{y}_{0}$ such that $p_{0}=\widetilde{y}_{0} \in \mathscr{D}_{\widetilde{A}}$ and $\nabla \cdot\left(\widetilde{c} \nabla \widetilde{y}_{0}\right) \geq r_{0}>r$ a.e. in $\Omega$ (choose $f \in L^{2}(\Omega)$, such that $f>r_{0}$, and solve the elliptic problem $\nabla \cdot\left(\widetilde{c} \nabla \widetilde{y}_{0}\right)=f$ for $\widetilde{y}_{0}$ in $\left.H_{0}^{1}(\Omega)\right)$. We choose $\rho$ such that $-r_{0}<\rho \leq-r<0$.

The solution $p$ to $(3.2)$ is unique in $\mathscr{C}^{1}\left([0, T], L^{2}(\Omega)\right) \cap \mathscr{C}^{0}\left([0, T], \mathscr{D}_{\widetilde{A}}\right)$ and given by (3.3) [6, Theorem 3 and following Remark 2, Section XVII B.1]. Denoting by 1 the function identically equal to 1 on $\Omega$ we find

$$
p(t)=\widetilde{S}(t) p_{0}+\rho \int_{0}^{t} \widetilde{S}(s) \mathbf{1} d s,
$$

which yields $q:=-\widetilde{A} p+\rho \mathbf{1}:=\widetilde{S}(t)\left(\nabla \cdot\left(\widetilde{c} \nabla p_{0}\right)+\rho \mathbf{1}\right)[21$, Theorem 1.2.4]. Hence $q$ is the solution to

$$
\begin{cases}\partial_{t} q-\nabla \cdot(\widetilde{c} \nabla q)=0, & \text { in }(0, T) \times \Omega, \\ q(t, x)=0, & \text { on }(0, T) \times \Gamma \\ q(t, .) \in \mathscr{D}_{\widetilde{A}}, & 0<t \leq T \\ q(0, x)=q_{0}(x):=\nabla \cdot\left(\widetilde{c} \nabla p_{0}\right)+\rho \mathbf{1} . & \end{cases}
$$

We now apply the maximum principle (which is valid for $L^{\infty}$ diffusion coefficients) [5, proof of Theorem IX.3] which reads for the time interval $\left[0, T^{\prime}\right]$

$$
\text { ess } \inf _{\overline{\mathrm{Q}}_{\mathrm{T}^{\prime}}} \mathrm{q} \geq \min \left(0, \operatorname{ess} \inf _{\Omega} \mathrm{q}_{0}\right)=0, \quad \mathrm{Q}_{\mathrm{T}^{\prime}}=\left(0, \mathrm{~T}^{\prime}\right) \times \Omega \text {. }
$$

This yields $\nabla \cdot(\widetilde{c} \nabla p)\left(T^{\prime}, x\right) \geq-\rho \geq r>0$ a.e..

Lemma 3.2. Let $l>n / 2$ and $l \geq 2$. Let $\widetilde{c} \in L^{\infty}(\Omega)$ be such that $\widetilde{c}_{\Omega_{i}}$ is $\mathscr{C}^{l-1}\left(\bar{\Omega}_{i}\right), i=0,1$. Let $\Omega$ be such that $S$ and $\partial \Omega$ are of class $\mathscr{C}^{l}$. Let $\widetilde{y}_{0} \in$ $\mathscr{D}_{\widetilde{A}}$ and the function $\chi:[0 . T] \rightarrow \mathbb{R}$, such that $\chi^{\prime}$ is constant, be both chosen according to Lemma 3.1. Then $\nabla \cdot(\widetilde{c} \nabla \widetilde{y})_{\left.\right|_{\Omega_{i}}} \in \mathscr{C}^{k}\left((0, T], L^{\infty}\left(\Omega_{i}\right)\right), i=0,1$, for all $k \in \mathbb{N}$. Let $\varepsilon>0$ then $\nabla \cdot(\widetilde{c} \nabla \widetilde{y})_{\left.\right|_{\Omega_{i}}}$, $i=0,1$, remain in a bounded domain of $\mathscr{C}^{k}\left([\varepsilon, T], L^{\infty}\left(\Omega_{i}\right)\right)$ for all $k \in \mathbb{N}$, uniformly w.r.t. $\widetilde{c}$ and $\widetilde{y}_{0}$, for $0<c_{\min } \leq \widetilde{c} \leq c_{\max }$ and $\nabla \cdot\left(\widetilde{c} \nabla \widetilde{y}_{0}\right)$ in a bounded domain of $L^{2}(\Omega)$.

Proof. We use the notations of the proof of Lemma 3.1. We set $p(t, x)=$ $\widetilde{y}(t, x)-\chi(t)$ and observe that $q:=-\widetilde{A} p+\rho \mathbf{1}$ is the solution to

$$
\begin{cases}\partial_{t} q-\nabla \cdot(\widetilde{c} \nabla q)=0, & \text { in }(0, T) \times \Omega, \\ q(t, x)=0, & \text { on }(0, T) \times \Gamma, \\ q(t, .) \in \mathscr{D}_{\widetilde{A}}, & 0<t \leq T, \\ q(0, x)=q_{0}(x):=\nabla \cdot\left(\widetilde{c} \nabla p_{0}\right)+\rho \mathbf{1} . & \end{cases}
$$

From Corollary A.11 we have that $q_{\left.\right|_{(0, T] \times \Omega_{i}}} \in \mathscr{C}^{k}\left((0, T] ; H^{l}\left(\Omega_{i}\right)\right), i=0,1$, for all $k \in \mathbb{N}$. Since $l>n / 2$, the space $H^{l}\left(\Omega_{i}\right)$ is continuously embedded in $L^{\infty}\left(\Omega_{i}\right)$ which yields the result. The last statement follows from Remark A.12.

Remark 3.3. In the case of $n=2,3$, which concerns most of the applications, we choose $m=2$. The condition on $S, \partial \Omega$ and the coefficients $\widetilde{c}_{\left.\right|_{\Omega_{i}}}$ in the previous lemma are then the default ones assumed in the introduction. 
Let the function $\chi$, such that $\rho=-\chi^{\prime}$ is constant, be chosen according to Lemma 3.1. We then have the following regularity property.

Lemma 3.4. Let $c, \widetilde{c} \in L^{\infty}(\Omega)$ be such that $c_{\left.\right|_{\Omega_{i}}}, \widetilde{c}_{\left.\right|_{\Omega_{i}}}$ is $\mathscr{C}^{1}\left(\bar{\Omega}_{i}\right), i=0,1,0<$ $c_{\min } \leq c, \widetilde{c} \leq c_{\max }$ and $y_{0}, \widetilde{y}_{0} \in L^{2}(\Omega)$ remain in a bounded domain of $L^{2}(\Omega)$. The solutions $\widetilde{y}$ and $y$ to

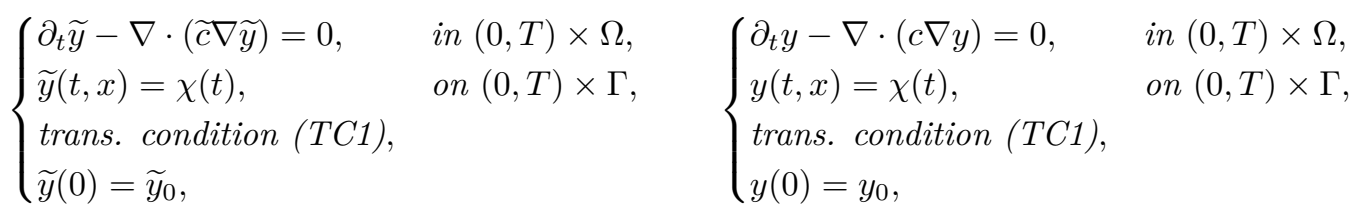

belong to $\mathscr{C}^{k}\left((0, T], H^{1}(\Omega)\right)$ and are such that $\widetilde{y}_{\mid \Omega_{i}}, y_{\left.\right|_{\Omega_{i}}} \in \mathscr{C}^{k}\left((0, T], H^{2}\left(\Omega_{i}\right)\right)$, $i=0,1$, for all $k \in \mathbb{N}$. Let $\varepsilon>0$, then for all $k \in \mathbb{N}, \widetilde{y}_{\left.\right|_{\Omega_{i}}}, y_{\left.\right|_{\Omega_{i}}}$ remain in a bounded domain of $\mathscr{C}^{k}\left((\varepsilon, T], H^{2}\left(\Omega_{i}\right)\right), i=0,1$, uniformly w.r.t. $\widetilde{c}, y_{0}$, and $\widetilde{y}_{0}$.

Proof. We work out the proof for $y$. We define $p(t, x)=y(t, x)-\chi(t)$. The function $p$ is solution to

$$
\begin{cases}\partial_{t} p-\nabla \cdot(c \nabla p)=\rho, & \text { in }(0, T) \times \Omega, \\ p(t, x)=0, & \text { on }(0, T) \times \Gamma, \\ p(t, .) \in \mathscr{D}_{A}, & 0<t \leq T, \\ p(0)=p_{0}=y_{0}-\chi(0) \in L^{2}(\Omega) . & \end{cases}
$$

It suffices to prove the result for $p$. Since $\rho$ is constant, the (mild) solution to (3.4) is a classical solution [21, Theorem 4.3.2]. We prove below that $p \in \mathscr{C}^{k}\left((0, T], \mathscr{D}_{A}\right), k>0$. Thus $p \in \mathscr{C}^{k}\left((0, T], L^{2}(\Omega)\right)$. Since $D_{A} \subset H_{0}^{1}$ with continuous injection then $p \in \mathscr{C}^{k}\left((0, T], H_{0}^{1}(\Omega)\right)$. By Proposition A.4 the maps $p \mapsto p_{\left.\right|_{\Omega_{i}}}, i=0,1$, are continuous from $\mathscr{D}_{A}$ into $H^{2}\left(\Omega_{i}\right)$. Thus $p_{\mid \Omega_{i}} \in \mathscr{C}^{k}\left((0, T], H^{2}\left(\Omega_{i}\right)\right)$.

The solution $p$ is given by

$$
p(t)=S(t) p_{0}+\rho \int_{0}^{t} S(s) \mathbf{1} d s,
$$

where 1 is the function identically equal to 1 on $\Omega$. The first term $p_{1}=S(t) p_{0}$ in $\mathscr{C}^{k}\left((0, T], \mathscr{D}_{A^{l}}\right)$ for all $k, l>0$ by Proposition A.2. For the second term $p_{2}=\rho \int_{0}^{t} S(s) \mathbf{1} d s$ we have [21, Theorem 1.2.4] $-A p_{2}=\rho(S(t) \mathbf{1}-\mathbf{1})$. Thus $A p_{2} \in$ $\mathscr{C}^{k}\left((0, T], L^{2}(\Omega)\right)$, i.e. $p_{2} \in \mathscr{C}^{k}\left((0, T], \mathscr{D}_{A}\right)$, for all $k>0$. The boundedness statement follows from Remark A.12.

With the proposed initial condition $\widetilde{y}_{0}$ and boundary condition $h(t, x)=\chi(t)$ we have thus obtained the following regularity and boundedness properties.

Proposition 3.5. Let $r>0$. Let $y_{0} \in L^{2}(\Omega)$. Let $\Omega$ be such that $S$ and $\Gamma=\partial \Omega$ are of class $\mathscr{C}^{l}$ and $\widetilde{c} \in L^{\infty}(\Omega)$ be such that $\widetilde{c}_{\left.\right|_{\Omega_{i}}}$ is $\mathscr{C}^{l-1}\left(\bar{\Omega}_{i}\right), i=0,1$, with $>n / 2, l \geq 2$. There exists $h(t, x) \in \mathscr{C}([0, T] \times \Gamma)$ and an initial condition $\widetilde{y}_{0} \in \mathscr{D}_{\widetilde{A}}$ such that the solutions $y, \widetilde{y}$ to systems (2.1)-(2.2) satisfy 
1. $\nabla \cdot(\widetilde{c} \nabla \widetilde{y})\left(T^{\prime}\right) \geq r>0$;

2. $\nabla \cdot(\widetilde{c} \nabla \widetilde{y})_{\left.\right|_{\Omega_{i}}} \in \mathscr{C}^{k}\left(\left[t_{0}, T\right], L^{\infty}\left(\Omega_{i}\right)\right), i=0,1$, for all $k \in \mathbb{N}$;

3. $y, \widetilde{y} \in \mathscr{C}^{k}\left(\left[t_{0}, T\right], L^{2}(\Omega)\right) \cap \mathscr{C}^{k}\left(\left(t_{0}, T\right], H^{1}(\Omega)\right)$, for all $k \in \mathbb{N}$;

4. $y_{\left.\right|_{\Omega_{i}}}, \widetilde{y}_{\Omega_{\Omega_{i}}} \in \mathscr{C}^{k}\left(\left[t_{0}, T\right], H^{2}\left(\Omega_{i}\right)\right), i=0,1$, for all $k \in \mathbb{N}$.

The restrictions $\widetilde{y}_{\Omega_{i}}$ remain in a bounded domain of $\mathscr{C}^{k}\left(\left[t_{0}, T\right], H^{2}\left(\Omega_{i}\right)\right)$ and $\nabla \cdot(\widetilde{c} \nabla \widetilde{y})_{\left.\right|_{\Omega_{i}}}$ in a bounded domain of $\mathscr{C}^{k}\left(\left[t_{0}, T\right], L^{\infty}\left(\Omega_{i}\right)\right)$, uniformly w.r.t. $\widetilde{c}$ and $\widetilde{y}_{0}$ if $0<c_{\min } \leq \widetilde{c} \leq c_{\max }$ and $\nabla \cdot\left(\widetilde{c} \nabla \widetilde{y}_{0}\right)$ remain in a bounded domain of $L^{2}(\Omega)$.

With Proposition 3.5 we observe that Assumption 2.1 in Section 2 can be fulfilled in the framework of Assumption 2.4 when $c_{\min } \leq \widetilde{c} \leq c_{\max }$ and $\widetilde{y}_{0} \in \mathscr{D}_{\widetilde{A}}$ such that $\nabla \cdot\left(\widetilde{c} \nabla \widetilde{y}_{0}\right)$ remain in a bounded domain of $L^{2}(\Omega)$ for properly chosen boundary conditions $h(t, x)$.

Remark 3.6. Observe that we could simply assume that $\widetilde{y}_{0} \in L^{2}(\Omega)$ and design the boundary condition $h(t, x)$ to reach a proper state in $\mathscr{D}_{\widetilde{A}}$ in a finite time $t_{1}<t_{0}$. This can be achieved as the parabolic equation we study here is null-controllable, i.e. exactly controllable to the trajectories [7].

Remark 3.7. In the case where we only assume that $\xi$ is piecewise constant, then from Item 1 of Proposition 3.5, we can obtain Item 1 of Assumption 2.1 , in the case where $|\nabla \widetilde{c}|$ is sufficiently small, since, from the proof of Lemma 3.2, we also find that $\nabla \widetilde{y}_{\left.\right|_{\Omega_{i}}}, i=0,1$, remain in bounded domains of $\mathscr{C}^{k}\left([\varepsilon, T],\left(L^{\infty}\left(\Omega_{i}\right)\right)^{n}\right)$. Note that we need to assume a sufficiently large regularity on the coefficient $\widetilde{c}$ in the proof of Lemma 3.2.

\section{Uniqueness and stability estimate for the ini- tial conditions}

In this section we closely follow the method of [22]. We shall assume

Assumption 4.1. Let $r_{0}>0$. The initial conditions $y_{0}$ and $\widetilde{y}_{0}$ satisfy

1. $y_{0}$ is in a bounded domain of $\mathscr{D}_{A}$;

2. $\widetilde{y}_{0}$ is in a bounded domain of $\mathscr{D}_{\widetilde{A}}$;

3. $\nabla \cdot\left(\widetilde{c} \nabla \widetilde{y}_{0}\right) \geq r_{0}$,

4. $y, \widetilde{y}$ are in a bounded domain of $\mathscr{C}^{1}\left([0, T], L^{2}(\Omega)\right)$, where $y$ and $\widetilde{y}$ are the solutions to (2.1)-(2.2).

Observe that item 4 of Assumption 4.1 implies that $\widetilde{y}_{\left.\right|_{\Omega_{i}}}, y_{\left.\right|_{\Omega_{i}}}$ are in a bounded domain of $\mathscr{C}\left([0, T], H^{2}\left(\Omega_{i}\right)\right), i=0,1$ by Proposition A.4. 
We denote $\widetilde{z}=\partial_{t} \widetilde{y} \in \mathscr{C}\left([0, T], L^{2}(\Omega)\right)$ and thus $\widetilde{z}(0)$ is well defined in $L^{2}(\Omega)$. We introduce $w$ the solution to

$$
\begin{cases}\partial_{t} w-\nabla \cdot(c \nabla w)=0, & \text { in }(0, T) \times \Omega, \\ w(t, x)=\partial_{t} h(t, x), & \text { on }(0, T) \times \Gamma, \\ \text { transmission conditions }(\mathrm{TC} 1), & \text { on }(0, T) \times S, \\ w(0)=\widetilde{z}(0), & \end{cases}
$$

and we further assume

Assumption 4.2. The functions $\widetilde{z}, w$ are in a bounded domain of $L^{2}\left(0, T, H^{1}(\Omega)\right)$.

We also assume that the diffusion coefficients $c$ and $\widetilde{c}$ are piecewise constant (Assumption 2.4).

Observe that if we choose the boundary condition $h(t, x)=-\rho t$ for $0<t \leq T$ according to the proof of Lemma 3.1 (with $0<r<r_{0}$ ), then the above assumption are fullfiled. In fact, the results of Section 3 show that Assumption 2.1 is then satisfied. In addition, item 4 in Assumption 4.1 and Assumption 4.2 are fulfilled by the following lemma.

Lemma 4.3. If $h(t, x)=-\rho$ then the solutions $y, \widetilde{y}$ to (2.1)-(2.2) and $w$ to (4.1) satisfy item 4 in Assumption 4.1 and Assumption 4.2.

Proof. We prove $w \in L^{2}\left(0, T, H^{1}(\Omega)\right)$. The proof is the same for $\widetilde{z}$. Let $p(t, x)=$ $w(t, x)-\partial_{t} h(t, x)=w(t, x)+\rho$. Then $p$ satisfies

$$
\begin{cases}\partial_{t} p-\nabla \cdot(c \nabla p)=0, & \text { in }(0, T) \times \Omega, \\ p(t, x)=0, & \text { on }(0, T) \times \Gamma, \\ \text { transmission conditions }(\mathrm{TC} 1), & \text { on }(0, T) \times S, \\ p(0)=\widetilde{z}(0)+\rho \in L^{2}(\Omega), & \end{cases}
$$

We thus have the usual energy estimate

$$
\frac{1}{2}|p(t)|_{L^{2}(\Omega)}^{2}+\int_{0}^{t} \int_{\Omega} c|\nabla p|^{2} d t d x=\frac{1}{2}|p(0)|_{L^{2}(\Omega)}^{2},
$$

and $p$, and thus $w$, is in $L^{2}\left(0, T, H^{1}(\Omega)\right)$ and remains in a bounded domain of this space if $c_{\min } \leq c \leq c_{\max }$ and $\widetilde{y}_{0}$ remains in a bounded domain of $\mathscr{D}_{\widetilde{A}}$.

To prove that $y$ is in a bounded domain of $\mathscr{C}^{1}\left([0, T], L^{2}(\Omega)\right.$ ) (the proof is the same for $\widetilde{y}$ ), we set $p(t, x)=y(t, x)+\rho t$ and observe that $q:=-A p+\rho \mathbf{1}$ is $\mathscr{C}\left([0, T], L^{2}(\Omega)\right)$ and thus $p \in \mathscr{C}\left([0, T], \mathscr{D}_{A}\right)$. Then $p \in \mathscr{C}^{1}\left([0, T], L^{2}(\Omega)\right)$.

Define $v_{1}$ and $v_{2}$ that satisfy

$$
\begin{cases}\partial_{t} v_{1}-\nabla \cdot\left(c \nabla v_{1}\right)=\nabla \cdot\left(\xi \nabla \partial_{t} \widetilde{y}\right), & \text { in }(0, T) \times \Omega^{\prime}, \\ v_{1}=0, & \text { on }(0, T) \times \Gamma, \\ \text { trans. conditions }(\mathrm{TC} 2), & \text { on }(0, T) \times S, \\ v_{1}(0)=0, & \end{cases}
$$


and

$$
\begin{cases}\partial_{t} v_{2}-\nabla \cdot\left(c \nabla v_{2}\right)=0, & \text { in }(0, T) \times \Omega^{\prime}, \\ v_{2}=0, & \text { on }(0, T) \times \Gamma, \\ \text { trans. conditions }(\mathrm{TC} 1), & \text { on }(0, T) \times S, \\ v_{2}(0)=\partial_{t}(y-\widetilde{y})(0) . & \end{cases}
$$

Observe that $\partial_{t}(y-\widetilde{y})(0)$ is well defined and in a bounded domain of $L^{2}(\Omega)$ by Assupmtion 4.1.

With an argument of logarithmic convexity we have

$$
\left|v_{2}(t)\right|_{L^{2}(\Omega)} \leq K^{1-t / T^{\prime}}\left|v_{2}\left(T^{\prime}\right)\right|_{L^{2}(\Omega)}^{t / T^{\prime}}, \quad 0 \leq t \leq T^{\prime} .
$$

Such an estimate makes use of the convexity of $F(t)=\ln \left(\left|v_{2}(t)\right|_{L^{2}(\Omega)}^{2}\right)$ and $\left|v_{2}(0)\right|_{L^{2}(\Omega)} \leq K$ (see [20, Section 2.3] for further details).

We now prove the following lemma

Lemma 4.4. There exists $C>0$, such that

$$
\left|v_{1}(t)\right|_{L^{2}(\Omega)} \leq C|c-\widetilde{c}|_{L^{\infty}(\Omega)}^{\frac{1}{2}}, \quad 0 \leq t \leq T .
$$

Note that $v_{1}$ satisfies transmission condition (TC2) and thus does not belong to $\mathscr{D}_{A}$. We thus cannot use some argument of regularity w.r.t. the source term from parabolic theory for $v_{1}$.

Proof. First observe that $v_{1}=w-\widetilde{z}$. From Assumption 4.2, $w$ and $\widetilde{z}$ remain in a bounded domain of $L^{2}\left(0, T, H^{1}(\Omega)\right)$. Now $\partial_{t}(w-\widetilde{z})-\nabla \cdot(c \nabla w-\widetilde{c} \nabla \widetilde{z})=0$, which after multiplication by $w-\widetilde{z}$, integration over $\Omega$ and an integration by parts, yields

$$
\begin{aligned}
& 0=\frac{1}{2} \partial_{t} \int_{\Omega}|w-\widetilde{z}|^{2} d x+\int_{\Omega}(c \nabla w-\widetilde{c} \nabla \widetilde{z}) \cdot(\nabla w-\nabla \widetilde{z}) d x \\
& =\frac{1}{2} \partial_{t} \int_{\Omega}|w-\widetilde{z}|^{2} d x+\int_{\Omega}(c-\widetilde{c}) \nabla w \cdot(\nabla w-\nabla \widetilde{z}) d x+\int_{\Omega} \widetilde{c}|\nabla w-\nabla \widetilde{z}|^{2} d x
\end{aligned}
$$

since $\nabla \cdot(c \nabla w-\widetilde{c} \nabla \widetilde{z}) \in L^{2}(\Omega)$ by the definitions of $\mathscr{D}_{A}$ and $\mathscr{D}_{\widetilde{A}}$ (see Appendix A). We thus obtain

$$
\begin{aligned}
\frac{1}{2} \partial_{t} \int_{\Omega}|w-\widetilde{z}|^{2} d x \leq \mid \int_{\Omega}(c-\widetilde{c}) & \nabla w \cdot(\nabla w-\nabla \widetilde{z}) d x \mid \\
& \leq|c-\widetilde{c}|_{L^{\infty}(\Omega)}|\nabla w|_{L^{2}(\Omega)}|\nabla w-\nabla \widetilde{z}|_{L^{2}(\Omega)}
\end{aligned}
$$

Integrating over $(0, t)$ yields the result.

As in Section 2, we define $v=\partial_{t}(y-\widetilde{y})$ and observe that $v=v_{1}+v_{2}$. We thus have

$$
\begin{array}{r}
|v(t)|_{L^{2}(\Omega)} \leq\left|v_{1}(t)\right|_{L^{2}(\Omega)}+\left|v_{2}(t)\right|_{L^{2}(\Omega)} \leq C\left(|c-\widetilde{c}|_{L^{\infty}(\Omega)}^{\frac{1}{2}}+\left|v_{2}\left(T^{\prime}\right)\right|_{L^{2}(\Omega)}^{t / T^{\prime}}\right) \\
0 \leq t \leq T^{\prime},
\end{array}
$$


and

$$
\begin{aligned}
\left|v_{2}\left(T^{\prime}\right)\right|_{L^{2}(\Omega)} \leq & \left|v\left(T^{\prime}\right)\right|_{L^{2}(\Omega)}+\left|v_{1}\left(T^{\prime}\right)\right|_{L^{2}(\Omega)} \\
& \leq C\left(\left|(\Delta y-\Delta \widetilde{y})\left(T^{\prime}\right)\right|_{L^{2}(\Omega)}+|c-\widetilde{c}|_{L^{\infty}(\Omega)}+|c-\widetilde{c}|_{L^{\infty}(\Omega)}^{\frac{1}{2}}\right),
\end{aligned}
$$

making use of

$$
v=\nabla \cdot(c \nabla u)+\nabla(\xi \cdot \nabla \widetilde{y})=c(\Delta y-\Delta \widetilde{y})+\xi \Delta \widetilde{y}, \quad \text { in } \Omega^{\prime},
$$

where Assumption 2.4 was applied. This yields

$$
|v(t)|_{L^{2}(\Omega)} \leq C\left(|c-\widetilde{c}|_{L^{\infty}(\Omega)}^{\frac{1}{2}}+\nu^{t / T^{\prime}}\right), \quad 0 \leq t \leq T^{\prime},
$$

with $\nu=\left|(y-\widetilde{y})\left(T^{\prime}\right)\right|_{H^{2}\left(\Omega^{\prime}\right)}+|c-\widetilde{c}|_{L^{\infty}(\Omega)}+|c-\widetilde{c}|_{L^{\infty}(\Omega)}^{\frac{1}{2}}$. Because of the regularity of $v$ w.r.t. time $t$ we now have

$$
\begin{aligned}
\left|y_{0}-\widetilde{y}_{0}\right|_{L^{2}(\Omega)}=\left|u_{0}\right|_{L^{2}(\Omega)}=\left|\int_{0}^{T^{\prime}} v(t) d t-u\left(T^{\prime}\right)\right|_{L^{2}(\Omega)} \\
\quad \leq \int_{0}^{T^{\prime}}|v(t)|_{L^{2}(\Omega)} d t+\left|y\left(T^{\prime}\right)-\widetilde{y}\left(T^{\prime}\right)\right|_{L^{2}(\Omega)},
\end{aligned}
$$

which gives

$$
\left|y_{0}-\widetilde{y}_{0}\right|_{L^{2}(\Omega)} \leq C\left(T^{\prime} \frac{\nu-1}{\ln (\nu)}+T^{\prime}|c-\widetilde{c}|_{L^{\infty}(\Omega)}^{\frac{1}{2}}\right)+\left|y\left(T^{\prime}\right)-\widetilde{y}\left(T^{\prime}\right)\right|_{L^{2}(\Omega)} .
$$

We thus have

$$
\left|y_{0}-\widetilde{y}_{0}\right|_{L^{2}(\Omega)} \leq C\left(\frac{\nu-1}{\ln (\nu)}+C^{\prime} \nu\right) .
$$

Observing that $x<\frac{x-1}{\ln (x)}$ for $x \in(0,1)$ we obtain

$$
\left|y_{0}-\widetilde{y}_{0}\right|_{L^{2}(\Omega)} \leq C \frac{\nu-1}{\ln (\nu)}, \quad \text { if } \nu<1 .
$$

With Theorem 2.9 we obtain

$$
|c-\widetilde{c}|_{L^{\infty}(\Omega)}^{2} \leq C\left|\partial_{n}\left(\partial_{t} y-\partial_{t} \widetilde{y}\right)\right|_{L^{2}((0, T) \times \gamma)}^{2}+C\left|\Delta y\left(T^{\prime}, .\right)-\Delta \widetilde{y}\left(T^{\prime}, .\right)\right|_{L^{2}\left(\Omega^{\prime}\right)}^{2} .
$$

When the r.h.s. of (4.5) is sufficiently small, we obtain

$$
0<\nu \leq C\left(\left|\partial_{n}\left(\partial_{t} y-\partial_{t} \widetilde{y}\right)\right|_{L^{2}((0, T) \times \gamma)}^{2}+\left|\Delta y\left(T^{\prime}, .\right)-\Delta \widetilde{y}\left(T^{\prime}, .\right)\right|_{L^{2}\left(\Omega^{\prime}\right)}^{2}\right)^{\frac{1}{4}}<1 .
$$

In that case

$$
\ln (\nu) \leq C^{\prime} \ln \left(C\left|(y-\widetilde{y})\left(T^{\prime}\right)\right|_{H^{2}\left(\Omega^{\prime}\right)}^{2}+C\left|\partial_{n}\left(\partial_{t} y-\partial_{t} \widetilde{y}\right)\right|_{L^{2}((0, T) \times \gamma)}^{2}\right)<0,
$$

and thus

$$
\frac{\nu-1}{\ln (\nu)} \leq C^{\prime}(\nu-1) / \ln \left(C\left|(y-\widetilde{y})\left(T^{\prime}\right)\right|_{H^{2}\left(\Omega^{\prime}\right)}^{2}+C\left|\partial_{n}\left(\partial_{t} y-\partial_{t} \widetilde{y}\right)\right|_{L^{2}((0, T) \times \gamma)}^{2}\right) .
$$

We thus obtain the following stability theorem for the initial conditions. 
Theorem 4.5. Under the hypothesis of Theorem 2.9 in addition to Assumptions 4.1 and 4.2 there exist some constants $C, C^{\prime}>0$

$$
\begin{aligned}
C & =C\left(\Omega, T, t_{0}, \gamma, S, \mathcal{O}^{(1)}, \mathcal{O}^{(2)}, M, K, r, c_{\min }, c_{\max }, \Delta\right), \\
C^{\prime} & =C^{\prime}\left(\Omega, T, t_{0}, \gamma, S, \mathcal{O}^{(1)}, \mathcal{O}^{(2)}, M, K, r, c_{\min }, c_{\max }, \Delta\right)
\end{aligned}
$$

such that

$$
\left|y_{0}-\widetilde{y}_{0}\right|_{L^{2}(\Omega)} \leq C^{\prime} /\left|\ln \left(C\left|(y-\widetilde{y})\left(T^{\prime}\right)\right|_{H^{2}\left(\Omega^{\prime}\right)}+C\left|\partial_{n}\left(\partial_{t} y-\partial_{t} \widetilde{y}\right)\right|_{L^{2}((0, T) \times \gamma)}\right)\right|
$$

for $\left|(y-\widetilde{y})\left(T^{\prime}\right)\right|_{H^{2}\left(\Omega^{\prime}\right)}+\left|\partial_{n}\left(\partial_{t} y-\partial_{t} \widetilde{y}\right)\right|_{L^{2}((0, T) \times \gamma)}$ sufficiently small.

\section{A generalization to embedded materials}

Let $\Omega \subset \mathbb{R}^{n}$ be a bounded connected open set. As before $\bar{\Omega}$ is assumed to be a $\mathscr{C}^{2}$ submanifold with boundary in $\mathbb{R}^{n}$. We consider some open sets $V_{0}, \ldots, V_{m}$ such that

$$
V_{0} \Subset V_{1} \Subset \cdots \Subset V_{m}=\Omega .
$$

We then set $\Omega_{i}:=V_{i} \backslash \bar{V}_{i-1}, i=1, \ldots, m$ and $\Omega_{0}=V_{0}$. Each open set $\Omega_{i}$, $i=0, \ldots, m$, is assumed to have $p_{i}$ connected components, $\Omega_{i, j}, j=1, \ldots, p_{i}$. We let $S_{i-1}^{i}=\bar{\Omega}_{i-1} \cap \bar{\Omega}_{i}$ be the interface between $\Omega_{i-1}$ and $\Omega_{i}, i=1, \ldots, m$. The interface $S_{i-1}^{i}$ is assumed to be $\mathscr{C}^{2}$. Similarly, we denote by $S_{i-1, j}^{i, k}$ the interface (possibly empty) between $\Omega_{i-1, j}$ and $\Omega_{i, k}$. We also set

$$
\begin{aligned}
& S_{i, j}=\bigcup_{k=1, \ldots, p_{i+1}} S_{i, j}^{i+1, k}, \text { for } i=0, \ldots, m-1, \\
& S^{i, j}=\bigcup_{k=1, \ldots, p_{i-1}} S_{i-1, k}^{i, j}, \text { for } i=1, \ldots, m,
\end{aligned}
$$

and $S=\bigcup_{i=0, \ldots, m-1} S_{i}^{i+1}$. On an interface $S_{i-1}^{i}$, we denote by $n$ the outward unit normal vector to $\Omega_{i}$ and the outward unit normal vector to $\Omega_{m}$ on $\Gamma$. We set $\Omega^{\prime}=\Omega_{0} \cup \cdots \cup \Omega_{m}$.

We introduce the following geometric condition.

Assumption 5.1. Geometric Condition $\left(\mathbf{G C}_{m}\right)$ Let $\Omega_{i, j}$ be a component of $\Omega_{i}$. We assume that either

Case $1 i=0$ or $\Omega_{i, j}$ has no interface in common with any component of $\Omega_{i-1}$, i.e. $S^{i, j}=\emptyset$.

Case $2 \Omega_{i, j}$ has an interface with only one component of $\Omega_{i-1}$.

In case 1 we further assume that there exists two disjoint open subsets $\mathcal{O}_{i, j}^{(1)}$, $\mathcal{O}_{i, j}^{(2)} \Subset \Omega_{i, j}$ and two vector fields, $\zeta_{i, j}^{(k)} \in \mathscr{C}^{1}\left(\bar{\Omega}_{i, j}, \mathbb{R}^{2}\right), k=1,2$, such that

$$
\zeta_{i, j}^{(k)}(x) \cdot n(x)>0, \quad \forall x \in S_{i, j}, \quad k=1,2,
$$




$$
\begin{gathered}
\zeta_{i, j}^{(k)}(x) \cdot n(x)>0, \quad \forall x \in \partial \mathcal{O}_{i, j}^{(k)}, \quad k=1,2, \\
\zeta_{i, j}^{(k)}(x) \neq 0, \quad \forall x \in \Omega_{i, j} \backslash \mathcal{O}_{i, j}^{(k)}, \quad i=1,2 .
\end{gathered}
$$

Let $x_{i, j}^{(k)}$ be the integral curves of $\zeta_{i, j}^{(k)}$, i.e.

$$
\left\{\begin{array}{l}
\frac{d x_{i, j}^{(k)}(t)}{d t}=\zeta_{i, j}^{(k)}\left(x_{i, j}^{(k)}(t)\right), \quad t>0, \\
x_{i, j}^{(k)}(0)=x_{0}, \quad x_{0} \in S_{i, j} .
\end{array}\right.
$$

We also assume that there exists $\mathcal{T}_{i, j}>0$ such that for all $x_{0} \in S_{i, j}$, there exists $t_{i, j}^{(k)}\left(x_{0}\right)<\mathcal{T}_{i, j}$ satisfying

$$
\begin{gathered}
x_{i, j}^{(k)}(t) \in \Omega_{i, j} \backslash \mathcal{O}_{i, j}^{(k)}, \quad \text { for } 0<t<t_{i, j}^{(k)}\left(x_{0}\right), \quad x_{0} \in S_{i, j}, \quad k=1,2, \\
x_{i, j}^{(k)}\left(t_{i, j}^{(k)}\left(x_{0}\right)\right) \in \partial \mathcal{O}_{i, j}^{(k)}, \quad \text { for } x_{0} \in S_{i, j}, \quad k=1,2 .
\end{gathered}
$$

In case 2, with an interface with say $\Omega_{i-1, k}$, we further assume that there exists a vector field $\zeta_{i, j} \in \mathscr{C}^{1}\left(\bar{\Omega}_{i, j}, \mathbb{R}^{2}\right)$, such that

$$
\begin{gathered}
\zeta_{i, j}(x) \cdot n(x)>0, \quad \forall x \in S_{i, j} \\
\zeta_{i, j}(x) \cdot n(x)>0, \quad \forall x \in S_{i-1, k}^{i, j} \\
\zeta_{i, j}(x) \neq 0, \quad \forall x \in \Omega_{i, j}
\end{gathered}
$$

Let $x_{i, j}$ be the integral curves of $\zeta_{i, j}$, i.e.

$$
\left\{\begin{array}{l}
\frac{d x_{i, j}(t)}{d t}=\zeta_{i, j}\left(x_{i, j}(t)\right), \quad t>0 \\
x_{i, j}(0)=x_{0}, \quad x_{0} \in S_{i, j}
\end{array}\right.
$$

We also assume that there exists $\mathcal{T}_{i, j}>0$ such that for all $x_{0} \in S_{i, j}$, there exists $t_{i, j}\left(x_{0}\right)<\mathcal{T}_{i, j}$ satisfying

$$
\begin{gathered}
x_{i, j}(t) \in \Omega_{i, j}, \quad \text { for } 0<t<t_{i, j}\left(x_{0}\right), \quad x_{0} \in S_{i, j}, \\
x_{i, j}\left(t_{i, j}\left(x_{0}\right)\right) \in S_{i-1, k}^{i, j}, \quad \text { for } x_{0} \in S_{i, j} .
\end{gathered}
$$

Remark 5.2. In the simple case illustrated in Figure 3 where each open set $\Omega_{i}$ is connected, then only $\Omega_{0}$ falls in the case 1 and $\Omega_{1}, \ldots, \Omega_{m}$ satisfy case 2 .

We let $A_{1}=\cup_{i, j} \Omega_{i j}$ where the union is performed over the $i, j$ such that the sets $\Omega_{i, j}$ satisfy case 1 and $A_{2}=\cup_{i, j} \Omega_{i j}$ where the union is performed over the $i, j$ such that the sets $\Omega_{i, j}$ satisfy case 2 . Figures $3-6$ illustrate cases where Assumption 5.1 is, or is not, satisfied.

We now assume

Assumption 5.3. The diffusion coefficient satisfies $c_{i}:=c_{\left.\right|_{\Omega_{i}}} \in \mathscr{C}^{1}\left(\bar{\Omega}_{i}\right), i=$ $0, \ldots, m$.

Assumption 5.4. We have $0<c_{\min } \leq c(x) \leq c_{\max }, x \in \Omega^{\prime}$. If $R=S_{i, j}^{i+1, k} \neq$ $\emptyset$, for $i=0, \ldots, m-1,1 \leq j \leq p_{i}$, and $1 \leq k \leq p_{i+1}$, then $c_{\left.i\right|_{R}} \geq c_{i+\left.1\right|_{R}}$. 


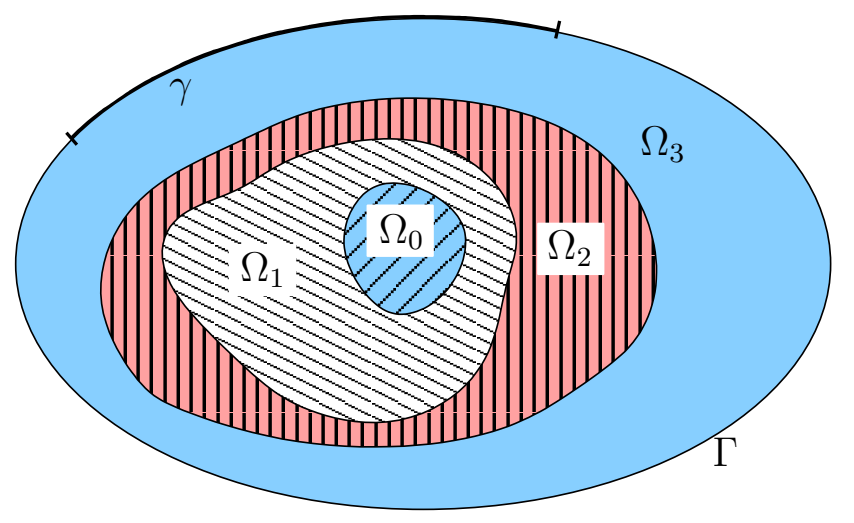

Figure 3: A geometric situation in which Assumption 5.1 is satisfied in the case $m=3$.

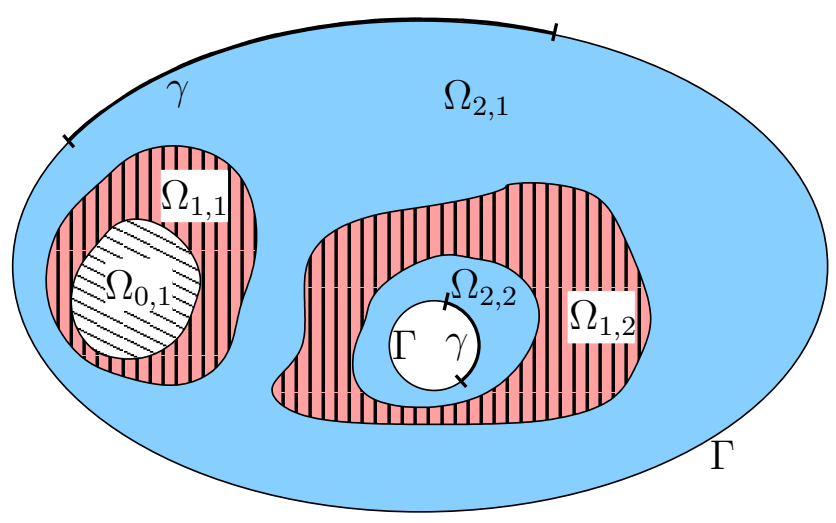

Figure 4: A geometric situation in which Assumption 5.1 is satisfied in the case $m=2$.

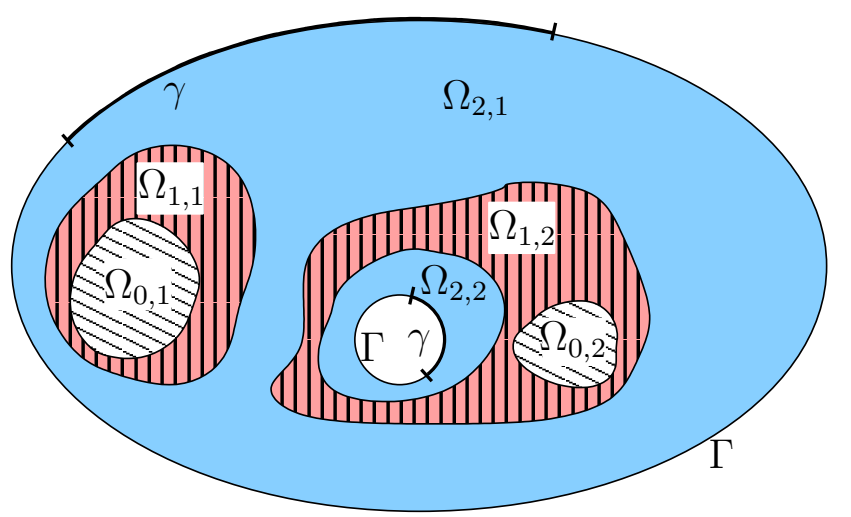

Figure 5: A geometric situation in which Assumption 5.1 is not satisfied. 


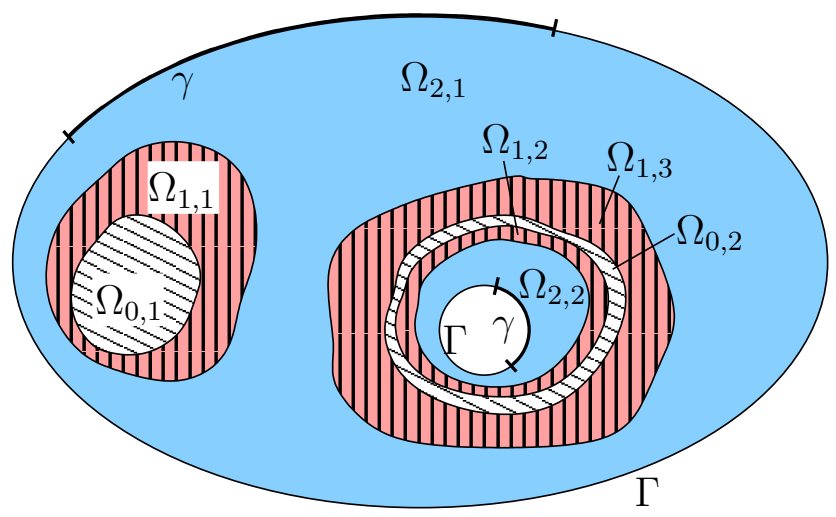

Figure 6: A geometric situation in which Assumption 5.1 is satisfied.

The geometric condition $\left(\mathrm{GC}_{m}\right)$ thus assumes that different materials are embedded in each other and the previous assumption thus states that the diffusion coefficient increases at the interfaces when one goes from the outer surface $\Gamma$ to the inner parts of the material.

We can construct weight functions for a Carleman estimate with the following lemma.

Lemma 5.5. Let the Geometric Condition $\left(G C_{m}\right)$ be satisfied and let $\gamma$ be a subset of $\Gamma=\partial \Omega$ satisfying Assumption 0.4. For the components $\Omega_{i, j}$ of $\Omega_{i}$, $i=1, \ldots, m$, satisfying case 1 in $\left(G C_{m}\right)$ we let $B_{i, j}^{(k)}$ and $\widetilde{B}_{i, j}^{(k)}, k=1,2$ be open balls such that $B_{i, j}^{(k)} \Subset \widetilde{B}_{i, j}^{(k)} \Subset \mathcal{O}_{i, j}^{(k)}, k=1,2$. Then, there exists two functions $\widetilde{\beta}^{(1)}$ and $\widetilde{\beta}^{(2)}$ in $\mathscr{C}^{0}(\bar{\Omega})$ such that

$$
\widetilde{\beta}^{(k)}(x)= \begin{cases}\widetilde{\beta}_{1}^{(k)} & \text { in } A_{1}, \\ \widetilde{\beta}_{2} & \text { in } \bar{A}_{2},\end{cases}
$$

and the functions $\widetilde{\beta}_{1}^{(1)}, \widetilde{\beta}_{1}^{(2)}$, and $\widetilde{\beta}_{2}$ satisfy the following properties:

$$
\widetilde{\beta}_{2}=0 \text { on } \Gamma \backslash \gamma, \quad \partial_{n} \widetilde{\beta}_{2}<0 \text { on } \Gamma \backslash \gamma .
$$

For $\bar{\Omega}_{i, j}$ satisfying case $2, \widetilde{\beta}_{\left.\right|_{\Omega_{i, j}}} \in \mathscr{C}^{2}\left(\bar{\Omega}_{i, j}\right), \widetilde{\beta}_{2}>0$ in $\Omega_{i, j}$, and

$$
\widetilde{\beta}_{2}=i+2 \text { on } S_{i, j}, \quad \partial_{n} \widetilde{\beta}_{2}<0 \text { on } S_{i, j} \text {, if } i<m,
$$

and

$$
\mid \nabla \widetilde{\beta}_{2}>0 \text { on } \bar{\Omega}_{i, j} .
$$

For $\bar{\Omega}_{i, j}$ satisfying case 1 , for $k=1,2,\left.\widetilde{\beta}_{1}^{(k)}\right|_{\left.\right|_{i, j}} \in \mathscr{C}^{2}\left(\bar{\Omega}_{i, j}\right), \widetilde{\beta}_{1}^{(k)}>0$ in $\Omega_{i, j}$,

$$
\begin{gathered}
\widetilde{\beta}_{1}^{(k)}=2 \text { on } S_{i, j}, \quad k=1,2, \\
\widetilde{\beta}_{1}^{(1)} \geq 2 \widetilde{\beta}_{1}^{(2)} \text { in } \widetilde{B}_{i, j}^{(2)},
\end{gathered}
$$




$$
\widetilde{\beta}_{1}^{(2)} \geq 2 \widetilde{\beta}_{1}^{(1)} \text { in } \widetilde{B}_{i, j}^{(1)}
$$

and

$$
\left|\nabla \widetilde{\beta}_{1}^{(k)}\right|>0 \text { in } \bar{\Omega}_{i, j} \backslash B_{i, j}^{(k)}, \quad k=1,2 .
$$

Finally on an (non-empty) interface $R=S_{i, j}^{i+1, k}$

$$
\left(c_{i} \partial_{n} \widetilde{\beta}^{(k)}\right)_{\left.\right|_{\Omega_{i, j}}}=\left(c_{i+1} \partial_{n} \widetilde{\beta}^{(k)}\right)_{\left.\right|_{\Omega_{i+1, k}}} \text { on } R, k=1,2 .
$$

We set $\mathcal{O}^{(k)}=\bigcup_{i, j} \mathcal{O}_{i, j}^{(k)}, k=1,2$, where the union is performed over the $i, j$ such that the sets $\Omega_{i, j}$ satisfy case 1 in Assumption 5.1.

We introduce the functions $\beta^{(k)}$ and the weight functions $\varphi^{(k)}, \eta^{(k)}, k=1,2$, following section 1. Let $g \in H^{1}\left(\left[t_{0}, T\right], H^{\frac{1}{2}}(S)\right)$. We introduce transmission condition (TC3) on the interval $\left[t_{0}, T\right]$ : for each (non-empty) interface $R=$ $S_{i, j}^{i+1, k}$, we have

$$
\begin{aligned}
& q_{\left.\right|_{\Omega_{i, j} \times\left[t_{0}, T\right]}}=q_{\left.\right|_{\Omega_{i+1, k} \times\left[t_{0}, T\right]}}, \text { on } R \\
& \left(c_{i} \partial_{n} q\right)_{\left.\right|_{\Omega_{i, j} \times\left[t_{0}, T\right]}}=\left(c_{i+1} \partial_{n} q\right)_{\left.\right|_{\Omega_{i+1, k} \times\left[t_{0}, T\right]}}+g(x, t), \text { on } R
\end{aligned}
$$

for a function $q$ which is $H^{2}$ in each open set $\Omega_{i, j}, i=0, \ldots, m, j=1, \ldots, p_{i}$. Following Section 1 we introduce

$$
\begin{aligned}
\aleph_{g}=\left\{q \in H^{1}\left(t_{0}, T, H_{0}^{1}(\Omega)\right) ;\right. & q_{\left.\right|_{\left(t_{0}, T\right) \times \Omega_{i}}} \in L^{2}\left(t_{0}, T, H^{2}\left(\Omega_{i}\right)\right), i=0,1, \\
& \left.q_{\left.\right|_{\Sigma}}=0 \text { and } q \text { satisfies (TC3) a.e. w.r.t. } t\right\} .
\end{aligned}
$$

We then obtain

Theorem 5.6. Let $\gamma$ be a subset of the boundary $\Gamma$ of an open set $\Omega$ of $\mathbb{R}^{n}$ that satisfies Condition $\left(G C_{m}\right)$, and $\gamma$ satisfies Assumption 0.4. Assume further that there exists $\Delta>0$ such that, for each (non-empty) interface $R=S_{i, j}^{i+1, k}$, we have $c_{\left.i\right|_{R}}-c_{i+\left.1\right|_{R}} \geq \Delta>0$. Let $g \in H^{1}\left(t_{0}, T, H^{\frac{1}{2}}(S)\right)$. There exists $\lambda_{1}=$ $\lambda_{1}\left(\Omega, \gamma, \mathcal{O}^{(1)}, \mathcal{O}^{(2)}, c_{\min }, c_{\max }, \Delta\right)>0, s_{1}=s_{1}\left(\lambda_{1}\right)>0$ and a positive constant $C=C\left(\Omega, \gamma, \mathcal{O}^{(1)}, \mathcal{O}^{(2)}, c_{\min }, c_{\max }, \Delta\right)$ so that Carleman estimate (1.5) holds for $s \geq s_{1}, \lambda \geq \lambda_{1}$ and for all $q \in \aleph_{g}$.

Following the method of Section 2 we thus obtain the following stability result.

Theorem 5.7. Let $\gamma$ be a subset of the boundary $\Gamma$ of an open set $\Omega$ of $\mathbb{R}^{n}$ that satisfies Condition $\left(G C_{m}\right)$, and $\gamma$ satisfies Assumption 0.4. We assume that the diffusion coefficients $c$ and $\widetilde{c}$ satisfy Assumptions 5.4 and that $c$ and $\widetilde{c}$ are piecewise constant, in the sense that $c_{\mid \Omega_{i}}$, resp. $\widetilde{c}_{\left.\right|_{\Omega_{i}}}$, are constant in each connected component of $\Omega_{i}, i=0, \ldots, m$ and that furthermore there exists $\Delta>0$ such that, for each (non-empty) interface $R=S_{i, j}^{i+1, k}$, we have $c_{\left.i\right|_{R}}-c_{i+\left.1\right|_{R}} \geq$ $\Delta>0$. Let $y_{0}, \widetilde{y}_{0}$ in $L^{2}(\Omega)$ and let $y, \widetilde{y}$ be solutions to (2.1)-(2.2) satisfying Assumption 2.1. Then there exists a constant $C$

$$
C=C\left(\Omega, T, t_{0}, \gamma, S, \mathcal{O}^{(1)}, \mathcal{O}^{(2)}, M, K, r, c_{\min }, c_{\max }, \Delta\right)
$$

such that

$$
|c-\widetilde{c}|_{L^{\infty}(\Omega)}^{2} \leq C\left|\partial_{n}\left(\partial_{t} y-\partial_{t} \widetilde{y}\right)\right|_{L^{2}((0, T) \times \gamma)}^{2}+C\left|\Delta y\left(T^{\prime}, .\right)-\Delta \widetilde{y}\left(T^{\prime}, .\right)\right|_{L^{2}\left(\Omega^{\prime}\right)}^{2} .
$$




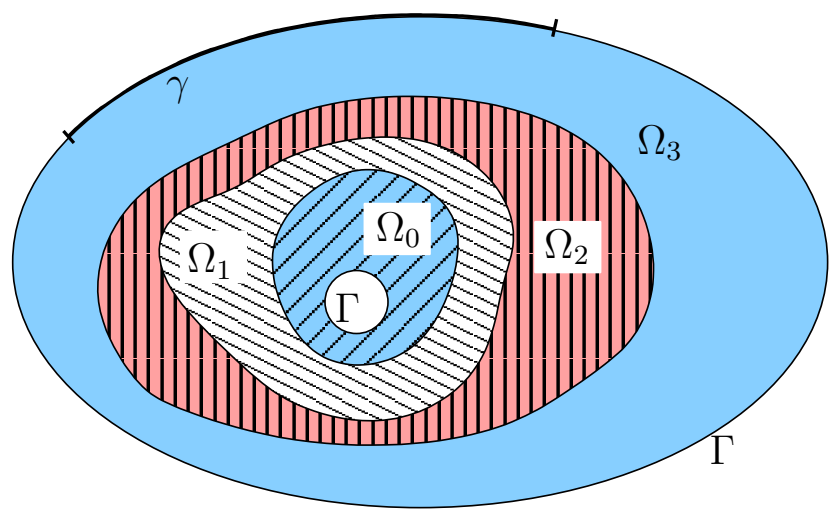

Figure 7: A geometrical situation for which only on set of weight functions is need to obtain a Carleman estimate.

Similarly, following Section 4 we obtain

Theorem 5.8. Under the hypothesis of Theorem 5.7 in addition to Assumption 4.1 there exists a constant $C>0$

$$
C=C\left(\Omega, T, t_{0}, \gamma, S, \mathcal{O}^{(1)}, \mathcal{O}^{(2)}, M, K, r, c_{\min }, c_{\max }, \Delta\right)
$$

such that

$$
\begin{aligned}
& \quad\left|y_{0}-\widetilde{y}_{0}\right|_{L^{2}(\Omega)} \leq C / \ln \left(\left|(y-\widetilde{y})\left(T^{\prime}\right)\right|_{H^{2}\left(\Omega^{\prime}\right)}+\left|\partial_{n}\left(\partial_{t} y-\partial_{t} \widetilde{y}\right)\right|_{L^{2}((0, T) \times \gamma)}^{2}\right) \\
& \text { for }\left|(y-\widetilde{y})\left(T^{\prime}\right)\right|_{H^{2}\left(\Omega^{\prime}\right)}+\left|\partial_{n}\left(\partial_{t} y-\partial_{t} \widetilde{y}\right)\right|_{L^{2}((0, T) \times \gamma)}^{2}<1 .
\end{aligned}
$$

Remark 5.9. In the generalization proposed here we assume some monotonicity for the diffusion coefficient $c$ at the inner interfaces. Note that if some of these assumptions are not satisfied we may still obtain stability results by introducing additional measurements in some $\omega \times\left(t_{0}, T\right)$ for $\omega \subset \Omega$. Each geometrical configuration requires the derivation of a particular Carleman estimate to apply the method exposed here.

Remark 5.10. Here we have assumed

$$
V_{0} \Subset V_{1} \Subset \cdots \Subset V_{m}=\Omega .
$$

Observe that this is not true in the case illustrated in Figure 7 . then $\partial_{\Omega}$ contains a component of $\Gamma$. In such a case we can however obtain a Carleman estimate by using a single set of weight functions $\eta$ and $\varphi$ instead of two (see (1.4) and (1.5)). Stability results follow similarly in such a geometrical configuration.

\section{A Basic regularity properties}

Let $\mathcal{A}$ be formally defined by $-\nabla \cdot(c \nabla()$.$) on L^{2}(\Omega)$. The diffusion coefficient $c$ is first assumed to be in $L^{\infty}(\Omega)$ and such that $c(x) \geq \alpha>0$ for all $x \in \Omega$. We 
denote by $A$ the unbounded operator with domain

$$
\mathscr{D}_{A}=\left\{u \in H_{0}^{1}(\Omega) ; \nabla \cdot(c \nabla u) \in L^{2}(\Omega)\right\} .
$$

defined by $A(u)=-\nabla \cdot(c \nabla(u))$ for $u \in \mathscr{D}_{A}$.

Lemma A.1. The operator $A$ is maximal accretive and self-adjoint on $L^{2}(\Omega)$.

Proof. Let $u \in \mathscr{D}_{A}$. We have

$$
(A u, u)_{L^{2}(\Omega)}=-\int_{\Omega} \nabla \cdot(c \nabla u) u d x=\int_{\Omega} c \nabla u \cdot \nabla u d x \geq 0
$$

since $u \in H_{0}^{1}(\Omega)$. Hence $A$ is accretive. To prove that $A$ is maximal accretive on $L^{2}(\Omega)$ we have to prove that $R(I+A)=L^{2}(\Omega)$. Let $f \in L^{2}(\Omega)$. We consider the elliptic problem

$$
\left\{\begin{array}{l}
-\nabla \cdot(c \nabla u)+u=f, \\
u_{\left.\right|_{\partial \Omega}}=0 .
\end{array}\right.
$$

We seek a weak solution, i.e. a function $u$ satisfying,

$$
\int_{\Omega} c \nabla u \cdot \nabla \varphi d x+\int_{\Omega} u \varphi d x=\int_{\Omega} f \varphi d x, \quad \forall \varphi \in H_{0}^{1}(\Omega) .
$$

We define the bilinear form $a(u, v)=\int_{\Omega} c \nabla u \cdot \nabla n d x+\int_{\Omega} u v d x$. Observe that $a$ is continuous and coercive since $c \geq \alpha>0$. The Lax-Milgram theorem thus yields a unique solution $u$ to (A.1) which is in $H_{0}^{1}(\Omega)$. Observe from (A.1) that for all $\varphi \in H_{0}^{1}(\Omega)$ we have

$$
\left|\int_{\Omega} c \nabla u \cdot \nabla \varphi d x\right| \leq C|\varphi|_{L^{2}(\Omega)} .
$$

This exactly means that $\nabla \cdot(c \nabla u) \in L^{2}(\Omega)$. The solution $u$ to (A.1) is thus in $\mathscr{D}_{A}$. Therefore, we obtain $A u+u=f$ in $L^{2}(\Omega)$ (use Corollary 2.1 in [11]). Hence $R(I+A)=L^{2}(\Omega)$.

Now, $A$ is symmetric which implies that it is self-adjoint [5, Proposition VII.6].

Proposition A.2. Let $u_{0} \in L^{2}(\Omega)$. There exists a unique $u$ such that

$$
\left.\left.\left.\left.u \in \mathscr{C}\left([0, T] ; L^{2}(\Omega)\right) \cap \mathscr{C}^{1}(] 0, T\right] ; L^{2}(\Omega)\right) \cap \mathscr{C}(] 0, T\right] ; \mathscr{D}_{A}\right),
$$

and

$$
\left\{\begin{array}{l}
\left.\left.\partial_{t} u-\nabla \cdot(c \nabla(u))=0, \quad t \in\right] 0, T\right] \\
u(0)=u_{0} .
\end{array}\right.
$$

for $T>0$. ( $T$ can be chosen to be $\infty)$. Furthermore $u \in L^{2}\left(0, T ; H_{0}^{1}(\Omega)\right)$ and $\left.\left.u \in \mathscr{C}^{k}(] 0, T\right] ; \mathscr{D}_{A^{l}}\right)$ for all $k, l \in \mathbb{N}$.

If $u_{0} \in \mathscr{D}_{A}$ then

$$
u \in \mathscr{C}^{1}\left([0, T] ; L^{2}(\Omega)\right) \cap \mathscr{C}\left([0, T] ; \mathscr{D}_{A}\right) .
$$


Proof. With Lemma A.1, we apply the Hille-Yoshida theorem for a self-adjoint operator on a Hilbert space (see e.g. [5]).

In the case $u_{0} \in \mathscr{D}_{A}$ then $\partial_{t} u(0)=-A u_{0} \in L^{2}(\Omega)[21] . \partial_{t} u$ is thus solution to (A.2) with $-A u_{0}$ for initial condition.

Proposition A.3. The semigroup $S(t)$ generated by the unbounded operator $A$ on $L^{2}(\Omega)$ is analytic.

Proof. From Theorem VII.7 in [5] and Lemma A.1 above, we obtain that $A \circ S(t)$ is bounded if $t>0$ and $\|A \circ S(t)\| \leq \frac{1}{t}$. Since 0 is in the resolvent set of $A$, we can apply Theorem 2.5.2 in [21] which yields the result.

We have obtained so far that if $u$ is the solution to (A.2) with an initial condition in $L^{2}(\Omega)$ then, $u(t,.) \in H_{0}^{1}(\Omega)$ and $\nabla \cdot(c \nabla(u)) \in L^{2}(\Omega)$, if $t>0$. If the diffusion coefficient $c$ is piecewise $\mathscr{C}^{1}$ and discontinuous across some $\mathscr{C}^{2}$ interface $S$ we can then assert that $c \partial_{n} u_{\left.\right|_{S}}$ is well defined as an element of $H^{-\frac{1}{2}}(S)$ [11, Theorem 2.2], where $\partial_{n} u_{\left.\right|_{S}}=(\nabla u)_{\left.\right|_{S}} \cdot n$ with $n$ denoting the normal unit vector to $S$.

We now give further regularity properties when placed in the geometrical situation studied in this paper. Let $\Omega \subset \mathbb{R}^{n}$ be a bounded connected open set. The set $\bar{\Omega}$ is assumed to be a $\mathscr{C}^{2}$ submanifold with boundary in $\mathbb{R}^{n}$. Let $\Omega_{0}$ and $\Omega_{1}$ be two non-empty open subsets of $\Omega$ such that

$$
\Omega_{0} \Subset \Omega \text {, and } \Omega_{1}=\Omega \backslash \bar{\Omega}_{0} .
$$

We denote $S=\bar{\Omega}_{0} \cap \bar{\Omega}_{1}$ the interface, which is assumed to be $\mathscr{C}^{2}$ and we denote $n$ the outward unit normal to $\Omega_{1}$ on $S$ and also the outward unit normal to $\Omega$ on $\Gamma$. Let $S_{0}$ (resp. $S_{1}$ ) be the side of the interface $S$ corresponding to the positive (resp. negative) direction of the normal $n$.

Proposition A.4. Let the diffusion coefficient, $c$, be such that $c_{\left.\right|_{\Omega_{i}}} \in \mathscr{C}^{1}\left(\bar{\Omega}_{i}\right)$, $i=0,1$. If $p \in \mathscr{D}_{A}$ then $p_{\left.\right|_{\Omega_{i}}} \in H^{2}\left(\Omega_{i}\right), i=0,1$. Furthermore $\left|p_{\left.\right|_{\Omega_{i}}}\right|_{H^{2}\left(\Omega_{i}\right)} \leq$ $C|\nabla \cdot(c \nabla p)|_{L^{2}(\Omega)}$.

We introduce some notations to be used in the proof of the proposition. Let us write $y \in \mathbb{R}^{n}$ in the form $y=\left(y^{\prime}, y_{n}\right)$ with $y^{\prime} \in \mathbb{R}^{n-1}$ and $y_{n}$ and define

$$
\begin{aligned}
\mathbb{R}_{+}^{n} & =\left\{y=\left(y^{\prime}, y_{n}\right) ; y_{n}>0\right\}, \\
\mathbb{R}_{-}^{n} & =\left\{y=\left(y^{\prime}, y_{n}\right) ; y_{n}<0\right\}, \\
V & =\left\{y=\left(y^{\prime}, y_{n}\right) ;\left|y^{\prime}\right|<1 \text { and }\left|y_{n}\right|<1\right\}, \\
V_{+} & =V \cap \mathbb{R}_{+}^{n}, \\
V_{-} & =V \cap \mathbb{R}_{-}^{n}, \\
V_{0} & =\left\{y=\left(y^{\prime}, 0\right) ; y \in V\right\} .
\end{aligned}
$$

Proof. The proof is along the line of that of Theorem IX.25 in [5] and makes use of Nirenberg's translation method. We set $f:=-\nabla \cdot(c \nabla p) \in L^{2}(\Omega)$. We cover $\Omega$ with a finite number of sufficiently small open balls, $\mathcal{B}_{j}, j=1, \ldots, N$ such that either 1) $\left.\left.\mathcal{B}_{j} \Subset \Omega_{0}, 2\right) \mathcal{B}_{j} \Subset \Omega_{1}, 3\right) \Omega_{0} \cap \mathcal{B}_{j}=\emptyset$ and part of $\Gamma=\partial \Omega$ is in $\mathcal{B}_{j}$, or 4) $\Omega_{i} \cap \mathcal{B}_{j} \neq \emptyset, i=0,1$, and part of $S$ is in $\mathcal{B}_{j}$. Let $\theta_{j}, j=1, \ldots, N$ be a $\mathscr{C}^{2}$ partition of unity subordinated to the covering $\mathcal{B}_{j}, j=1, \ldots, N$. 
Lemma A.5. The open balls $\mathcal{B}_{i}$ and the functions $\theta_{i}$ can be chosen such that $\partial_{n} \theta_{\left.i\right|_{S}}=0, i=1, \ldots, N$

Proof. In a small neighborhood of $S$ we can extend the unit normal vector $n$ to $S$ into a $\mathscr{C}^{2}$ vector field $v$. In an even smaller neighborhood $U$ of $S$ we can assume that $v$ is such that $|v| \geq a>0$. If we integrate this vector field we find that there is $\epsilon>0$ such that the flow $\chi_{\sigma}$ of this $\mathscr{C}^{2}$ vector field over the interval ] $-\epsilon, \epsilon[$ is confined in $U$, since $S$ is compact. For $y \in S$, the orientation of the unit normal (see above) is such that $\chi_{\sigma}(y) \in \Omega_{1}$ for $\left.\sigma \in\right]-\epsilon, 0\left[, \chi_{0}(y)=y\right.$ and $\chi_{\sigma}(y) \in \Omega_{0}$ for $\left.\sigma \in\right] 0, \epsilon[$. Set now

$$
W=\left\{\chi_{\sigma}(y) ; y \in S \text { and } \sigma \in\right]-\epsilon, \epsilon[\}
$$

which is an open neighborhood of $S$. Note that if $x$ in $W$ then there exists a unique $y \in S$ and a unique $\sigma \in]-\epsilon, \epsilon\left[\right.$ such that $x=\chi_{\sigma}(y)$.

Define $\delta=\operatorname{dist}(\mathrm{S}, \Omega \backslash \mathrm{W})$ which is positive. Cover $\overline{\Omega \backslash W}$ with a finite number of open balls of diameter $\delta / 2$, say $\mathcal{B}_{1}, \ldots, \mathcal{B}_{k}$. Thus $\mathcal{B}_{1}, \ldots, \mathcal{B}_{k}, W$ is an open covering of $\bar{\Omega}$. Note that there exists a neighborhood of $S$, contained in $W$, which does not intersect the open balls $\mathcal{B}_{1}, \ldots, \mathcal{B}_{k}$. Let $\theta_{1}, \ldots, \theta_{k}, \theta$ be a partition of unity subordinated to this covering:

$$
\begin{aligned}
\theta_{i} \in \mathscr{C}_{c}^{\infty}\left(\mathcal{B}_{i}\right), i & =1, \ldots, k, \theta \in \mathscr{C}_{c}^{\infty}(W), \\
\left(\theta+\sum_{1 \leq i \leq k} \theta_{i}\right)_{\left.\right|_{\bar{\Omega}}} & =1 .
\end{aligned}
$$

Observe that $\theta$ is equal to 1 in a neighborhood of $S$.

Let $\mathcal{C}_{j}, j=1, \ldots, r$, be open balls that form an open covering of $S$ in $\Omega$ and set $\widetilde{\mathcal{C}}_{j}=S \cap \mathcal{C}_{j}, j=1, \ldots, r$, which is an open covering of $S$ (for the topology induced on $S$ ). Let $\widetilde{\psi}_{j}, j=1, \ldots, r$, be a partition of unity subordinated to this latter covering and define now

$$
\begin{aligned}
\psi_{j}(x)=\widetilde{\psi}_{j}(y) \theta(x), x \in W, j= & 1, \ldots, r, \\
& \text { where } \left.x=\chi_{\sigma}(y), \text { with } y \in S, \sigma \in\right]-\epsilon, \epsilon[.
\end{aligned}
$$

Note that $\sum \psi_{j}=\theta$. Furthermore, $\partial_{n} \psi_{j_{S}}=\widetilde{\psi}_{j} \partial_{n} \theta_{\left.\right|_{S}}=0$. Let $N=k+r$. The functions $\theta_{1}, \ldots, \theta_{k}, \psi_{1}, \ldots, \psi_{r}$ from a partition of unity that satisfies the required property.

Continuation of the proof of Proposition A.4. We shall prove that for all $j=$ $1, \ldots, N$ the function $\theta_{j} p_{\Omega_{\Omega_{i}}}$ is in $H^{2}\left(\Omega_{i}\right), i=0,1$. This yields the result. The cases 1,2 , and 3 described above follow from the proof of Theorem IX.25 in [5]. We thus focus our attention to case 4 , i.e., when the considered ball $\mathcal{B}_{i}$ contains part of the interface $S$. For simplicity we shall write $\theta, \mathcal{B}$ instead of $\theta_{i}$ and $\mathcal{B}_{i}$.

Let $v=\theta p$. Observe that $c \nabla v=c(\nabla \theta) p+c \theta(\nabla p)$ and that, for $\psi \in H_{0}^{1}(\mathcal{B})$,

$$
\begin{aligned}
\int_{\mathcal{B}} c \nabla v \nabla \psi d x=\int_{\mathcal{B}} c(\nabla \theta) p \nabla \psi d x+\int_{\mathcal{B}} c(\nabla p) \theta \nabla \psi d x \\
=-\sum_{i=0,1} \int_{\mathcal{B} \cap \Omega_{i}} \nabla(c(\nabla \theta) p) \psi d x+\int_{\mathcal{B}} c \nabla p \nabla(\theta \psi) d x-\int_{\mathcal{B}} c(\nabla p \nabla \theta) \psi d x,
\end{aligned}
$$


since $\theta \nabla \psi=\nabla(\theta \psi)-(\nabla \theta) \psi$. The integration by parts is justified since $c \nabla \theta_{\left.\right|_{\Omega_{i}}} \in$ $\mathscr{C}^{1}\left(\overline{\Omega_{i} \cap \mathcal{B}}\right), i=0,1, p \in H^{1}(\mathcal{B})$ and $c \partial_{n} \theta_{\mid S}=0$. This finally yields

$$
\int_{\mathcal{B}} c \nabla v \nabla \psi d x=\int_{\mathcal{B}} g \psi d x
$$

where $g=-\nabla(c \nabla \theta) p+\theta f-2 c(\nabla p \nabla \theta)$ on $\Omega_{i}, i=0,1$. We have $g \in L^{2}(\mathcal{B})$ since $(c \nabla \theta)_{\left.\right|_{\Omega_{i}}}$ is in $\mathscr{C}^{1}\left(\overline{\Omega_{i} \cap \mathcal{B}}\right), i=0,1$. Observe that $|g|_{L^{2}(\mathcal{B})} \leq C|f|_{L^{2}(\Omega)}$ since $|p|_{H_{0}^{1}(\Omega)} \leq C|f|_{L^{2}(\Omega)}$.

Because of the regularity imposed on the interface $S$, there exists a $\mathscr{C}^{2}$ diffeomorphism $J: \mathcal{B} \rightarrow V$ such that $J\left(\Omega_{0} \cap \mathcal{B}\right)=V_{-}, J\left(\Omega_{1} \cap \mathcal{B}\right)=V_{+}$, and $J(S \cap \mathcal{B})=V_{0}$. Let $H=J^{-1}$. (In fact, note that in the proof of Lemma A.5 the neighborhoods $\mathcal{C}_{j}, j=1, \ldots, r$ and $\epsilon>0$ can be chosen sufficiently small so that such a diffeomorphism exists.)

Let $w=v \circ H$. Then we can prove that $w$ satisfies a problem of the form

$$
\sum_{k, l} \int_{V} \underline{c}_{k l} \partial_{x_{k}} w \partial_{x_{l}} \psi d x=\int_{V} \underline{g} \psi d x, \quad \psi \in H_{0}^{1}(V)
$$

where $\underline{c}$ satisfies the uniform ellipticity condition $\sum_{k, l} \underline{c}_{k l} \xi_{k} \xi_{l} \geq \underline{\alpha}|\xi|^{2}$ with $\underline{\alpha}>$ 0 , and $g \in L^{2}(V)$ (adapt Lemma IX.8 in [5]). The functions $\underline{c}_{k l}$ are piecewise $\mathscr{C}^{1}$ with a discontinuity across the interface $V_{0}$. Note that $w \in H_{0}^{1}(V)$ with its support finitely away from $\partial V$.

Let $h$ be parallel to $V_{0}$. Define $D_{h}$ by $D_{h}(\rho)=(\rho(x+h)-\rho(x)) /|h|$. Observe that $D_{-h}\left(D_{h} w\right) \in H_{0}^{1}(V)$ for $|h|$ sufficiently small and set $\psi=D_{-h}\left(D_{h} w\right)$. This yields

$$
\begin{aligned}
\sum_{k, l} \int_{V} D_{h}\left(\underline{c}_{k l} \partial_{x_{k}} w\right) \partial_{x_{l}}\left(D_{h} w\right) d x=\sum_{k, l} \int_{V}\left[\underline{c}_{k l}(y+h) D_{h}\left(\partial_{x_{k}} w\right)\right. \\
\left.+\left(D_{h} c_{k l}\right) \partial_{x_{k}} w\right] \partial_{x_{l}}\left(D_{h} w\right) d x=\int_{V} \underline{g} D_{-h}\left(D_{h} w\right) d x
\end{aligned}
$$

We Note that

$$
\left|\int_{V}\left(D_{h} c_{k l}\right) \partial_{x_{k}} w \partial_{x_{l}}\left(D_{h} w\right) d x\right| \leq C|w|_{H^{1}(V)}\left|\nabla\left(D_{h} w\right)\right|_{L^{2}(V)}, \quad 1 \leq k, l \leq n,
$$

and

$$
\int_{V} \underline{g} D_{-h}\left(D_{h} w\right) d x \leq|\underline{g}|_{L^{2}(V)}\left|D_{-h}\left(D_{h} w\right)\right|_{L^{2}(V)} \leq|\underline{g}|_{L^{2}(V)}\left|\nabla\left(D_{h} w\right)\right|_{L^{2}(V)}
$$

(Recall that if $\rho \in H_{0}^{1}(V)$ with its support finitely away from the boundary $\partial V$ then $\left|D_{h}(\rho)\right|_{L^{2}(V)} \leq|\nabla \rho|_{L^{2}(V)}$ for $|h|$ sufficiently small [5, Proposition IX.3].)

From (A.4) we find

$$
\underline{\alpha}\left|\nabla\left(D_{h} w\right)\right|_{L^{2}(V)}^{2} \leq C\left(|w|_{H^{1}(V)}+|\underline{g}|_{L^{2}(V)}\right)\left|\nabla\left(D_{h} w\right)\right|_{L^{2}(V)} .
$$


With $\psi=w$ we find $|\nabla w|_{L^{2}(V)} \leq C|\underline{g}|_{L^{2}(V)}$ which yields $\left|\nabla\left(D_{h} w\right)\right|_{L^{2}(V)} \leq$ $C|\underline{g}|_{L^{2}(V)}$. Thus for $\psi \in H_{0}^{1}(V)$,

$$
\begin{array}{r}
\left|\int_{V}\left(\partial_{x_{l}} w\right)\left(D_{-h} \psi\right) d x\right|=\left|\int_{V}\left(D_{h} w\right)\left(\partial_{x_{l}} \psi\right) d x\right|=\left|\int_{V} \partial_{x_{l}}\left(D_{h} w\right) \psi d x\right| \\
\leq C|\underline{g}|_{L^{2}(V)}|\psi|_{L^{2}(V)} .
\end{array}
$$

Letting $|h|$ go to zero we obtain

$$
\left|\int_{V} \partial_{x_{k}} w \partial_{x_{l}} \psi d x\right| \leq C|\underline{g}|_{L^{2}(V)}|\psi|_{L^{2}(V)}, \quad(k, l) \neq(n, n),
$$

for all $\psi \in H_{0}^{1}(V)$. Hence $\partial_{x_{k}} \partial_{x_{l}} w \in L^{2}(V)$ if $(k, l) \neq(n, n)$. Let us now choose $\psi \in H_{0}^{1}\left(V_{+}\right)$in (A.3). We obtain that in $V_{+}$

$$
\partial_{x_{n}}^{2} w=-\frac{1}{\underline{c}_{n n}}\left(f+\partial_{x_{k}}\left(\underline{c}_{k l} \partial_{x_{l}} w\right)+\left(\partial_{x_{n}} \underline{c}_{n n}\right) \partial_{x_{n}} w\right),
$$

since $\underline{c}_{n n} \geq \alpha>0$, which yields $\left.\partial_{x_{n}}^{2} w\right|_{V_{+}} \in L^{2}\left(V_{+}\right)$. Finally $w_{\left.\right|_{V_{+}}} \in H^{2}\left(V_{+}\right)$ and $\left|w_{\left.\right|_{V_{+}}}\right|_{H^{2}\left(V_{+}\right)} \leq C|g|_{L^{2}(V)}$. We proceed in a similar fashion to prove $w_{\left.\right|_{V_{-}}} \in$ $H^{2}\left(V_{-}\right)$and $\left|w_{\left.\right|_{-}}\right|_{H^{2}\left(V_{-}\right)} \leq C|g|_{L^{2}(V)}$.

Corollary A.6. Let the diffusion coefficient, $c$, be such that $c_{\left.\right|_{\Omega_{i}}} \in \mathscr{C}^{1}\left(\bar{\Omega}_{i}\right), i=$ 0,1 . If $u_{0} \in L^{2}(\Omega)$ then the solution $u$ to (A.2) is such that $u_{\left.\right|_{\Omega_{i}}}(t,.) \in H^{2}\left(\Omega_{i}\right)$, $i=0,1$, when $t>0$. Furthermore $\left|u_{\left.\right|_{\Omega_{i}}}(t, .)\right|_{H^{2}\left(\Omega_{i}\right)} \leq C\left|\partial_{t} u(t, .)\right|_{L^{2}(\Omega)}$.

Proof. Observe that $u \in \mathscr{D}_{A}$ by Proposition A.2 if $t>0$. Hence $f:=-\partial_{t} u=$ $-\nabla(c \nabla u) \in L^{2}(\Omega)$ if $t>0$.

Remark A.7. Observe that with the previous proposition the normal trace of $c \nabla u$ on $S$, i.e. $c \partial_{n} u_{\left.\right|_{S}}$, is now found to belong to $H^{\frac{1}{2}}(S)$.

Requiring additionnal smoothness for the diffusion coefficient, $\mathrm{c}$, in the open sets $\Omega_{i}, i=0,1$, and for the boundary $\partial_{\Omega}$ and the interface $S$ we can improve the previous result.

Proposition A.8. Let $m \in \mathbb{N}$ and let $c_{\left.\right|_{\Omega_{i}}} \in \mathscr{C}^{m+1}\left(\bar{\Omega}_{i}\right), i=0,1$, and let $S$ and $\partial \Omega$ be of class $\mathscr{C}^{m+2}$. If $p \in \mathscr{D}_{A}$ and $A(p)=f$ with $f_{\mid \Omega_{i}} \in H^{m}\left(\Omega_{i}\right)$ then $p_{\left.\right|_{\Omega_{i}}} \in H^{m+2}\left(\Omega_{i}\right), i=0,1$. Furthermore $\left|p_{\left.\right|_{\Omega_{i}}}\right|_{H^{m+2}\left(\Omega_{i}\right)} \leq C\left|f_{\left.\right|_{\Omega_{i}}}\right|_{H^{m}\left(\Omega_{i}\right)}$, $i=0,1$.

Proof. We proceed by induction. The case $m=0$ is contained in the proof of Proposition A.4. Let $m_{0}>0$. Assume the result is true for $0 \leq m \leq$ $m_{0}-1$ and assume $f_{\left.\right|_{\Omega_{i}}} \in H^{m_{0}}\left(\Omega_{i}\right), i=0,1$. Thus, $f_{\left.\right|_{\Omega_{i}}} \in H^{m_{0}-1}\left(\Omega_{i}\right)$ which yields $p_{\mid \Omega_{i}} \in H^{m_{0}+1}\left(\Omega_{i}\right)$ by induction, and $\left|p_{\mid \Omega_{i}}\right|_{H^{m_{0}+1}\left(\Omega_{i}\right)} \leq C\left|f_{\mid \Omega_{i}}\right|_{H^{m_{0}-1}\left(\Omega_{i}\right)} \leq$ $C\left|f_{\mid \Omega_{i}}\right|_{H^{m_{0}}\left(\Omega_{i}\right)}, i=0,1$.

We use the same partition of unity $\theta_{j}, j=1, \ldots, N$, as in the proof of Proposition A.4 and prove the result for $v:=\theta_{i} p$, i.e. $v_{\left.\right|_{\Omega_{i}}} \in H^{m_{0}+2}\left(\Omega_{i}\right), i=0,1$. We choose here the functions $\theta_{i}$ to be $\mathscr{C}^{2 m_{0}+2}$. We focus our attention to the 
case where $\operatorname{supp}\left(\theta_{i}\right)$ contains part of $S$ since the other cases follow from classical results. We obtain

$$
\int_{\mathcal{B}} c \nabla v \nabla \psi d x=\int_{\mathcal{B}} g \psi d x, \quad \psi \in H_{0}^{1}(\mathcal{B})
$$

where $g=-\nabla(c \nabla \theta) p+\theta f-2 c(\nabla p \nabla \theta)$ on $\Omega_{i}, i=0,1$. Observe that $g_{\left.\right|_{\Omega_{i}}} \in$ $H^{m_{0}}\left(\Omega_{i}\right)$ and $\left|g_{\Omega_{i}}\right|_{H^{m_{0}\left(\Omega_{i}\right)}} \leq\left|f_{\left.\right|_{\Omega_{i}}}\right|_{H^{m_{0}\left(\Omega_{i}\right)}}, i=0,1$.

With the notations of the proof of Proposition A.4 let $w=v \circ H$. Then $w$ satisfies

$$
\sum_{k, l} \int_{V} \underline{c}_{k l} \partial_{x_{k}} w \partial_{x_{l}} \psi d x=\int_{V} \underline{g} \psi d x, \quad \psi \in H_{0}^{1}(V)
$$

where $\underline{c}$ is uniformly elliptic and $\mathscr{C}^{m+1}$ in each $\Omega_{i}, i=0,1$, and $\underline{g}_{V_{ \pm}} \in H^{m_{0}}\left(V_{ \pm}\right)$ and

$$
\begin{aligned}
& \left|\underline{g}_{V_{+}}\right|_{H^{m_{0}}\left(V_{+}\right)} \leq\left|f_{\left.\right|_{\Omega_{1}}}\right|_{H^{m_{0}}\left(\Omega_{1}\right)}, \\
& \left|\underline{g}_{V_{V_{-}}}\right|_{H^{m_{0}}\left(V_{-}\right)} \leq\left|f_{\Omega_{\Omega_{0}}}\right|_{H^{m_{0}}\left(\Omega_{0}\right)} .
\end{aligned}
$$

As in the proof of Lemma IX.7 in [5] we can show $\partial_{x_{j}} w \in H_{0}^{1}(V)$ for $j=$ $1, \ldots, n-1$. Choosing $\psi=\partial_{x_{j}} \varphi$ with $\varphi \in \mathscr{C}_{c}^{\infty}(V)$ we obtain

$$
\sum_{k, l} \int_{V} \underline{c}_{k l} \partial_{x_{k}}\left(\partial_{x_{j}} w\right) \partial_{x_{l}} \varphi d x=\int_{V} \underline{\tilde{g}} \varphi d x, \quad \varphi \in \mathscr{C}_{c}^{\infty}(V)
$$

with $\underline{\widetilde{g}}_{\left.\right|_{V_{ \pm}}} \in H^{m_{0}-1}\left(V_{ \pm}\right)$satisfying $\left|\underline{\widetilde{g}}_{\left.\right|_{V_{ \pm}}}\right|_{H^{m_{0}-1}\left(V_{ \pm}\right)} \leq C\left|\underline{g}_{V_{ \pm}}\right|_{H^{m_{0}}\left(V_{ \pm}\right)}$. From the induction we obtain $\left(\partial_{x_{j}} w\right)_{\left.\right|_{ \pm}} \in H^{m_{0}+1}\left(V_{ \pm}\right), j=1, \ldots, n-1$ with

$$
\left|\left(\partial_{x_{j}} w\right)_{\left.\right|_{ \pm}}\right|_{H^{m_{0}+1}\left(V_{ \pm}\right)} \leq\left|\underline{g}_{V_{ \pm}}\right|_{H^{m_{0}}\left(V_{ \pm}\right)}
$$

(here, we implicitly proceed with an induction proof for the regularity of $v$ ). Since $w_{\left.\right|_{ \pm}} \in H^{m_{0}+1}\left(V_{ \pm}\right)$, to conclude we have to prove

$$
\left|\int_{V_{ \pm}} \partial_{x_{n}} w \partial^{\alpha} \psi d x\right| \leq C\left|\underline{g}_{V_{ \pm}}\right| H_{H_{0}\left(V_{ \pm}\right)}|\psi|_{L^{2}\left(V_{ \pm}\right)}, \quad \psi \in \mathscr{C}_{c}^{\infty}\left(V_{ \pm}\right),|\alpha|=m_{0}+1,
$$

where $\alpha$ is a multi-index $\alpha=\left(\alpha_{1}, \ldots, \alpha_{n}\right)$. We first treat the case $\alpha_{j} \neq 0$ for some $j$ in $\{1, \ldots, n-1\}$, say $\alpha_{1} \neq 0$ then

$$
\int_{V_{ \pm}} \partial_{x_{n}} w \partial^{\alpha} \psi d x=\int_{V_{ \pm}} \partial_{x_{1}} w \partial^{\alpha^{\prime}} \psi d x, \quad \text { with } \alpha^{\prime}=\left(\alpha_{1}-1, \alpha_{2}, \ldots, \alpha_{n-1}, 1\right)
$$

since $\psi \in \mathscr{C}_{c}^{\infty}\left(V_{ \pm}\right)$and we obtain estimate (A.6) since $\partial_{x_{1}} w_{\left.\right|_{V_{ \pm}}} \in H^{m_{0}+1}\left(V_{ \pm}\right)$ and $\mid \alpha^{\prime}=m_{0}+1$.

We write in $V_{ \pm}$

$$
\partial_{x_{n}}^{2} w=-\frac{1}{\underline{c}_{n n}}\left(f+\partial_{x_{k}}\left(\underline{c}_{k l} \partial_{x_{l}} w\right)+\left(\partial_{x_{n}} \underline{c}_{n n}\right) \partial_{x_{n}} w\right),
$$

and find $\partial_{x_{n}}^{2} w_{\left.\right|_{V_{ \pm}}} \in H^{m_{0}}\left(V_{ \pm}\right)$which yields estimate (A.6) in the case $\alpha=$ $\left(0, \ldots, 0, m_{0}+1\right)$. 
Corollary A.9. Let $l \in \mathbb{N}$ and let $c_{\left.\right|_{\Omega_{i}}} \in \mathscr{C}^{2 l+1}\left(\Omega_{i}\right), i=0,1$, and $S$ and $\partial \Omega$ be of class $\mathscr{C}^{2 l+2}$. If $p \in \mathscr{D}_{A^{l+1}}$ then $p_{\left.\right|_{\Omega_{i}}} \in H^{2 l+2}\left(\Omega_{i}\right)$ and $\left|p_{\left.\right|_{\Omega_{i}}}\right|_{H^{2 l+2}\left(\Omega_{i}\right)} \mid \leq$ $C\left|A^{l+1}(p)\right|_{L^{2}(\Omega)}, i=0,1$.

Proof. We proceed by induction. The case $l=0$ is contained in Proposition A.4. Assume the result is true for $l \leq l_{0}-1$ and assume $p \in \mathscr{D}_{A^{l_{0}+1}}$. Thus $f:=A(p) \in$ $\mathscr{D}_{A^{l_{0}}}$ and thus $f_{\left.\right|_{\Omega_{i}}} \in H^{2 l_{0}}\left(\Omega_{i}\right)$, and $\left.\left|f_{\left.\right|_{\Omega_{i}}}\right|_{H^{2 l_{0}\left(\Omega_{i}\right)}}|\leq C| A^{l_{0}+1}(p)\right|_{L^{2}(\Omega)}, i=0,1$. Finally $p_{\left.\right|_{\Omega_{i}}} \in H^{2 l_{0}+2}\left(\Omega_{i}\right)$ and $\left.\left|p_{\left.\right|_{\Omega_{i}}}\right|_{H^{2 l_{0}+2}\left(\Omega_{i}\right)}|\leq C| A^{l_{0}+1}(p)\right|_{L^{2}(\Omega)}, i=0,1$, by Proposition A.8.

Remark A.10. Observe that the constant in the inequalities of the previous results can be chosen uniform w.r.t. $c$ for $c_{\min } \leq c \leq c_{\max }$.

Combining the results just obtained and those of Proposition A.2 we obtain

Corollary A.11. Let $m \in \mathbb{N}$ and let $c_{\left.\right|_{\Omega_{i}}} \in \mathscr{C}^{m+1}\left(\Omega_{i}\right), i=0,1$, and $S$ and $\partial \Omega$ be of class $\mathscr{C}^{m+2}$. Then if $u_{0} \in L^{2}(\Omega)$ the solution $u$ to (A.2) is such that $u_{\left.\right|_{\Omega_{i}}} \in \mathscr{C}^{k}\left((0, T], H^{m+2}\left(\Omega_{i}\right)\right), i=0,1$, for all $k \in \mathbb{N}$.

Proof. Let $k \in \mathbb{N}$ and let $l \in \mathbb{N}$ be such that $2 l \leq m \leq 2 l+2$. Since $u \in \mathscr{C}^{k}\left((0, T], \mathscr{D}_{A^{l+1}}\right)$ then $u_{\left.\right|_{\Omega_{i}}} \in \mathscr{C}^{k}\left((0, T], H^{2 l+2}\left(\Omega_{i}\right)\right)$ by Corollary A.9 since $c_{\Omega_{\Omega_{i}}} \in \mathscr{C}^{2 l+1}\left(\Omega_{i}\right)$. In the case $m=2 l$ we have obtained the sought result.

In the case $m=2 l+1$ we similarly find $A u_{\left.\right|_{\Omega_{i}}} \in \mathscr{C}^{k}\left((0, T], H^{m+1}\left(\Omega_{i}\right)\right)$. Then by Proposition A.8 we obtain the result.

Remark A.12. Let $\varepsilon>0$. With the notations of the above corollary, observe that the map

$$
\begin{aligned}
L_{\varepsilon, i}: L^{2}(\Omega) & \rightarrow \mathscr{C}^{k}\left([\varepsilon, T], H^{m+2}\left(\Omega_{i}\right)\right), \\
u_{0} & \mapsto\left(t \mapsto u_{\left.\right|_{\Omega_{i}}}(t)\right),
\end{aligned}
$$

is continuous for $i=0,1$, since [5, Theorem VII.7]

$$
\begin{aligned}
|u(t)|_{L^{2}(\Omega)} & \leq\left|u_{0}\right|_{L^{2}(\Omega)}, \\
\left|\partial_{t} u(t)\right|_{L^{2}(\Omega)} & =|\nabla \cdot(c \nabla u(t))|_{L^{2}(\Omega)} \leq\left.\left|\frac{1}{t}\right| u_{0}\right|_{L^{2}(\Omega)} .
\end{aligned}
$$

Note that the operator norm of $L_{\varepsilon, i}$ can be bounded uniformly w.r.t. $c$ for $c_{\text {min }} \leq c \leq c_{\text {max }}$.

Acknowledgement: The authors wish to thank M. Cristofol, Y. Dermenjian, and O. Poisson for numerous discussions on the subjet of this article. The authors also thank F. Boyer for helpful comments. The authors also wish to thank an anonymous reviewer for his extremely detailed comments and corrections, which improved the exposition of the article. 


\section{References}

[1] R. Adams. Sobolev Spaces. Academic Press, New York, 1975.

[2] L. Baudouin and J.-P. Puel. Uniqueness and stability in an inverse problem for the Schrödinger equation. Inverse Problems, 18:1537-1554, 2002.

[3] A. Benabdallah, Y. Dermenjian, and J. Le Rousseau. Carleman estimates for the one-dimensional heat equation with a discontinuous coefficient and applications. Comptes Rendus Mécanique, 334:582-586, 2006.

[4] A. Benabdallah, Y. Dermenjian, and J. Le Rousseau. Carleman estimates for the one-dimensional heat equation with a discontinuous coefficient and applications to controllability and an inverse problem. Preprint: LATP, Université d'Aix-Marseille I, www.cmi.univ-mrs.fr/ jlerous/publications.html, 2006.

[5] H. Brezis. Analyse fonctionnelle. Masson, Paris, 1983.

[6] R. Dautray and J.-L. Lions. Analyse mathématique et calcul numérique pour les sciences et les techniques, volume 7. Masson, Paris, 1984.

[7] A. Doubova, A. Osses, and J.-P. Puel. Exact controllability to trajectories for semilinear heat equations with discontinuous diffusion coefficients. ESAIM: COCV, 8:621-661, 2002.

[8] H. Egger, H. W. Engl, and M. V. Klibanov. Global uniqueness and hölder stability for recovering a nonlinear source term in a parabolic equation. Inverse problems, 21:271-290, 2005.

[9] E. Fernández-Cara and S. Guerrero. Global Carleman inequalities for parabolic systems and application to controllability. SIAM J. Control Optim., to appear.

[10] A. Fursikov and O. Yu. Imanuvilov. Controllability of evolution equations, volume 34. Seoul National University, Korea, 1996. Lecture notes.

[11] V. Girault and P.-A. Raviart. Finite element approximation of the NavierStokes equations. Springer-Verlag, New York, 1979.

[12] P. Grisvard. Elliptic problems in nonsmooth domains. Pitman, Boston, 1985.

[13] L. Hörmander. Linear partial differential operators. Springer-Verlag, Berlin, 1963.

[14] L. Hörmander. The analysis of linear partial differential operators, volume IV. Springer-Verlag, 1985.

[15] L. Hörmander. The analysis of linear partial differential operators, volume I. Springer-Verlag, second edition, 1990.

[16] V. Isakov. Carleman type estimates in an anisotropic case and applications. J. Diff. Eq., 105:217-238, 1993. 
[17] V. Isakov. Inverse problems for partial differential equations. SpringerVerlag, Berlin, 1998.

[18] M. V. Klibanov. Global uniqueness of a multidimensional inverse problem for a nonlinear parabolic equation by a Carleman estimate. Inverse problems, 20:1003-1032, 2004.

[19] O. Yu. Imanuvilov and M. Yamamoto. Lipschitz stability in inverse problems by Carleman estimate. Inverse problems, 14:1229-1245, 1998.

[20] L. E. Payne. Improperly posed problems in partial differential equations. SIAM, Philadelphia, 1975.

[21] A. Pazy. Semigroups of linear operators and applications to partial differential equations. Springer-Verlag, New York, 1983.

[22] M. Yamamoto and J. Zou. Simultaneous reconstruction of the initial temperature and heat radiative coefficient. Inverse problems, 17:1181-1202, 2001. 